\title{
MODELS AND MINING OF ON-LINE SOCIAL NETWORKS
}

by

Yanhua Tian, B.Sc. Tianjin University, 1999

\author{
A thesis \\ presented to Ryerson University \\ in partial fulfillment of the \\ requirements for the degree of \\ Master of Science \\ in the Program of \\ Applied Mathematics
}

Toronto, Ontario, Canada, 2011

(C) Copyright by Yanhua Tian 2011 
I hereby declare that I am the sole author of this thesis or dissertation. I authorize Ryerson University to lend this thesis or dissertation to other institutions or individuals for the purpose of scholarly research.

Signature:

I further authorize Ryerson University to reproduce this thesis or dissertation by photocopying or by other means, in total or in part, at the request of other institutions or individuals for the purpose of scholarly research.

Signature: 


\begin{abstract}
Models and Mining of On-line Social Networks

Master of Science, 2011

Yanhua Tian

Applied Mathematics, Ryerson University
\end{abstract}

Power law degree distribution, the small world property, and bad spectral expansion are three of the most important properties of On-line Social Networks (OSNs). We sampled YouTube and Wikipedia to investigate OSNs. Our simulation and computational results support the conclusion that OSNs follow a power law degree distribution, have the small world property, and bad spectral expansion.

We calculated the diameters and spectral gaps of OSNs samples, and compared these to graphs generated by the GEO-P model. Our simulation results support the Logarithmic Dimension Hypothesis, which conjectures that the dimension of OSNs is $m=\lceil\log N\rceil$.

We introduced six GEO-P-type models. We ran simulations of these GEO-P-type models, and compared the simulated graphs with real OSN data. Our simulation results suggest that, except for the 
GEO-P(GnpDeg) model, all our models generate graphs with power law degree distributions, the small world property, and bad spectral expansion. 


\section{Acknowledgements}

First and foremost I offer my sincerest gratitude to my supervisor,

Dr. Anthony Bonato, for his encouragement, guidance and support from the initial stage of this project and giving me extraordinary academic experiences through out the work.

I extend my thanks to the Department of Mathematics at Ryerson University, and especially to Mr. Stephen Kanellis, who supplied computer support. A special thanks to Dr. Peter Danziger and Dr. Dejan Delić for being part of my thesis committee.

Finally, I would like to thank my family, especially my husband Yong, for their constant support in all aspects of my life. 



\section{Contents}

Abstract

Acknowledgements $\quad \mathrm{v}$

List of Figures ix

Chapter 1. Introduction of On-line Social Networks 1

1. Graph Theory 3

2. Overview of Thesis 14

$\begin{array}{lll}\text { Chapter 2. } & \text { Geometric models for OSNS } & 15\end{array}$

1. Introduction to the GEO-P model 15

2. Results on GEO-P 22

3. Dimension of the GEO-P model 28

$\begin{array}{lll}\text { Chapter 3. } & \text { Properties of Real OSN Data } & 31\end{array}$

1. Properties of Real OSNS 31

2. $\quad$ Properties of Graphs Generated by the GEO-P Model 36

3. Logarithmic Dimension Hypothesis 38

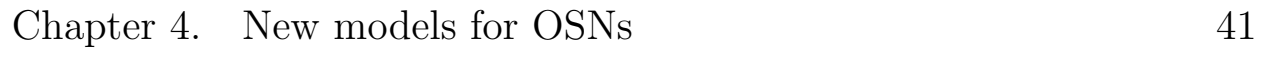


\begin{tabular}{lll}
\hline 1. & Ranking by Age & 41
\end{tabular}

2. $\quad$ Ranking by Degree 45

3. Tension Parameter 51

\begin{tabular}{lll}
\hline Chapter 5. Conclusion and Open Problems & 57
\end{tabular}

\begin{tabular}{lll}
\hline Chapter 6. & Appendix & 61
\end{tabular}

\begin{tabular}{ll}
\hline Bibliography & 131
\end{tabular} 


\section{List of Figures}

1.1 A friendship graph sampled from Wikipedia. 3

1.2 An undirected graph $G$ with 4 vertices and 5 edges. 4

1.3 The subgraph $H$ induced by $\left\{v_{1}, v_{2}, v_{3}\right\}$.

\begin{tabular}{ll}
\hline 1.4 A random graph $G\left(20, \frac{1}{2}\right)$. & 7
\end{tabular}

\begin{tabular}{ll}
\hline 1.5 Wikipedia degree distribution log-log plot. & 10
\end{tabular}

2.120 randomly chosen vertices in a 2-dimensional unit hypercube. 16

2.2 Graph generated by 20 vertices at $p=1$. 20

2.3 Graph generated by the GEO-P model, $\alpha=0.7, \beta=0.15$, and

$\begin{array}{ll}p=1 . & 21\end{array}$

3.1 YouTube sample degree distribution. 32

3.2 YouTube sample degree distribution log-log plot. 32

3.3 GEO-P degree distribution $\log -\log$ plot, $N=7115, \alpha=0.7$,

$\begin{array}{ll}\beta=0.15, p=1 . & 37\end{array}$

3.4 GEO-P degree distribution $\log$-log plot, $N=7115, \alpha=0.4$,

$\begin{array}{ll}\beta=0.5, p=1 . & 37\end{array}$ 
4.1 Degree distribution of a graph generated by the GEO-P(Age)

model.

44

4.2 Degree distribution of a graph generated by the GEO-

P(InvAge) model.

44

4.3 Degree distribution of graph generated by the GEO-P(Deg)

model.

50

4.4 Degree distribution of graph generated by the GEO-P(GnpDeg)

model.

50

4.5 Influence region of vertices 5,7 and 16 .

4.6 Degree distribution of graph generated by the GEO-P(Ten)

model, with tension parameter $h=-0.1$.

54

4.7 Degree distribution of graph generated by the GEO-P(Ten)

model, with tension parameter $h=-0.3$.

55

4.8 Degree distribution of graph generated by the GEO-P(Ten)

model, with tension parameter $h=-0.7$.

55 


\section{CHAPTER 1}

\section{Introduction of On-line Social Networks}

With the development of computer and network technology, On-line Social Networks (OSNs) have become increasingly important in human society. An OSN is an internet-based platform to communicate, network, and share information among people. Examples of OSNs include Facebook and Twitter. Compared to traditional social networks, OSNs allow users to spread information quickly and to a large audience. Facebook, one of the most popular OSNs, has now more than 500 million active users and an average user has 130 friends (see [17]).

Computed-mediated communication stretches back to the late 1970s. Usenet, the oldest computer network communications systems, was created by two Duke University graduate students, Tom Truscott and Jim Ellis (see [19, 20]). By the late 1990s, with the boom of the Internet, a new generation of internet-mediated communication services began to flourish. Today there are over 200 major active on-line social networking websites (see, for example, [14]). 
The studies of social networking go back to the 1960s. In 1967, Milgram and other researchers conducted several experiments examining the interconnectedness among human beings. Milgram and his research group proposed the theory that in human society every person can on average approximately reach another person in just six steps, which refers to the famous phrase "six degrees of separation" (see [15]). In 1998, Watts and Strogatz did further study on this topic. They defined the small world property for social networks, and introduced a random graph generation model that produces graphs with the small world property (see [21]).

From 2000 onwards, as they have become more and more popular, OSNs have attracted more attention from scientists. In 2003, Adamic et al. gave an early study of OSNs. They chose Club Nexus as a sample to investigate properties, such as distance between users and the clustering coefficient (see [1]). In 2005, in his Ph.D thesis, LibenNowell studied Livejournal and showed that this OSN follows the small world property (see [13]). In 2006, Kumar et al. studied the evolution of OSNs by studying with Flickr and Yahoo! 360 (see [12]). Moreover, Golder et al. (see [11]), Ahn et al. (see [2]), Mislove et al. (see [6]) have studied the OSNs Facebook, Myspace, Orkut, and Flickr. 


\section{Graph Theory}

Graph theory is a natural tool to model and simulate the evolution of OSNs. To present an OSN by a graph, we represent people as vertices, and friendship between people as edges. For example, we retrieved a sample of the Wikipedia OSN (see [18]) to generate its friendship network; see Figure 1.1

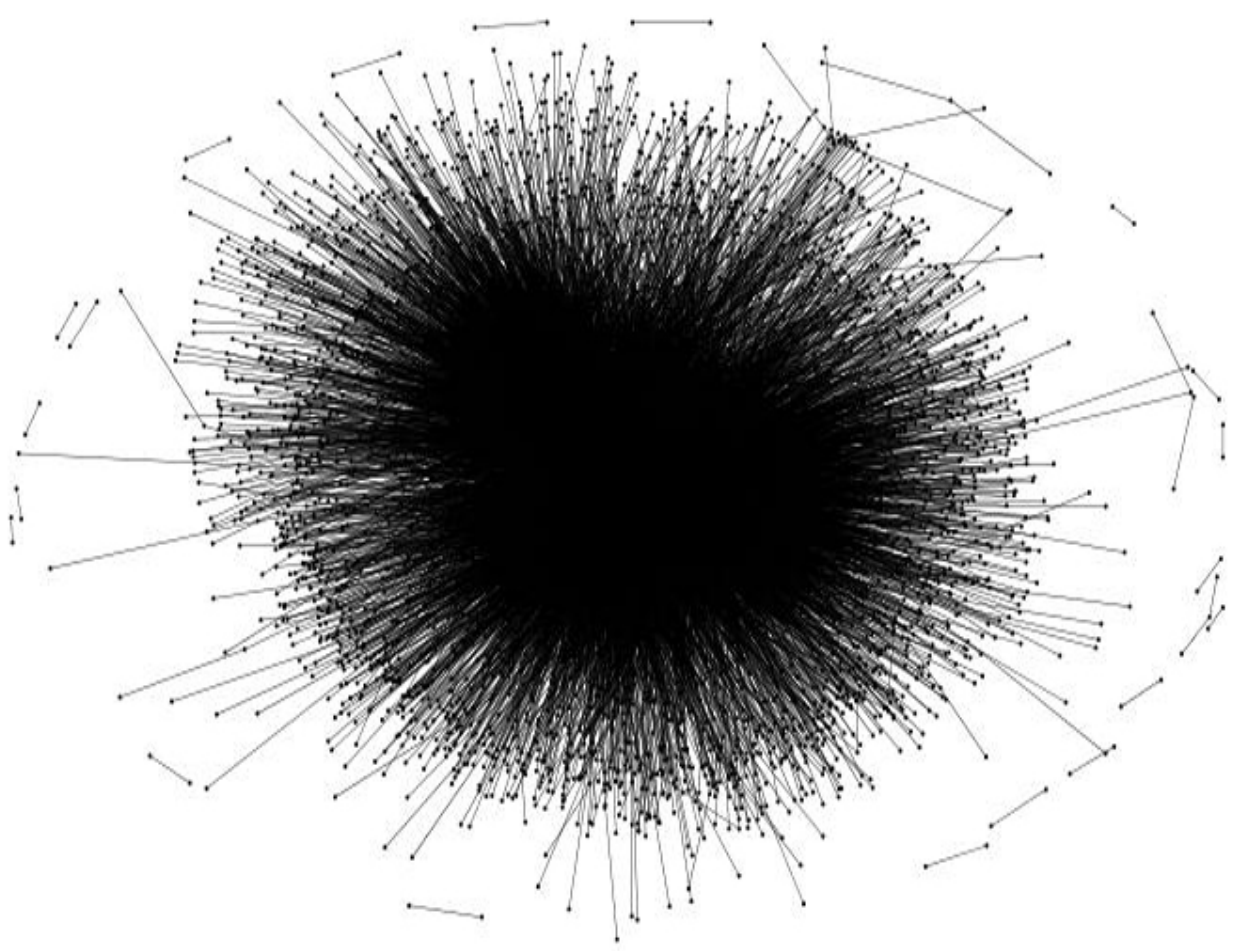

FiguRE 1.1. A friendship graph sampled from Wikipedia.

1.1. Introduction to graphs. We now give a precise definition of a graph. A graph $G$ consists of a non-empty vertex set $V(G)$, and 
an edge set $E(G)$ of unordered 2-elements sets from $V(G)$; we may also consider $E(G)$ as a binary relation on $V(G)$. A graph $G$ can be written as $G=(V(G), E(G))$, or if $G$ is clear from the context, $G=(V, E)$. In a graph $G$, elements of $V(G)$ are vertices, and the edge set $E(G)$ can be empty. We call a graph as undirected graph if relations between pairs of vertices are symmetric and edges are not directed. In this case, an edge is represented by $u v$ for vertices $u$ and $v$. In this thesis, we will mainly focus on simple graphs, which are undirected graphs without loops, and where there is at most one edge between every pair of distinct vertices.

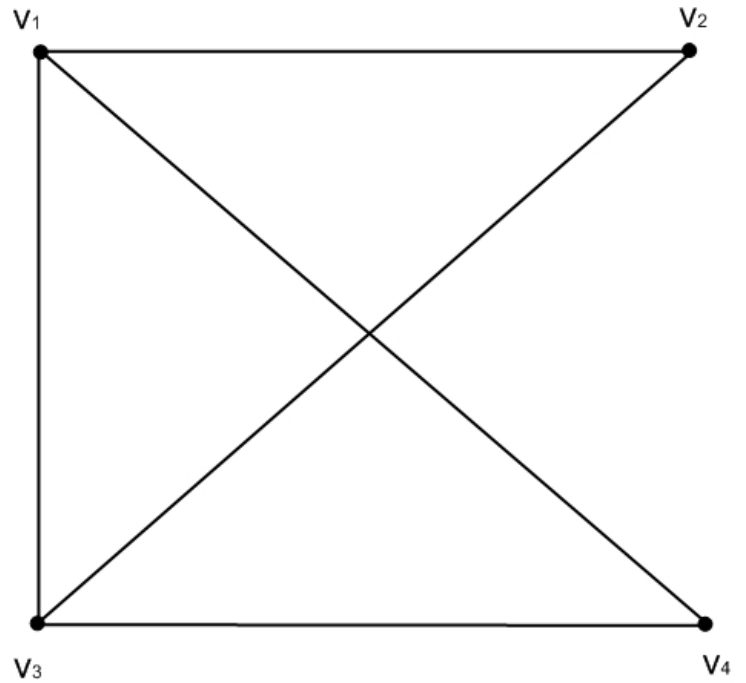

Figure 1.2. An undirected graph $G$ with 4 vertices and 5 edges. 
Figure 1.2 shows an example of an undirected graph. The vertex set and edge set of this graph $G$ are:

$$
\begin{aligned}
& V(G)=\left\{v_{1}, v_{2}, v_{3}, v_{4}\right\} \\
& E(G)=\left\{v_{1} v_{2}, v_{1} v_{3}, v_{1} v_{4}, v_{2} v_{3}, v_{3} v_{4}\right\} .
\end{aligned}
$$

The cardinality $|V(G)|$ is the order of the graph $G$. With the example of Figure 1.2, the order of the graph $G$ is 4 . We use $\operatorname{deg}_{G}(x)$ to present the degree of a given vertex $x$ in graph $G$, which is the number of edges joined to the vertex $x$. With the example of Figure 1.2 .

$$
\begin{aligned}
& \operatorname{deg}_{G}\left(v_{1}\right)=\operatorname{deg}_{G}\left(v_{3}\right)=3, \\
& \operatorname{deg}_{G}\left(v_{2}\right)=\operatorname{deg}_{G}\left(v_{4}\right)=2 .
\end{aligned}
$$

A subgraph of graph $G$ is a graph whose vertex set is a subset of $V(G)$, and whose edge set is a subset of $E(G)$. A subgraph $H$ is called an induced subgraph of graph $G$ if $V(H) \subseteq V(G)$, and for any pair of vertices $u$ and $v$ of $\mathrm{H}, u v \in E(H)$ if and only if $u v \in E(G)$. For example, Figure 1.3 shows a induced subgraph $H$ of the graph $G$ of Figure 1.2 .

We now give a formal definition of random graphs. Define a probability space on graphs of a given order $N \geq 1$ as follows. Fix a 


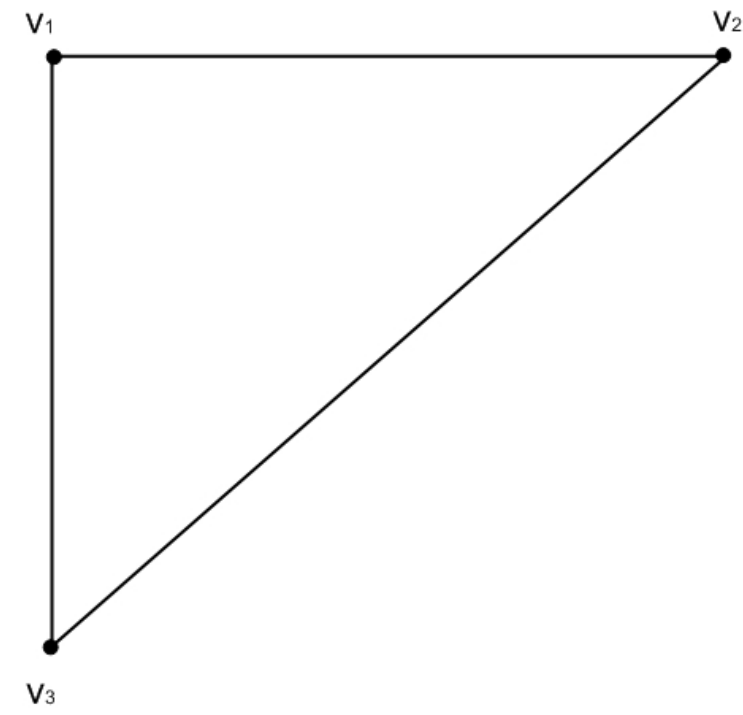

Figure 1.3. The subgraph $H$ induced by $\left\{v_{1}, v_{2}, v_{3}\right\}$.

vertex set $V$ consisting of $N$ distinct elements, usually taken as $[N]=$ $\{1,2, \ldots, N\}$, and fix $p \in[0,1]$. Define the space of random graphs of order $N$ with edge probability $p$, written $G(N, p)$ with sample space

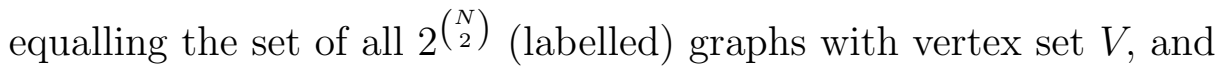

$$
\mathbb{P}(G)=p^{|E(G)|_{(1-p)}}\left(\begin{array}{c}
N \\
2
\end{array}\right)-|E(G)|,
$$

where $\mathbb{P}(A)$ is the probability that event $A$ occurs.

Informally, we may view $G(N, p)$ as the space of graphs with vertex set $V$, so that two distinct vertices are joined independently with probability $p$. Even more informally: toss a (biased) coin to determine the edges of your graph. Hence, $V$ does not change, but the number of 
edges is not fixed: it varies according to a binomial distribution with expectation $\left(\begin{array}{c}N \\ 2\end{array}\right) p$. Despite the fact that $G(N, p)$ is a space of graphs, we will abuse language and call it the random graph of order $N$ with edge probability $p$.

We will consider the cases when $p$ is fixed, and when it is a function of $N$. Graph parameters, such as diameter and average degree, become random variables in $G(N, p)$. We say that an event holds asymptotically almost surely (or a.a.s. for short) if it holds with probability tending to 1 as $N \rightarrow \infty$. Figure 1.4 shows a random graph with 20 vertices, and with edge drawn randomly with probability of $\frac{1}{2}$.

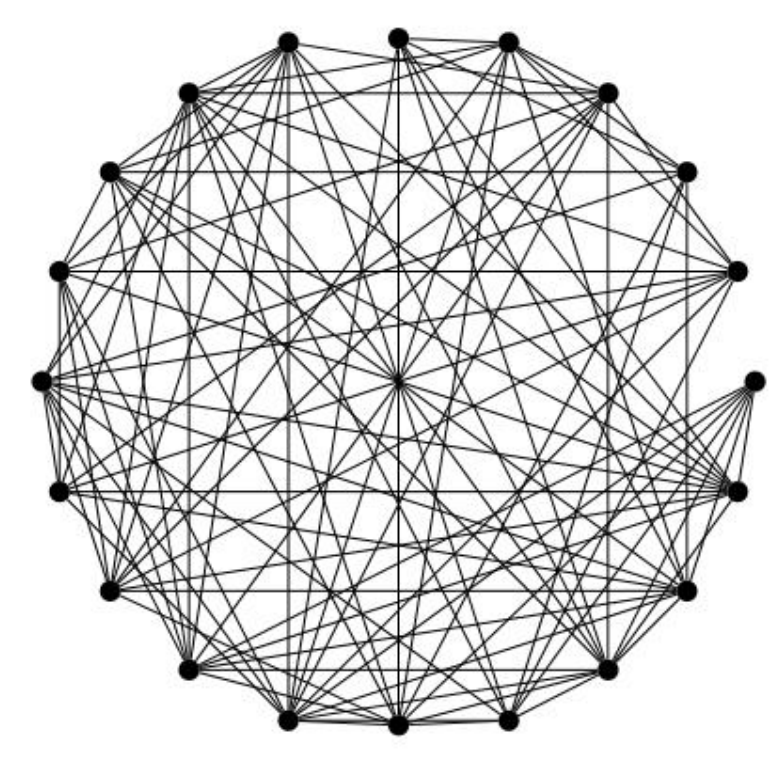

FIGURE 1.4. A random graph $G\left(20, \frac{1}{2}\right)$. 
As we often use asymptotic notation to present results, we will introduce some main asymptotic notation. Let $f(x)$ and $g(x)$ be two functions defined on some subset of the real numbers, we can write: $f(x)=O(g(x))$ if

$$
\lim _{x \rightarrow \infty} \sup \frac{f(x)}{|g(x)|}
$$

exists and is finite. It is equivalent that there exists a constant $c$ and a real number $x_{0}$, such that for all $x>x_{0}$,

$$
f(x) \leq c g(x)
$$

Similarly, we write: $f(x)=o(g(x))$, if

$$
\lim _{x \rightarrow \infty} \frac{f(x)}{g(x)}=0 .
$$

Another asymptotic notation we introduce is

$$
f(x)=\theta(g(x))
$$

that is, there exists positive constants $c_{1}$ and $c_{2}$, and a real number $x_{0}$, such that for all $x>x_{0}$,

$$
c_{1}|g(x)| \leq|f(x)| \leq c_{2}|g(x)|
$$


1.2. Power law degree distribution. Power law degree distributions are one of the most prominent properties of OSNs and other complex networks (see [3]). Analysis and experiments on OSNs found that the degree distribution of vertices follows a power law, which means that a large proportion of vertices have low degree, but a small (but non-negligible) proportion of vertices have substantially higher degree. In particular, the number of vertices of degree $k$ (where $k$ is non-negative integer) is proportional to an inverse power of $k$. We define the number of vertices of degree $k$ as:

$$
N_{k}=\left|\left\{x \in V(G): \operatorname{deg}_{G}(x)=k\right\}\right| .
$$

Then we have the formula:

$$
\frac{N_{k}}{N} \sim k^{-\beta},
$$

where $\beta>2$ is a positive real number, $N$ is the order of graph $G$. If we take the logarithms on (1), then we can express the power law as:

$$
\log N_{k} \sim \log N-\beta \log (k),
$$

which gives a line with the slope of negative $\beta$.

In 2006, Kumar et al. found that the degree distribution of the Flickr OSN follows a power law (see [12]). Note that the power law 
degree distribution may only fit a certain range of degrees; for small and large degree vertices, the log-log plot may show more noise. We analyzed Wikipedia sample data and checked the degree distribution. Figure 1.5 shows that the degree distribution log-log plot of Wikipedia follows a power law, we can see that log-log plot of out-degree of those low degree vertices follows a straight line.

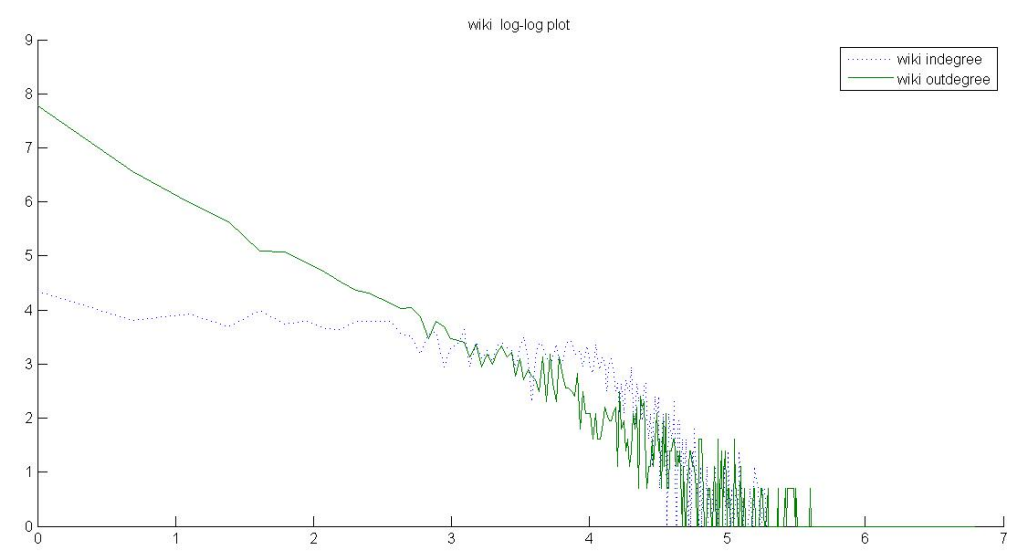

FiguRE 1.5. Wikipedia degree distribution log-log plot.

1.3. Small world property. The small world property is an important feature of OSNs, which was implicitly introduced by Milgram (see [15]), and explicitly by Watts and Strogatz (see [21]). The neighbours of a vertex $x$ are defined as all vertices which are joined to $x$. We use notation $N(x)$ to represent the neighbour set of vertex $x$. We define the distance between pairs of vertices as the number of edges of 
a shortest path joining them. We use notation $d(x, y)$ to present the distance between vertices $x$ and $y$. In the example above of Figure 1.2 . we have that

$$
\begin{gathered}
N(x)=\{y, u, v\}, N(y)=\{x, u\} \\
d(x, y)=d(x, u)=d(x, v)=d(y, u)=d(u, v)=1, \\
d(y, v)=2 .
\end{gathered}
$$

We now give the definition of diameter, average distance, and clustering coefficient. The diameter of a graph is defined as the maximum distance between any two vertices (and $\infty$ if the graph is disconnected). We usually use the notation $\operatorname{diam}(G)$ to represent the diameter of graph $G$. We define the average distance of an undirected graph $G$ of order $N$ as

$$
D(G)=\frac{\frac{1}{2} \sum_{x, y \in V(G)} d(x, y)}{\left(\begin{array}{c}
N \\
2
\end{array}\right)}
$$

Let $S$ be the subgraph induced by $N(x)$ and $x$, and let $|E(S)|$ be the number of edges in S. For a given vertex $x$ in $G$ with degree at least two define the clustering coefficient of $x$ as

$$
C(x)=\frac{|E(S)|}{\left(\begin{array}{c}
\operatorname{deg}_{S}(x) \\
2
\end{array}\right)}
$$


The clustering coefficient of $G$ is the average of the clustering coefficients over all vertices of $G$, written $C(G)$, where

$$
C(G)=\frac{1}{|V(G)|} \sum_{x \in V(G)} C(x)
$$

With the graph of Figure 1.2 diam $(G)=2$, and $D(G)=\frac{7}{12}$. Further, $C\left(v_{1}\right)=C\left(v_{3}\right)=\frac{5}{3}, C\left(v_{2}\right)=C\left(v_{4}\right)=2$, and $C(G)=\frac{11}{6}$.

The small world property demands a low average distance of $O(\log \log N)$ (or a diameter of $O(\log N))$, and a higher clustering coefficient than found in a random graph $G(N, p)$ with the same order $N$ and approximately same average degree.

1.4. Bad spectral expansion. Social networks often organize into separate clusters in which the intra-cluster links are significantly higher than the number of inter-cluster links. It is reported that social networks possess bad spectral expansion properties realized by small gaps between the first and second eigenvalues of their adjacency matrices (see [10]).

An adjacency matrix is a numerical way to present a graph. For a graph of order $N$, it is an $N \times N$ square matrix with binary entries. That is, entry $a_{i j}$ can take the value of either 0 or 1 , with 1 if there is an edge between the $i$ th and $j$ th vertices, and 0 if there is no edge 
between the $i$ th and $j$ th vertices. With the graph of Figure 1.2 , and the order of $\left\{v_{1}, v_{2}, v_{3}, v_{4}\right\}$, we can write adjacency matrix as:

$$
A=\left(\begin{array}{llll}
0 & 1 & 1 & 1 \\
1 & 0 & 1 & 0 \\
1 & 1 & 0 & 1 \\
1 & 0 & 1 & 0
\end{array}\right) .
$$

A nonzero scalar $\lambda$ and nonzero vector $\mathbf{v}$, which satisfy

$$
A \mathbf{v}=\lambda \mathbf{v}
$$

are called the eigenvalues and eigenvectors, respectively, of the matrix $A$. Note that if $G$ is undirected, then its adjacency matrix is symmetric and so has all real eigenvalues. We sort the eigenvalues of matrix $A$ such that

$$
\left|\lambda_{1}\right| \geq\left|\lambda_{2}\right| \geq \ldots \geq\left|\lambda_{N-1}\right| \geq\left|\lambda_{N}\right|
$$

Here $\lambda_{1}$ and $\lambda_{2}$ are called the first and second eigenvalues of $G$. With the undirected graph of Figure 1.2 , we have that $\lambda_{1}=2.56$ and $\lambda_{2}=0$.

Fix a graph $G$ of order $N$ with first and second eigenvalues $\lambda_{1}$ and $\lambda_{2}$, respectively. Suppose that $p$ is chosen so that the random graph $G(N, p)$ has the same expected average degree as $G$, and let $\rho_{1}$ and $\rho_{2}$ be the first and second eigenvalues of $G(N, p)$, respectively. We say 
that $G$ has bad spectral expansion if

$$
\left|\lambda_{1}-\lambda_{2}\right|<\left|\rho_{1}-\rho_{2}\right|
$$

\section{Overview of Thesis}

In this thesis, the remaining chapters focus on the following topics. In Chapter 2, we introduce the Geometric Protean models for OSNs, written GEO-P (first presented in [7]). We summarize the main theoretical results proved about this model in [7]. We simulate graphs using GEO-P, analyze their properties, and verify if graphs generated by those models follow the observed OSNs properties, such as power law degree distribution, small world property, and bad spectral expansion. In Chapter 3, we present results on real OSNs data, compute properties of graphs simulated by sampled data, and verify if the OSN samples obey the main properties described in Chapter 1. In Chapter 4, we introduce and simulate new models based on the GEO-P model. We compare these models to real OSN data. In Chapter 5, we summarize the results of the previous chapters, and state open problems.

We note that the results of this thesis are original work. Parts of Chapters 2 were included in the accepted paper [7]. 


\section{CHAPTER 2}

\section{Geometric models for OSNs}

In this chapter, we introduce the Geometric Protean (or GEO-P) model for OSNs, first presented in [7].

\section{Introduction to the GEO-P model}

The GEO-P model has vertices in a fixed metric space. We choose $N$ vertices in a $m$-dimensional Euclidean space by some random process, assign vertices integers as their ranks, and determine edges by the probability based on ranks of vertices.

There are four parameters in this model: the attachment strength $\alpha \in(0,1)$, the density parameter $\beta \in(0,1-\alpha)$, the dimension $m \in \mathbb{N}$, and the link probability $p \in(0,1]$. The GEO-P model generates a sequence of graphs $G_{t}$ over an infinite sequence of discrete time-steps. At each time-step $t$, one new vertex is born and one existing vertex dies. For each vertex $x$, we define a radius of influence, at each timestep we place an edge between pairs of vertices with probability $p$ if

one lies within the other's radius of influence. We begin by defining 
$r(x, t)$ to indicate the (unique) rank of vertex $x$ at time-step $t$. Hence,

$$
r(x, t) \in[N],
$$

where $[N]=\{1,2,3, \ldots, N-1, N\}$. In this model, 1 is the highest rank and $N$ is the lowest rank. At initial time-step $t=0$, vertices are assigned ranks by a random order. Figure 2.1 shows 20 randomly chosen vertices in the 2-dimensional unit hypercube with ranks assigned by a random order.

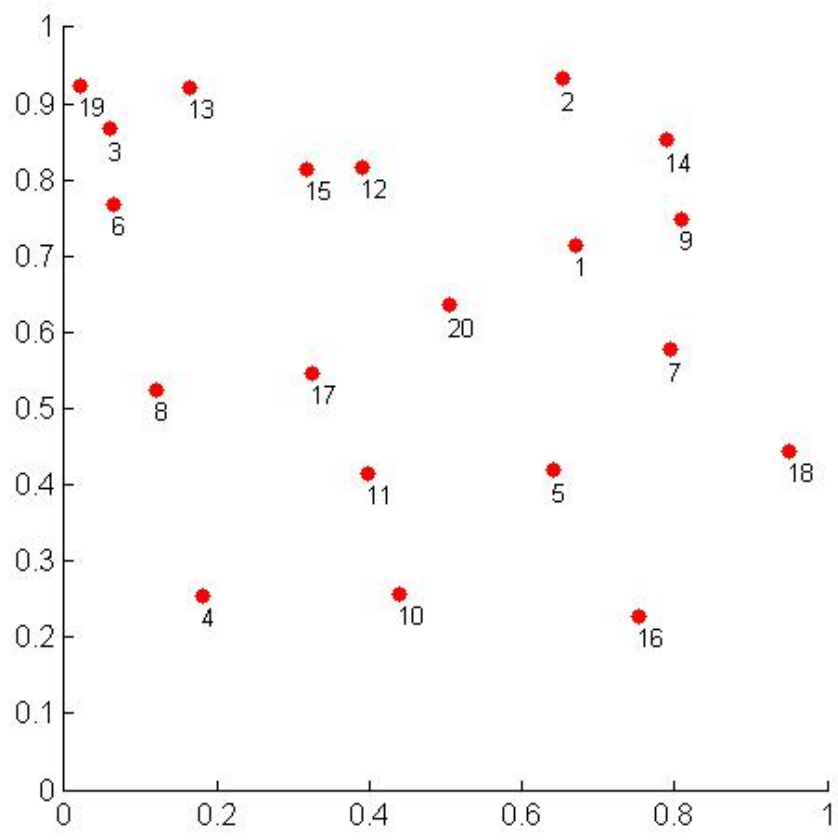

FiguRE 2.1. 20 randomly chosen vertices in a 2dimensional unit hypercube. 
At time-step $t$, where $t>0$ the new vertex $u$ is assigned a rank randomly chosen from set $[N]$, written $r(u, t)$. We name the deleted vertex as $v$, and its rank at time-step $t-1$ is written as $r(v, t-1)$. We assign ranks to vertices $x$ at time-step t by

$$
r(x, t)=r(x, t-1)+\delta-\gamma,
$$

where $x \in V\left(G_{t-1}\right) \cap V\left(G_{t}\right), \delta=1$ if $r(x, t-1)>r(u, t)$, and 0 , otherwise, and $\gamma=1$ if $r(x, t-1)>r(v, t-1)$, and 0 , otherwise. Every vertex has a unique rank at a given time-step; that is, $r(x, t)=r(y, t)$ if and only if $x=y$.

We define the influence region of vertex $x$ at time-step $t \geq 0$, written $R(x, t)$, to be the ball around $x$ with volume

$$
|R(x, t)|=r(x, t)^{-\alpha} N^{-\beta}
$$

Since the exponent $-\alpha$ is negative, we have that the vertex with rank 1 has the largest influence region, and vertex with rank $N$ has the smallest influence region. We assume that the volume of influence region corresponds to the ability to gain edges. Hence, higher ranked vertices are more likely to receive more edges than lower rank vertices. In Table 1 we list ranks, coordinates, and influence region volume of 
20 vertices which are randomly chosen in Figure 2.1, where we choose $\alpha=0.7$ and $\beta=0.15$.

TABLE 1. Ranks, coordinates, and influence region volume of 20 vertices in the 2-dimensional unit hypercube.

\begin{tabular}{|c|c|c|}
\hline rank & coordinates & volume \\
\hline 1 & $(0.6712,0.7152)$ & 0.137 \\
\hline 2 & $(0.6537,0.9326)$ & 0.1488 \\
\hline 3 & $(0.06,0.8668)$ & 0.0878 \\
\hline 4 & $(0.1807,0.2554)$ & 0.1191 \\
\hline 5 & $(0.6421,0.419)$ & 0.2418 \\
\hline 6 & $(0.0642,0.7673)$ & 0.0812 \\
\hline 7 & $(0.7947,0.5774)$ & 0.3928 \\
\hline 8 & $(0.1202,0.525)$ & 0.1059 \\
\hline 9 & $(0.8092,0.7486)$ & 0.1634 \\
\hline 10 & $(0.44,0.2576)$ & 0.1273 \\
\hline 11 & $(0.3989,0.4151)$ & 0.0916 \\
\hline 12 & $(0.3908,0.8161)$ & 0.182 \\
\hline 13 & $(0.1635,0.9211)$ & 0.638 \\
\hline 14 & $(0.7891,0.8523)$ & 0.2068 \\
\hline 15 & $(0.3174,0.8145)$ & 0.1121 \\
\hline 16 & $(0.7519,0.2287)$ & 0.0958 \\
\hline 17 & $(0.3258,0.5464)$ & 0.1006 \\
\hline 18 & $(0.9509,0.444)$ & 0.0784 \\
\hline 19 & $(0.0205,0.9237)$ & 0.0844 \\
\hline 20 & $(0.5056,0.6357)$ & 0.2957 \\
\hline
\end{tabular}

We use $\|\cdot\|_{\infty}$ to indicate the infinity norm or maximum norm, defined as:

$$
\|\mathbf{v}\|_{\infty}=\max \left(\left|v_{1}\right|,\left|v_{2}\right|, \ldots,\left|v_{m-1}\right|,\left|v_{m}\right|\right)
$$

where $\mathbf{v}$ is a vector in $\mathbb{R}^{m}$, and $\mathbf{v}=\left(v_{1}, v_{2}, \ldots, v_{m-1}, v_{m}\right) \in \mathbb{R}^{m}$. Let $S$ be the unit hypercube in $\mathbb{R}^{m}$. We define the torus metric on $S$ as 
follows. For $x, y \in S$ define

$$
d(x, y)=\min \left\{\|x-y+u\|_{\infty}: u \in\{-1,0,1\}^{m}\right\} .
$$

The torus metric thus "wraps around" the boundaries of the unit cube, so every point in $S$ is equivalent. The torus metric is chosen so that there are no boundary effects, and altering the metric will not significantly affect the main results. With the example of Figure 2.1,

$$
d(17,11)=0.1314, d(17,5)=0.3162 .
$$

We define the $m$-dimensional radius of a vertex in terms of a parameter $C_{m}$ as

$$
\operatorname{Rad}(x)=\left(\frac{R(x, t)}{C_{m}}\right)^{\frac{1}{m}}
$$

where, if $m$ is even, then

$$
C_{m}=\frac{\pi^{\frac{m}{2}}}{\left(\frac{m}{2}\right) !}
$$

and if $m$ is odd, then

$$
C_{m}=\frac{2^{\frac{m+1}{2}} \pi^{\frac{m-1}{2}}}{m ! !} .
$$


Here, $m$ !! is defined as double factorial of an odd integer $m$; that is, for $m=2 k-1$, and $k$ a positive integer:

$$
m ! !=\prod_{i=1}^{k}(2 i-1)=\frac{(2 k) !}{k ! 2^{k}}
$$

We apply (5) to calculate the influence region radius for vertices 17, 11 and 5; Table 2 shows their radius values. If we simply choose parameter $p=1$, then for every pair of vertices there will be an edge if at least one of those two verties falls in another one's influence region. Vertex 11 falls in 17's influence region as depicted in Figure 2.2|(a), so there is an edge between them; vertices 17 and 5 are too far apart to fall in each other's influence region as Figure 2.2 (b) showed. Hence, no edge is between vertices 17 and 5; see Figure 2.3 .
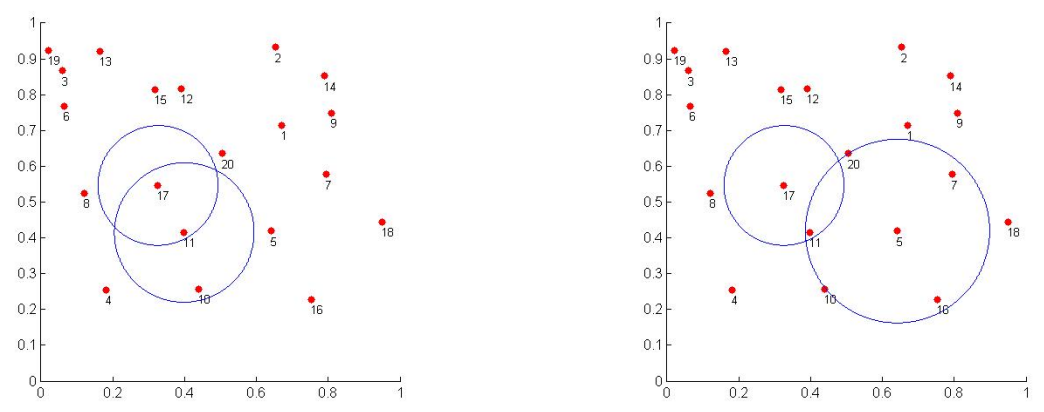

(a) Influence region of vertices 17 and 11. (b) Influence regions of vertices 17 and 5 .

FiguRE 2.2. Graph generated by 20 vertices at $p=1$. 


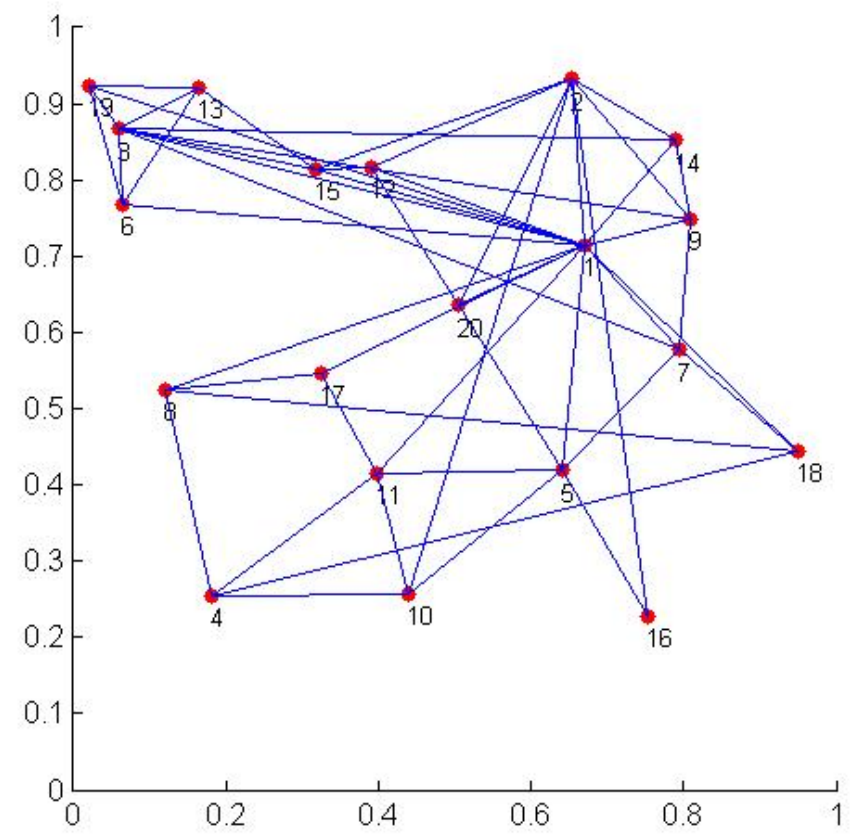

Figure 2.3. Graph generated by the GEO-P model, $\alpha=0.7, \beta=0.15$, and $p=1$.

TABLE 2. Radius of vertices 17,11 , and 15 .

\begin{tabular}{|c|c|c|c|}
\hline rank & coordinates & volume & radius \\
\hline 17 & $(0.325834,0.546449)$ & 0.087807 & 0.1672 \\
\hline 11 & $(0.398881,0.415093)$ & 0.119089 & 0.1947 \\
\hline 5 & $(0.642061,0.419048)$ & 0.2068 & 0.2566 \\
\hline
\end{tabular}

We now describe precisely how the GEO-P model generates graphs.

1. At the initial time-step $t=0$, we randomly choose $N$ vertices in $S$, and randomly assign vertex unique rank chosen from set $[N]$. An edge between pair of vertices $x$ and $y$ is created with probability $p$, 


$$
d(x, y) \leq \max \{\operatorname{Rad}(x), \operatorname{Rad}(y)\}
$$

2. At time-step $t>0$, a randomly chosen vertex $v \in V\left(G_{t-1}\right)$ dies, a new vertex $u$ is born and is assign a rank randomly chosen from set $[N]$. We update ranks of vertices $x \in V\left(G_{t-1}\right) \cap V\left(G_{t}\right)$ by (2), and determine edges by influence region, distance and probability $p$.

It can be shown that this stochastic process converges to a stationary distribution (see [7]). The random graph corresponding to this distribution with given parameters $\alpha, \beta, m, p$ is called the geo-protean (or GEO-P model) graph, and is written $\operatorname{GEO}-\mathrm{P}(\alpha, \beta, m, p)$.

\section{Results on GEO-P}

We now introduce some properties of graphs generated by the GEO-P model. Recall that an event holds asymptotically almost surely (a.a.s.) if it holds with probability tending to 1 as $n$ tends to infinity. The following theoretical results for the GEO-P model were stated and proved in [7].

Let $N_{k}=N_{k}(m, p, \alpha, \beta)$ denote the number of vertices of degree $k$ in a graph of order $n$, generated by the GEO-P model with parameters $p, \alpha$, and $\beta$. Define $N_{\geq k}=\sum_{l \geq k} N_{l}$ to be the number of vertices of 
degree greater than or equal to $k$. The following theorem shows that the GEO-P model generates graphs with power law degree distribution.

Theorem $2.1([7])$. Let $\alpha \in(0,1), \beta \in(0,1-\alpha), m \in \mathbb{N}, p \in(0,1]$, and

$$
n^{1-\alpha-\beta} \log ^{1 / 2} n \leq k \leq n^{1-\alpha / 2-\beta} \log ^{-2 \alpha-1} n .
$$

Then a.a.s. $G E O-P(\alpha, \beta, m, p)$ satisfies

$$
N_{\geq k}=\left(1+O\left(\log ^{-1 / 3} n\right)\right)\left(\frac{\alpha}{\alpha+1}\right) p^{1 / \alpha} n^{(1-\beta) / \alpha} k^{-1 / \alpha}
$$

From (6), for fixed parameters $\alpha$ and $\beta$, we have that

$$
\frac{N_{\geq k}}{n} \sim k^{-\frac{1}{\alpha}}
$$

Hence,

$$
\frac{N_{k}}{n} \sim k^{-\frac{1}{\alpha}-1}
$$

(see, for example, [3]).

Theorem 2.1 demonstrates that for a range of degrees, the GEO-P model generates power law graphs with exponent

$$
b=1+1 / \alpha .
$$


For a graph $G=(V(G), E(G))$ of order $n$, define the average degree of $G$ by $d=\frac{2|E(G)|}{n}$. The second theoretical results shows that graphs generated by the GEO-P model are dense; that is, the average degree tends to infinity with $n$.

Theorem 2.2 ([7]). A.a.s. the average degree of $\operatorname{GEO-P}(\alpha, \beta, m, p)$ is

$$
d=(1+o(1))\left(\frac{p}{1-\alpha}\right) n^{1-\alpha-\beta} .
$$

By the small world property, OSNs require a diameter of $O(\log n)$. Following theorem describes the diameter features of graphs generated by GEO-P model.

Theorem $2.3([7])$. Let $\alpha \in(0,1), \beta \in(0,1-\alpha), m \in \mathbb{N}$, and $p \in(0,1]$. Then a.a.s. the diameter of $\operatorname{GEO} P(\alpha, \beta, m, p)$ is

$$
D=O\left(n^{\frac{\beta}{(1-\alpha) m}} \log ^{\frac{2 \alpha}{(1-\alpha) m}} n\right) .
$$

If we assume that the dimension $m$ grows as a logarthmic function of $n$, then the diameter of the GEO-P model is constant. To see this, 
if we let $m=C \log n, C \in(0,1)$, then we have by $(9)$ that

$$
\begin{aligned}
\log D & =O\left(\frac{\beta}{(1-\alpha) C \log n} \log n+\frac{2 \alpha}{(1-\alpha) C \log n} \log \log n\right) \\
& =O\left(\frac{\beta}{(1-\alpha) C \log n} \log n+o(1)\right) \\
& =O\left(\frac{\beta}{(1-\alpha) C}\right) .
\end{aligned}
$$

If $\xi=\frac{\beta}{(1-\alpha) C}$, then we have that

$$
\begin{aligned}
\log D & =O(\xi) \\
& =O(1) .
\end{aligned}
$$

Hence,

$$
D=\exp (O(1))=O(1)
$$

Recall that another main feature of small world property is a higher clustering coefficient than found in random graphs with the same expected average degree. The next theorem proves that this does indeed hold in the GEO-P model. 
Theorem $2.4([7])$. A.a.s. the clustering coefficient of $G$ sampled from GEO-P $(\alpha, \beta, m, p)$ satisfies the following inequality:

$$
\begin{aligned}
C(G) & \geq(1+o(1))\left(\frac{3}{4}\left(1-\frac{2}{3 K}\right)\right)^{m}\left(\frac{1-\alpha}{1+\alpha}\right) p \\
& =(1+o(1)) \exp \left(O\left(\frac{m}{K}\right)\right)\left(\frac{3}{4}\right)^{m}\left(\frac{1-\alpha}{1+\alpha}\right) p
\end{aligned}
$$

where

$$
K=\left\lfloor\left(\frac{n^{1-\alpha-\beta}}{\log ^{3} n}\right)^{1 / m}\right\rfloor-\delta,
$$

with $\delta$ either 0 or 1 so $K$ is even.

For example, when $m=o(\log n)$ it can be shown that a.a.s.

$$
C(G) \geq\left(\frac{3}{4}\right)^{m+o(m)}=n^{o(1)} .
$$

Note that for random graph $G(n, p)$, the clustering coefficient of a vertex satisfies $C(x)=p$ (see [3] $)$. Hence,

$$
C(G(n, p))=\frac{1}{n} \sum_{x \in V(G)} C(x)=p .
$$

Hence,

$$
C(G) \geq n^{o(1)} \gg C(G(n, d / n))=d / n .
$$


That is, the clustering coefficient of graphs generated by the GEO-P model is larger than in a random graph with the same order.

Let $A$ denote the adjacency matrix and $D$ denote the diagonal degree matrix of a graph $G$. Then the normalized Laplacian of $G$ is

$$
\mathcal{L}=I-D^{-1 / 2} A D^{-1 / 2} .
$$

Let $0=\lambda_{0} \leq \lambda_{1} \leq \cdots \leq \lambda_{n-1} \leq 2$ denote the eigenvalues of $\mathcal{L}$. The spectral gap of the normalized Laplacian is defined as

$$
\lambda=\max \left\{\left|\lambda_{1}-1\right|,\left|\lambda_{n-1}-1\right|\right\} .
$$

We state that random graphs have good spectral expansion, since $\lambda=$ $o(1)$ (see $[\mathbf{8}, \mathbf{9}])$. The next theorem shows that graphs generated by the GEO-P model have bad expansion properties, compared to random graphs.

Theorem $2.5([7])$. Let $\alpha \in(0,1), \beta \in(0,1-\alpha), m \in \mathbb{N}$, and $p \in(0,1]$. Let $\lambda(n)$ be the spectral gap of the normalized Laplacian of GEO-P $(\alpha, \beta, m, p)$. Then a.a.s we have the following.

(1) If $m=m(n)=o(\log n)$, then $\lambda(n)=1+o(1)$. 
(2) If $m=m(n)=C \log n$ for some $C>0$, then

$$
\lambda(n) \geq 1-\exp \left(-\frac{\alpha+\beta}{C}\right)
$$

Note that in either case (1) or $(2), \lambda(n)$ is close to 1 , unlike in the random graph $G(n, p)$ (where $\lambda(n)$ tends to 0 ).

\section{Dimension of the GEO-P model}

Given an OSN, its dimension is the minimum number $m$ of attributes needed to identify users or a small set of users. Here attributes correspond to location, career, or hobbies, for example. We think that users with similar attributes are more likely to be friends. Hence, there should be an embedding of the OSN in $m$-dimensional Euclidean space where users with similar attributes are clustered together.

The results stated for the GEO-P model in the previous section point to a theoretical estimate of the dimension of an OSN. The Logarithmic Dimension Hypothesis (or $L D H$ ) states that the dimension $m$ of an OSN satisfies

$$
m=O(\log n)
$$

Let the OSN order to be $n$, power law exponent to be $b$, average degree to be $d$, and let $D$ be the diameter. Then (7) shows us how to estimate 
for $\alpha$ based on the power law exponent $b$. Let

$$
\begin{aligned}
d^{*} & =\log d / \log n, \\
D^{*} & =\log D / \log n .
\end{aligned}
$$

Equations (8) and (9) imply that (ignoring constants)

$$
\begin{aligned}
d^{*} & =1-\alpha-\beta, \\
D^{*} & =\frac{\beta}{(1-\alpha) m} .
\end{aligned}
$$

Thus, by (10) and (11) we estimate $m$ as:

$$
\begin{aligned}
m & =\frac{1}{D^{*}}\left(1-\left(\frac{b-1}{b-2}\right) d^{*}\right) . \\
& =\frac{\log n}{\log D}(1-o(1))
\end{aligned}
$$

If $D$ is a constant, then (12) suggests that the dimension $m$ depends on $\log n / \log D$. That is, $m$ grows logarithmically with $n$. Hence, support for the LDH comes from the GEO-P model, although it is yet to be proved in practice (see Section 3 of Chapter 3).

Table 3 predicts the dimensions of OSNs Cyworld, Flickr, Twitter, and YouTube based on (12) (see [7]). Let $b$ be the power law exponent 
for the in-degree distribution, $n$ be the order of given OSN, and let $m$ be rounded up to the nearest integer.

TABle 3. Dimensions of the OSNs: Cyworld, Flickr, Twitter, and YouTube.

\begin{tabular}{|l||l|l|l|l|}
\hline Parameter & OSN & & & \\
\hline & Cyworld & Flickr & Twitter & YouTube \\
\hline \hline$n$ & $2.4 \times 10^{7}$ & $3.2 \times 10^{7}$ & $7.5 \times 10^{7}$ & $3 \times 10^{8}$ \\
\hline$b$ & 5 & 2.78 & 2.4 & 2.99 \\
\hline$d^{*}$ & 0.22 & 0.17 & 0.17 & 0.1 \\
\hline$D^{*}$ & 0.11 & 0.19 & 0.1 & 0.16 \\
\hline$m$ & 7 & 4 & 5 & 6 \\
\hline
\end{tabular}




\section{CHAPTER 3}

\section{Properties of Real OSN Data}

In this chapter, we consider data sampled from real OSNs. We analyze their properties viewed as complex networks, and contrast them to graphs simulated by the GEO-P model. Our sampled data was retrieved from the OSNs Wikipedia (see [18]) and YouTube (see [16]). Their orders are 7,115 and 570,774, respectively, and there are 103,689 and 4,945,382 edges in those two samples samples, respectively. We mainly focus on investigating their degree distribution, spectral gap and diameter.

\section{Properties of Real OSNs}

In Chapter 1, we have shown that the Wikipedia sample follows a power law degree distribution. Figure 3.1 shows the degree distribution of a graph sampled from YouTube with order 570,774 (see [16]). It is evident that it follows a power law degree distribution. Figure 3.2 shows that the log-log plot is a straight line for medium degree vertices; that is, informally, vertices which are not of low or high degree. 


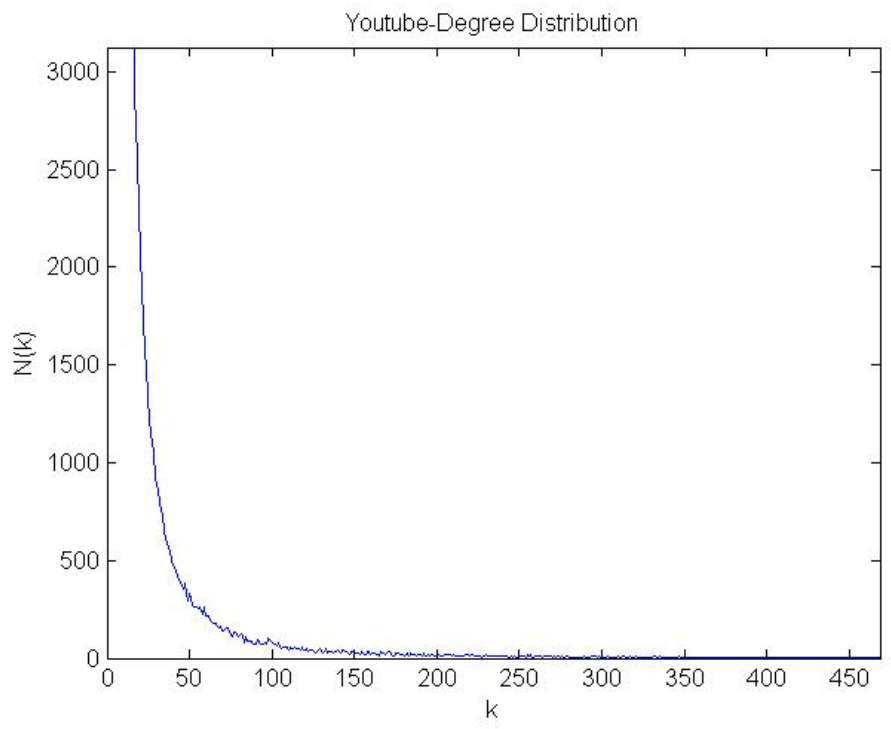

Figure 3.1. YouTube sample degree distribution.

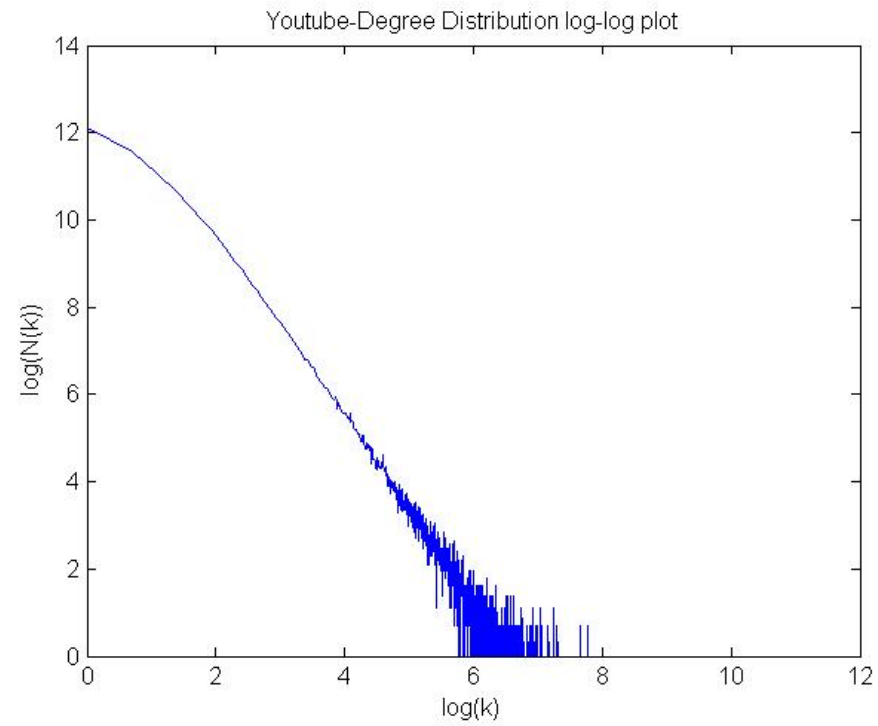

Figure 3.2. YouTube sample degree distribution log$\log$ plot.

We next analyzed OSN data for their diameters. We considered graph samples from Wikipedia and YouTube. We applied the software 
SNAP (see [18]) to calculate the diameters, and calculated that they are 10 and 23 , respectively.

Finally, we checked the spectral gap of graphs in our OSNs samples. To evaluate the spectral gap, we calculate the first and second eigenvalues of their adjacency matrix. OSNs have large-scale adjacency matrices. To make the calculations more efficient, we applied the power method (see [3]) to approximate the first eigenvalue $\lambda_{1}$. Let $A$ be the $N \times N$ adjacency matrix, and let $\mathbf{v}_{\mathbf{1}}^{(\mathbf{0})}$ be any $N \times 1$ vector (here we let $\left.\mathbf{v}_{\mathbf{1}}^{(\mathbf{0})}=(1,1, \ldots, 1)\right)$. Then we have the iteration:

$$
\begin{aligned}
\mathbf{v}_{\mathbf{1}}^{(\mathbf{k}+\mathbf{1})} & =\frac{A \mathbf{v}_{\mathbf{1}}^{(\mathbf{k})}}{\left\|A \mathbf{v}_{\mathbf{1}}^{(\mathbf{k})}\right\|}, \\
\lambda_{1}^{(k+1)} & =\frac{A \mathbf{v}_{\mathbf{1}}^{(\mathbf{k}+\mathbf{1})}}{\mathbf{v}_{\mathbf{1}}^{(\mathbf{k}+\mathbf{1})}} .
\end{aligned}
$$

We run this iteration until $\left|\frac{\lambda_{1}^{k+1}-\lambda_{1}^{k}}{\lambda_{1}^{k+1}}\right|<\epsilon$, where $\epsilon$, as the accuracy control, is a fixed positive real number.

To approximate the second eigenvalue, we apply the deflation method (see [4]). Let $\lambda_{1}$ and $\mathbf{v}_{\mathbf{1}}$ be the largest eigenvalue and eigenvector, respectively, and let $A$ still be the adjacency matrix, then we have that

$$
A^{*}=A-\lambda_{1} \mathbf{v}_{\mathbf{1}} \mathbf{v}_{\mathbf{1}}^{\mathbf{T}},
$$

where $\mathbf{v}_{\mathbf{1}}^{\mathbf{T}}$ is the transpose of $\mathbf{v}_{\mathbf{1}}$. 
Next, we apply power method to $A^{*}$ to compute its largest eigenvalue. This in turn should be the second largest eigenvalue of the initial matrix $A$.

Using these eigenvalue approximation algorithms, we obtain that the spectral gap of the Wikipedia sample is about

$$
\left|\lambda_{1}-\lambda_{2}\right|=45.1290-26.9872=18.1419 .
$$

We generated a random graph with the same order to contrast this result. The spectral gap of random graph of the same order $G(7115,1 / 2)$ is approximately

$$
\left|\rho_{1}-\rho_{2}\right|=3499.9-82.8528=3417.0472 .
$$

We now introduce the first and second neighbour set. The first neighbour set of $x$, written $N(x)=N_{1}(x)$, consists of the neighbours of $x$. Correspondingly, the second neighbour set of $x$, written $N_{2}(x)$ is the set of vertices with distance two from $x$.

We consider ten subgraphs $S$ of YouTube by sampling first and second neighbour sets. More precisely, we first choose a vertex $x$ uniformly at random from the YouTube graph. We consider the subgraph be induced by the closed first and second neighbour sets of $x$; that is, 
the subgraph induced by

$$
\{x\} \cup N_{1}(x) \cup N_{2}(x) .
$$

Table 1 shows the spectral gaps of our YouTube samples, and the spectral gaps of random graphs with the same order (to contrast with our OSN samples).

TABLE 1. Spectral gaps of YouTube samples and random graphs of same order.

\begin{tabular}{|c|c|c|}
\hline order & \multicolumn{2}{|c|}{ gap } \\
\hline & YouTube & random graph \\
\hline \hline 4497 & 13.28 & 2193.76 \\
\hline 5505 & 53 & 2696.47 \\
\hline 5503 & 12.63 & 2680.92 \\
\hline 6409 & 22.38 & 3115.31 \\
\hline 5897 & 10.9 & 2923.18 \\
\hline 3528 & 18.39 & 1727.80 \\
\hline 7507 & 20.56 & 3619.51 \\
\hline 6089 & 14.57 & 2997.98 \\
\hline 5053 & 15.56 & 1709.70 \\
\hline 6788 & 60.8 & 3223.98 \\
\hline
\end{tabular}

With OSNs Wikipedia and YouTube as samples, the above calculation results suggest that OSNs have the following properties.

1. For some range of degree vertices (mostly, medium degree vertices), OSNs follow a power law degree distribution.

2. OSNs have a small diameter, which is one of the main features of the small world property. 
3. OSNs have smaller spectral gaps than a random graph, which indicates OSNs have bad spectral expansion.

\section{Properties of Graphs Generated by the GEO-P Model}

We now simulate graphs using the GEO-P model in several dimensions, and compare and contrast these samples with real OSN data. Once again, we measure the degree distribution, diameter, and spectral gap.

Figures 3.3 and 3.4 show the degree distributions of graphs generated by the GEO-P model, where the order $N$ is 7115 (the same order as our Wikipedia samples). We used dimensions $m$ from 1 to 5 , and we used two sets of values for parameters $\alpha$ and $\beta$. We can see that for the medium degree vertices, the log-log plot is a straight line, which suggests that graphs generated by the GEO-P model follow a power law degree distribution.

Table 2 displays the spectral gap of graphs generated by the GEO-P model. Recall that we calculated a spectral gap of a sampled random graph $G(7115,1 / 2)$ to be 3417.0472 (see Section 3.1). We may conclude that graphs generated by the GEO-P model have small spectral gap. Correspondingly, they have bad spectral expansion. 


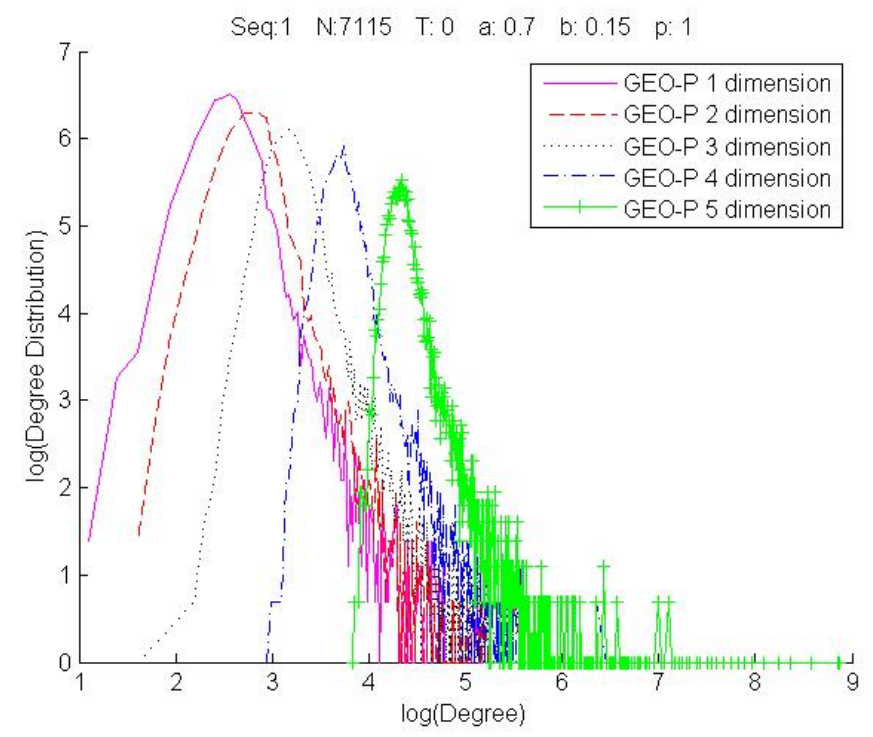

FiguRe 3.3. GEO-P degree distribution log-log plot, $N=7115, \alpha=0.7, \beta=0.15, p=1$.

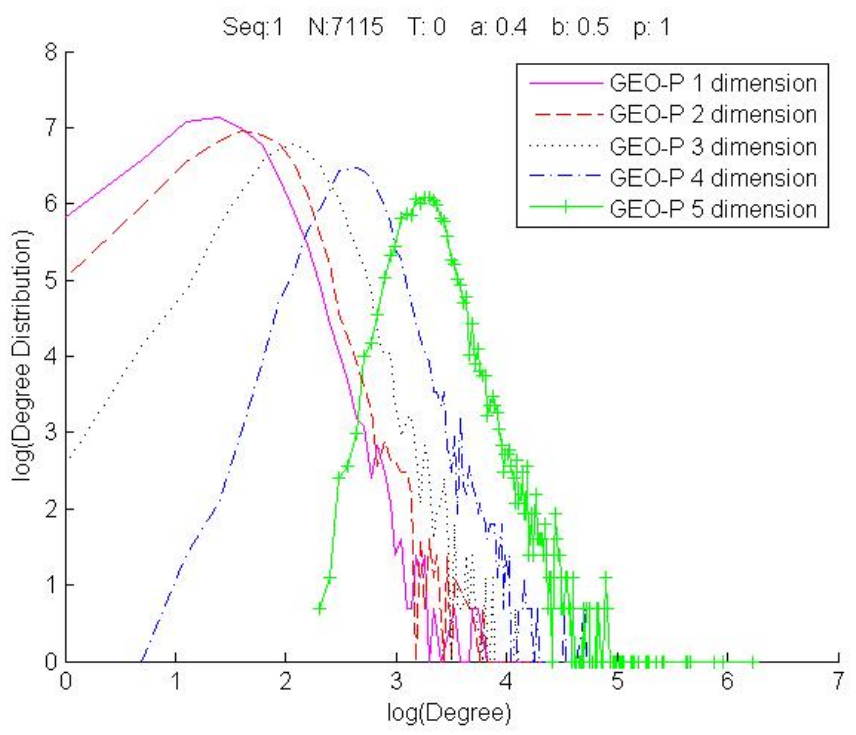

Figure 3.4. GEO-P degree distribution log-log plot, $N=7115, \alpha=0.4, \beta=0.5, p=1$.

Table 3 displays the diameters of graphs generated by the GEO-P model. We can see that graphs generated by the GEO-P model have small diameter, and the diameter decreases as $m$ increases. 
TABLE 2. Spectral gaps of graphs generated by the GEO-P model.

\begin{tabular}{|c|c||c|c|}
\hline \multicolumn{4}{|c|}{$N=7115, p=1$} \\
\hline \multicolumn{2}{|c|}{$\alpha=0.7, \beta=0.15$} & \multicolumn{2}{|c|}{$\alpha=0.4, \beta=0.5$} \\
\hline dimension & gap & dimension & gap \\
\hline \hline 1 & 23.700163 & 1 & 0.070531 \\
\hline 2 & 35.04388 & 2 & 0.028725 \\
\hline 3 & 57.751959 & 3 & 0.380326 \\
\hline 4 & 48.840852 & 4 & 3.21985 \\
\hline 5 & 95.301035 & 5 & 4.555701 \\
\hline
\end{tabular}

TABLE 3. Diameters of graphs generated by the GEO-P model.

\begin{tabular}{|c|c||c|c|}
\hline \multicolumn{4}{|c|}{$N=7115, p=1$} \\
\hline \multicolumn{2}{|c|}{$\alpha=0.7, \beta=0.15$} & $\alpha=0.4, \beta=0.5$ \\
\hline dimension & diameter & dimension & diameter \\
\hline \hline 1 & 34 & 1 & 23 \\
\hline 2 & 9 & 2 & 58 \\
\hline 3 & 5 & 3 & 18 \\
\hline 4 & 4 & 4 & 4 \\
\hline 5 & 2 & 5 & 5 \\
\hline
\end{tabular}

\section{Logarithmic Dimension Hypothesis}

In Chapter 2, we introduced the Logarithmic Dimension Hypothesis (or LDH), which states the dimension $m$ of an OSN satisfies

$$
m=O(\log N)
$$

where $N$ is the order of the OSN. For our datasets, we simplify this to

$$
m=\lceil\log N\rceil .
$$


We now explain how we attempted to validate the LDH by comparing real OSNs with the same order graphs generated by the GEO-P model with various dimensions, and find the most appropriate dimension.

Table 1 lists the spectral gaps of 10 YouTube samples of order around 5000. We take the average of the spectral gaps, giving a gap of 24.21. On the other hand, we simulated GEO-P model to generate the same order graphs from dimension 1 to 6 . Table 4 lists their spectral gaps. We can see that the best fit is between 19.2611 and 47.8424, which is for $m=3$ and $m=4$, respectively. Hence, we suggest that the OSNs of order 5000 have a dimension $\lceil 3.5\rceil=4$. It is consistent with the LDH, which demands a dimension of

$$
\lceil\log 5000\rceil=\lceil 3.69\rceil=4 .
$$

TABLE 4. Spectral gaps of graphs generated by the GEO-P model.

\begin{tabular}{|c||c|c|c|}
\hline \multicolumn{4}{|c|}{$N=5000, \alpha=0.7, \beta=0.15, p=1$} \\
\hline \hline dimension & $\lambda_{1}$ & $\lambda_{2}$ & gap \\
\hline 1 & 27.5004 & 27.3945 & 0.10593 \\
\hline 2 & 60.66 & 54.522 & 6.138 \\
\hline 3 & 82.9547 & 63.6936 & 19.2611 \\
\hline 4 & 134.5345 & 86.6921 & 47.8424 \\
\hline 5 & 221.943124 & 132.411872 & 89.531252 \\
\hline 6 & 379.201601 & 203.734353 & 175.467248 \\
\hline
\end{tabular}

Now we compare the diameters. Table 5 lists the 10 YouTube samples' diameters, we calculate the average diameter which is 7.8. Table 
6 lists the diameters of graphs generated by the GEO-P model, with dimension from 1 to 6 . We can see that the three-dimensional graph's diameter is close to the average diameter (with the average taken over all our samples); that is, 7.8. Hence, we have additional support for the LDH.

TABLE 5. Diameters of YouTube samples of order around 5000 .

\begin{tabular}{|c|c||c|c|}
\hline order & diameter & order & diameter \\
\hline 4497 & 8 & 3528 & 5 \\
\hline 5505 & 9 & 7507 & 8 \\
\hline 5503 & 11 & 6089 & 7 \\
\hline 6409 & 9 & 5053 & 6 \\
\hline 5897 & 7 & 6788 & 8 \\
\hline
\end{tabular}

TABLE 6. Diameters of graphs generated by the GEO-P model.

\begin{tabular}{|c||c|c|c|c|c|c|}
\hline \multicolumn{6}{|c|}{$N=5000, \alpha=0.7, \beta=0.15, p=1$} \\
\hline \hline dimension $(m)$ & 1 & 2 & 3 & 4 & 5 & 6 \\
\hline diameter & 51 & 10 & 5 & 4 & 2 & 2 \\
\hline
\end{tabular}

While our results are supportive of the $\mathrm{LDH}$, we do not claim to prove it. Larger samples would be necessary for that (ideally the entire network), or many more small samples. Further, there are many other graph properties we could check which is suggestive of two graphs being "similar" (although not necessarily isomorphic). We do speculate further on such similarity measures, but leave that for future work. 


\section{CHAPTER 4}

\section{New models for OSNs}

We described the GEO-P model in the previous chapter. In this chapter we simulate some new models based on the GEO-P model, analyse their properties, and check if they accurately fit OSN data. These new models are similar (but not identical to) to the GEO-P model, but use different ranking schemes such as age or degree.

Recall from Chapter 2 that we use $r(x, t)$ to indicate the rank of vertex $x$ at time-step $t$.

\section{Ranking by Age}

We first introduce the GEO-P(Age) model. In this model, an older vertex is more likely to attract more edges than a younger one. For a positive integer $t>0$, we define

$$
\operatorname{age}(x)=t
$$

where $t$ is the time-step when vertex $x$ is born.

The GEO-P(Age) model has four parameters: the attachment

strength $\alpha \in(0,1)$, the density parameter $\beta \in(0,1-\alpha)$, the dimension 
$m \in \mathbb{N}$, and the link probability $p \in(0,1]$. Let $S$ be unit hypercube in $\mathbb{R}^{m}$. The GEO-P(Age) model generates graphs via the following process.

1. At the initial time-step $t=0$, we choose $N$ vertices in $S$ at random, randomly assign each vertex a unique rank chosen from the set $[N]$, and set all vertices ages as 0 . An edge between a pair of vertices $x$ and $y$ is created with probability $p$ if

$$
d(x, y) \leq \max \{\operatorname{Rad}(x), \operatorname{Rad}(y)\} .
$$

2. At time-step $t>0$, a randomly chosen vertex $v \in V\left(G_{t-1}\right)$ dies. For the remaining vertices, if $r(x, t-1)>r(v, t-1)$, then we update their ranks by

$$
r(x, t)=r(x, t-1)-1,
$$

where $x \in V\left(G_{t-1}\right) \backslash\{v\}$. Hence, the rank of a vertex is determined by its age. Older vertices receive higher rank. On the other hand, a new vertex $u$ is born and is assigned rank $N$. We set its age as $t$. Then we add edges by the method described in Item 1 above.

The GEO-P(InvAge) model is similar to the GEO-P(Age) model. The difference is we assign new vertex higher rank as follows in item (2) below. 
1. At the initial time-step $t=0$, we choose $N$ vertices in $S$ at random, randomly assign each vertex a unique rank chosen from the set $[N]$, and set all vertices ages as 0 . An edge between a pair of vertices $x$ and $y$ is created with probability $p$ if

$$
d(x, y) \leq \max \{\operatorname{Rad}(x), \operatorname{Rad}(y)\} .
$$

2. At time-step $t>0$, a randomly chosen vertex $v \in V\left(G_{t-1}\right)$ dies, for remaining vertices, if $r(x, t-1)>r(v, t-1)$, then we update their ranks by

$$
r(x, t)=r(x, t-1)+1,
$$

where $x \in V\left(G_{t-1}\right) \backslash\{v\}$. Hence, a new vertex is more likely to be higher ranked. On the other hand, a new vertex $u$ is born and is assigned rank 1 . We set its age as $t$. Then we determine edges by the method described in Item (1) above.

Now we analyse degree distribution, diameter, spectral gap of graphs generated by the GEO-P(Age) and the GEO-P(InvAge) models. We applied the GEO-P(Age) and the GEO-P(InvAge) models to generated graphs embedded in $\mathbb{R}^{n}$, where $1 \leq n \leq 5$. Figures 4.1 and 4.2 display the degree distribution log-log plot. Tables 1 and 2 list the diameters and spectral gaps. 


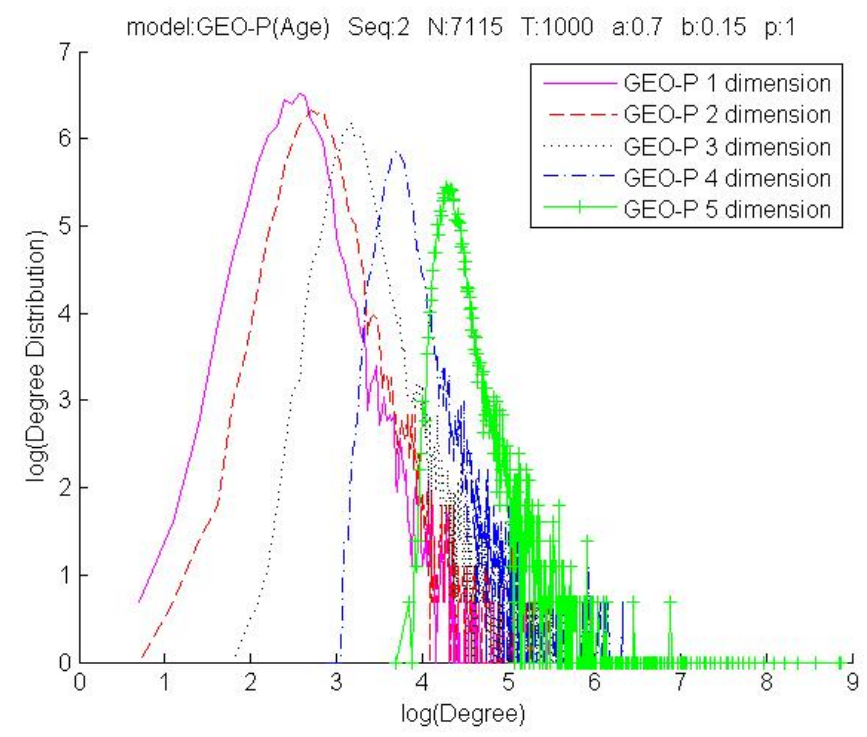

FiguRE 4.1. Degree distribution of a graph generated by the GEO-P(Age) model.

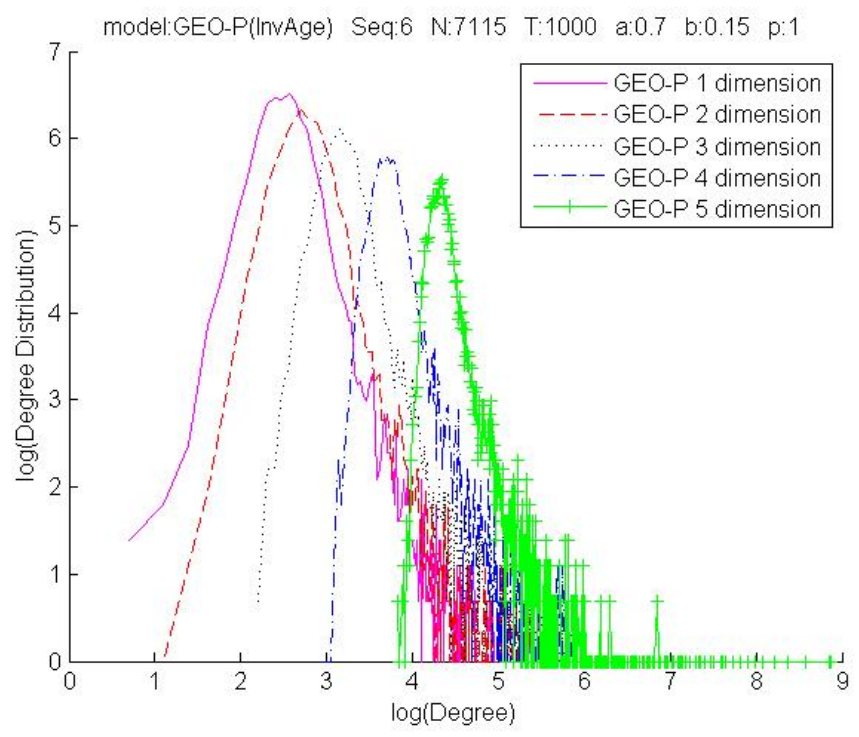

Figure 4.2. Degree distribution of a graph generated by the GEO-P(InvAge) model.

Comparing with the graphs generated by the GEO-P, GEO-P(Age), and GEO-P(InvAge) models, our results suggest that the models generate graphs with similar degree distributions; the diameters all decrease 
TABLE 1. Spectral gaps of graphs generated by the GEO-P(Age) and the GEO-P(InvAge) model.

\begin{tabular}{|c|c||c|c|}
\hline \multicolumn{4}{|c|}{$N=7115, \alpha=0.7, \beta=0.15, p=1$} \\
\hline \multicolumn{2}{|c|}{ GEO-P(Age) } & \multicolumn{2}{c|}{ GEO-P(InvAge) } \\
\hline dimension & gap & dimension & gap \\
\hline \hline 1 & 35.201192 & 1 & 0.913422 \\
\hline 2 & 32.509603 & 2 & 129.049955 \\
\hline 3 & 2.678022 & 3 & 32.13571 \\
\hline 4 & 52.84162 & 4 & 48.921797 \\
\hline 5 & 95.301035 & 5 & 96.451019 \\
\hline
\end{tabular}

TABLE 2. Diameters of graphs generated by the GEO$\mathrm{P}$ (Age) and the GEO-P(InvAge) model.

\begin{tabular}{|c|c||c|c|}
\hline \multicolumn{4}{|c|}{$N=7115, \alpha=0.7, \beta=0.15, p=1$} \\
\hline \multicolumn{2}{|c|}{ GEO-P(Age) } & GEO-P(InvAge) \\
\hline dimension & diameter & dimension & diameter \\
\hline \hline 1 & 33 & 1 & 61 \\
\hline 2 & 10 & 2 & 10 \\
\hline 3 & 5 & 3 & 5 \\
\hline 4 & 4 & 4 & 4 \\
\hline 5 & 3 & 5 & 2 \\
\hline
\end{tabular}

when the dimension increases; and the spectral gaps all decrease until the third dimension, then begin to increase. We may conclude that there is no significant difference between these models. In particular, all fit real OSNs well, and ranking by age does not provide a better fit to OSNs than the GEO-P model.

\section{Ranking by Degree}

The second group of OSN models we investigated are the GEO$\mathrm{P}(\mathrm{Deg})$ model and the GEO-P(GnpDeg) model. These two models 
have a new ranking system, where ranking is determined by the degrees of vertices. We assign larger degree vertex higher rank as it is plausible that more popular users in OSN may receive more invitations to become friends from other users. Hence, a higher degree vertex has a stronger ability of attracting new edges. If two vertices coincidentally have the same degree, then we take age as the second measurement to break ties.

The GEO-P(Deg) and the GEO-P(GnpDeg) models have four parameters: the attachment strength $\alpha \in(0,1)$, the density parameter $\beta \in(0,1-\alpha)$, the dimension $m \in \mathbb{N}$, and the link probability $p \in(0,1]$. Let $S$ be unit hypercube in $\mathbb{R}^{m}$, and $[N]$ be integers set from 1 to $N$. We describe the evolution process of the GEO-P(Deg) model:

1. At the initial time-step $t=0$, we choose $N$ vertices in $S$ at random, randomly assign each vertex a unique rank chosen from the set $[N]$, and set all vertices ages as 0 . An edge between a pair of vertices $x$ and $y$ is created with probability $p$ if

$$
d(x, y) \leq \max \{\operatorname{Rad}(x), \operatorname{Rad}(y)\} .
$$

We record degrees of all vertices at time-step $t=0$.

2. At time-step $t>0$, a randomly chosen vertex $v \in V\left(G_{t-1}\right)$ dies, we remove all edges connected to $v$. For remaining vertices, if $v x \in$ 
$E\left(G_{t-1}\right)$, then we update their degrees by

$$
\operatorname{deg}_{G_{t}}(x)=\operatorname{deg}_{G_{t-1}}(x)-1,
$$

where $x \in V\left(G_{t-1}\right) \backslash\{v\}$. On the other hand, a new vertex $u$ is born, we assign its age as $t$, and assign its degree and rank both 0. We now determine edges between every pair of vertices based on their ranks at time-step $t-1$. In the last step, we update and record vertices' ranks at time-step $t$ by as follows.

$$
\begin{aligned}
& K=\left|\left\{y \in V\left(G_{t}\right), \operatorname{deg}_{G_{t}}(y)>\operatorname{deg}_{G_{t}}(x)\right\}\right|, \\
& L=\mid\left\{y \in V\left(G_{t}\right), \operatorname{deg}_{G_{t}}(y)=\operatorname{deg}_{G_{t}}(x) \text { and age }(y)<\operatorname{age}(x)\right\} \mid, \\
& r(x, t)=K+L+1 .
\end{aligned}
$$

In (13), (14), and (15), $K$ indicates the number of vertices which degrees are bigger than vertex $x, L$ indicates the number of vertices which degree are equal to vertex $x$, but older than vertex $x$. Hence, $K+L$ is the number of vertices whose ranks are higher than vertex $x$. Note that only one vertex is born at every time-step $t$, so we will not have duplicated ranks.

The GEO-P(GnpDeg) model is similar to the GEO-P(Deg) model, the only difference is the GEO-P(GnpDeg) model starts from a random 
graph other than a GEO-P graph at time-step $t=0$. The GEO$\mathrm{P}($ GnpDeg) models generates graphs by following process:

1. At initial time-step $t=0$, we choose $N$ vertices in $S$ at random and generate a random graph $G(N, p)$. Thus, we determine edges between every pair of vertices by a fixed probability. Then we apply Formula 13, 14 and 15 to calculate vertices' ranks. Ages of all vertices are set to 0 .

2. At time-step $t>0$, a randomly chosen vertex $v \in V\left(G_{t-1}\right)$ dies, we remove all edges connected to $v$. For remaining vertices, if $v x \in$ $E\left(G_{t-1}\right)$, then we update their degrees by

$$
\operatorname{deg}_{G_{t}}(x)=\operatorname{deg}_{G_{t-1}}(x)-1,
$$

where $x \in V\left(G_{t-1}\right) \backslash\{v\}$. On the other hand, a new vertex $u$ is born, we assign its age as $t$, and assign its degree and rank both 0 . We now determine edges between every pair of vertices based on their ranks at time-step $t-1$. The last step, we update and record vertices' ranks at time-step $t$ by formulas (13), (14) and (15).

Note that in graphs generated by the GEO-P(GnpDeg) model, vertices ranks may not be unique, because vertices' ranks at time-step 
$t=0$ are determined by degrees, we may have some vertices with the same ranks and ages, which may cause a tie in following time-step $t$.

Now we analyse degree distribution, diameter, spectral gap of graphs generated by the GEO-P(Deg) and the GEO-P(GnpDeg) models. We applied the GEO-P(Deg) and the GEO-P(GnpDeg) models to generated graphs in dimensions 1 to 5 , and 1 to 4 , respectively. Figures 4.3 and 4.4 display the degree distribution log-log plot. Tables 4 and 3 list the diameters and gaps. We can see that the GEO-P(Deg) and the GEO-P(GnpDeg) models have different properties. Graphs generated by the GEO-P(Deg) model have similar properties as other GEO-Ptype models. But graphs generated by the GEO-P(GnpDeg) have some different properties. Their degree distribution does not follow a power law distribution, have a larger spectral gap comparing to other GEOP-type models, and their diameters do not shrink when the dimension increases. Their behaviors are closer to random graphs and this model is not a good one for OSNs. 


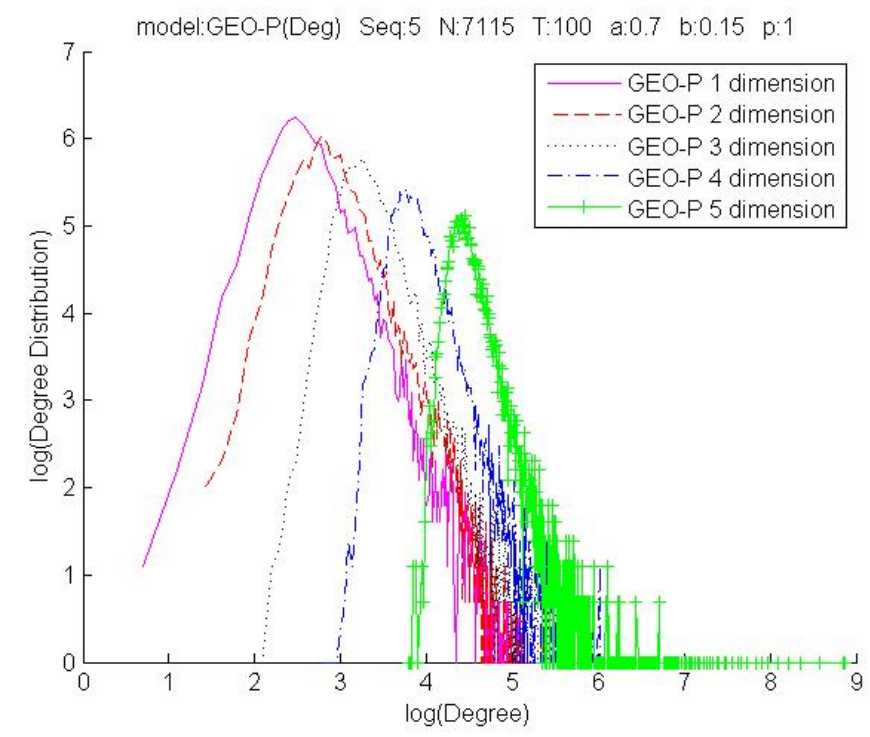

FiguRE 4.3. Degree distribution of graph generated by the GEO-P(Deg) model.

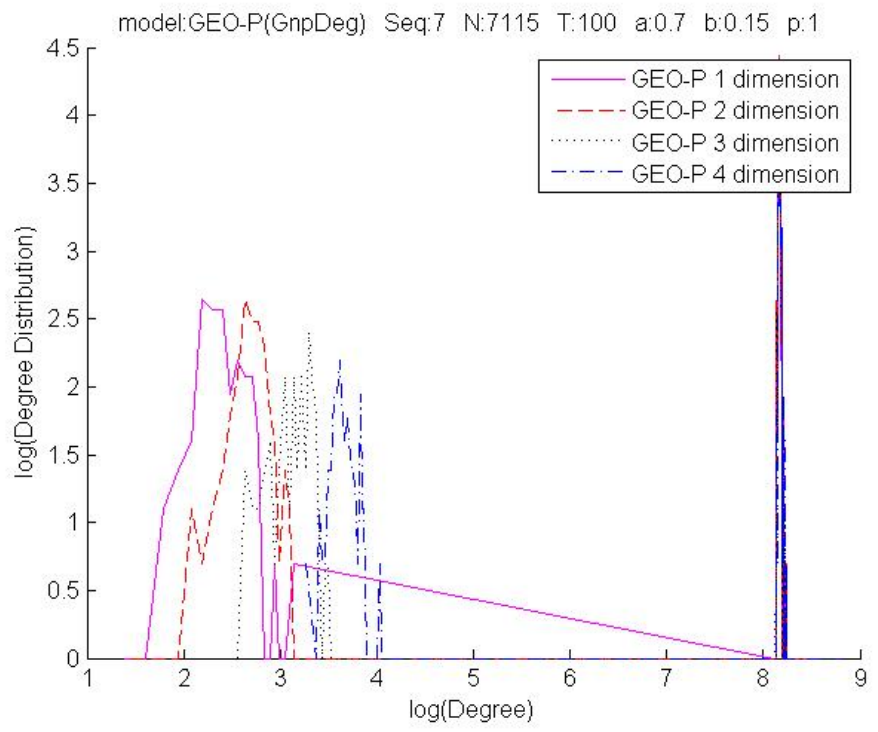

FiguRE 4.4. Degree distribution of graph generated by the GEO-P(GnpDeg) model. 
TABLE 3. Spectral gaps of graphs generated by the GEO-P(Deg) and the GEO-P(GnpDeg) model.

\begin{tabular}{|c|c||c|c|}
\hline \multicolumn{4}{|c|}{$N=7115, \alpha=0.7, \beta=0.15, p=1$} \\
\hline \multicolumn{3}{|c|}{ GEO-P(Deg) } & \multicolumn{2}{c|}{ GEO-P(GnpDeg) } \\
\hline dimension & gap & dimension & gap \\
\hline \hline 1 & 27.508783 & 1 & 3440.284205 \\
\hline 2 & 17.59291 & 2 & 3443.40596 \\
\hline 3 & 17.618218 & 3 & 3450.151086 \\
\hline 4 & 94.816041 & 4 & 3466.83475 \\
\hline 5 & 112.3931 & & \\
\hline
\end{tabular}

TABLE 4. Diameters of graphs generated by the GEO$\mathrm{P}(\mathrm{Deg})$ and the GEO-P(GnpDeg) model.

\begin{tabular}{|c|c||c|c|}
\hline \multicolumn{4}{|c|}{$N=7115, \alpha=0.7, \beta=0.15, p=1$} \\
\hline \multicolumn{2}{|c|}{ GEO-P(Deg) } & \multicolumn{2}{c|}{ GEO-P(GnpDeg) } \\
\hline dimension & diameter & dimension & diameter \\
\hline \hline 1 & 30 & 1 & 3 \\
\hline 2 & 9 & 2 & 3 \\
\hline 3 & 5 & 3 & 3 \\
\hline 4 & 4 & 4 & 3 \\
\hline 5 & 2 & & \\
\hline
\end{tabular}

\section{Tension Parameter}

Figure 4.5 shows the influence regions of vertices 5,7 and 16 . We see that vertices 7 and 16 both fall in the influence region of vertex 5. Based on the GEO-P model, the pairs of vertices 5, 7 and 5, 16 may both obtain edges (let us assume the link probability parameter $p=1$ ). But on the other hand, we see that vertex 7 has a larger influence region then vertex 16, and correspondingly vertex 7 should have stronger ability of attracting edges. Hence, the overall ability of 
obtaining edge of vertices 5 and 7 should be stronger than vertices 5 and 16 .

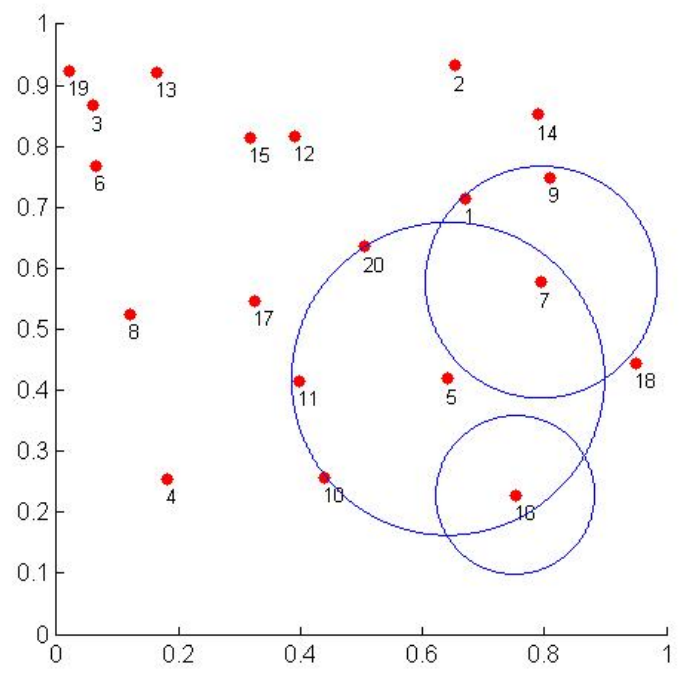

FiguRE 4.5. Influence region of vertices 5, 7 and 16 .

We introduce tension parameter $h \in(-\infty, 0)$. Recall that the influence region is determined by the rank of vertex; that is, a higher rank vertex has stronger ability of obtaining edges than lower rank vertex. We define the tension probability, written

$$
T P(x, t)=r(x, t)^{h},
$$


where $x$ is a given vertex, and $r(x, t)$ is the rank of vertex $x$ at time-step $t$. Note that $r(x, t) \geq 1$, so $T P(x, t) \in(0,1)$. We use

$$
\frac{T P(x, t)+T P(y, t)}{2}
$$

to donate the overall tension probability of vertices $x$ and $y$.

Based on the tension parameter, we introduce the GEO-P(Ten) model. This model has four parameters: the attachment strength $\alpha \in$ $(0,1)$, the density parameter $\beta \in(0,1-\alpha)$, the dimension $m \in \mathbb{N}$, and the tension parameter $h \in(-\infty, 0)$. The GEO-P(Ten) model is the same as the GEO-P model, except for how to determine edges between every pair of vertices. Let $S$ be unit hypercube in $\mathbb{R}^{m}$, and $[N]$ be integers set from 1 to $N$. The GEO-P(Ten) model generates graphs by following process:

1. At the initial time-step $t=0$, we randomly choose $N$ vertices in $S$, randomly assign vertex unique rank chosen from set $[N]$. An edge between pair of vertices $x$ and $y$ is created, if

$$
d(x, y) \leq \max (\operatorname{Rad}(x), \operatorname{Rad}(y))\left(\frac{T P(x, t)+T P(y, t)}{2}\right)
$$

2. At time-step $t>0$, a randomly chosen vertex $v \in V\left(G_{t-1}\right)$ dies, a new vertex $u$ is born and is assign a rank randomly chosen from set 
$[N]$. We update ranks of vertices $x \in V\left(G_{t-1}\right) \cap V\left(G_{t}\right)$ by (2), then evaluate edges by 16

Now we analyse degree distribution, diameter, spectral gap of graphs generated by the GEO-P(Ten) model. We applied the GEO-P(Ten) model to generated graphs in dimensions 1 to 5 inclusive, and we choose different values for tension parameter: $h=-0.1, h=-0.3$ and $h=-0.7$. Figures 4.6, 4.7 and 4.8 display the degree distribution log-log plots. Tables 6 and 5 list the diameters and gaps.

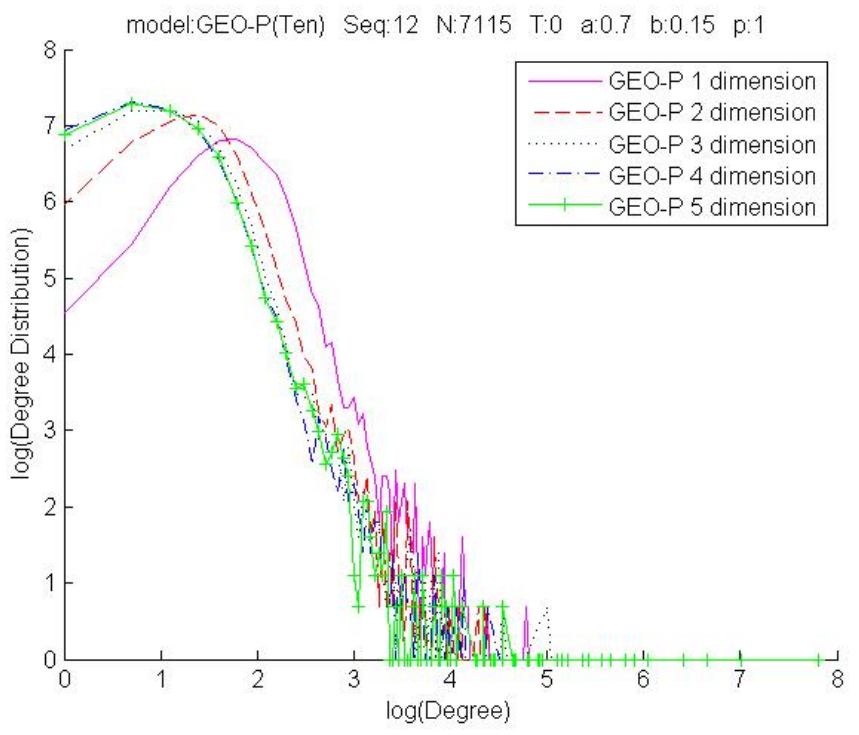

FiguRE 4.6. Degree distribution of graph generated by the GEO-P(Ten) model, with tension parameter $h=$ -0.1 .

We can see that graphs generated by the GEO-P(Ten) model have small spectral gaps and diameters, which are almost of the same order of the GEO-P model. From Figures 4.6, 4.7, and 4.8, we can see 


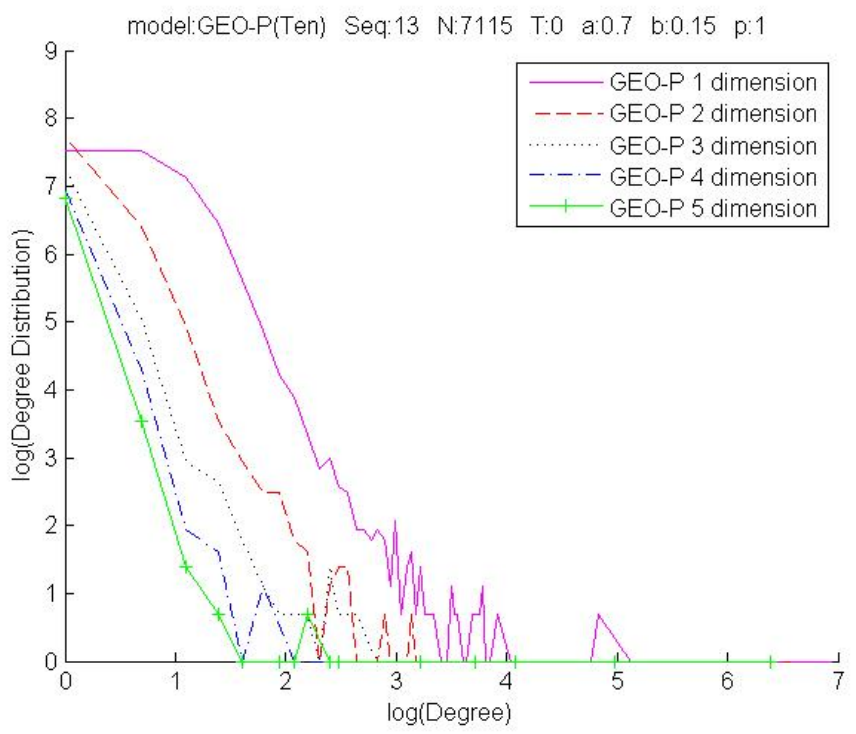

FiguRE 4.7. Degree distribution of graph generated by the GEO-P(Ten) model, with tension parameter $h=$ -0.3 .

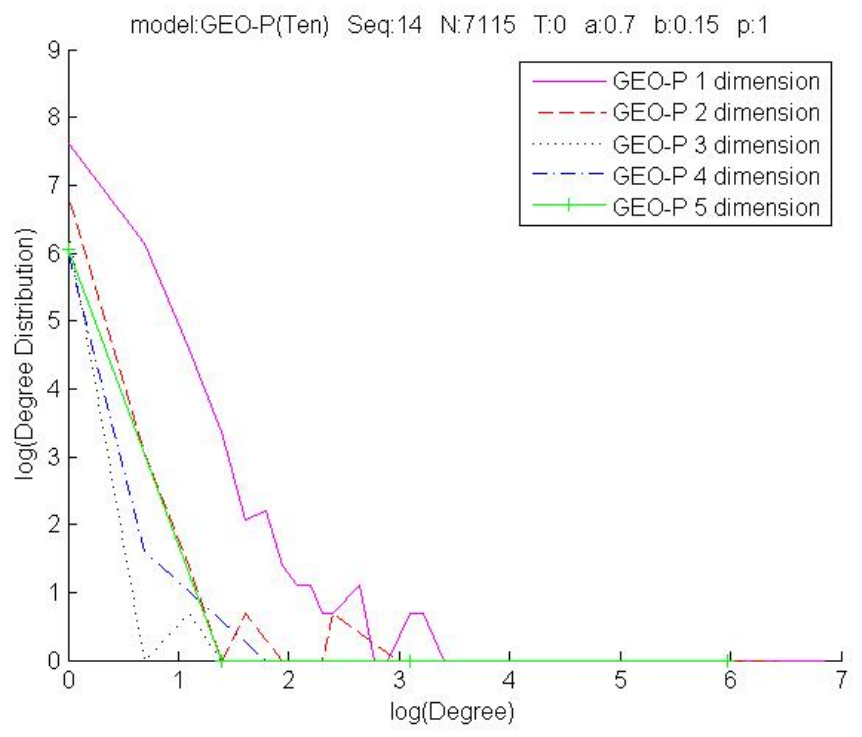

FiguRE 4.8. Degree distribution of graph generated by the GEO-P(Ten) model, with tension parameter $h=$ -0.7 . 
TABLE 5. Spectral gaps of graphs generated by the GEO-P(Ten) model, in dimensions 1 to 5 inclusive.

\begin{tabular}{|c|c||c|c||c|c|}
\hline \multicolumn{2}{|c|}{ GEO-P(Ten) $N=7115, \alpha=0.7, \beta=0.15, p=1$} \\
\hline \multicolumn{2}{|c|}{$h=-0.1$} & \multicolumn{2}{c|}{$h=-0.3$} & \multicolumn{2}{c|}{$h=-0.7$} \\
\hline $\operatorname{dim}$ & gap & $\operatorname{dim}$ & gap & dim & gap \\
\hline \hline 1 & 14.170589 & 1 & 9.048678 & 1 & 13.048242 \\
\hline 2 & 0 & 2 & 9.286437 & 2 & 12.406163 \\
\hline 3 & 11.410871 & 3 & 10.354512 & 3 & 13.150765 \\
\hline 4 & 15.54475 & 4 & 10.186001 & 4 & 14.226928 \\
\hline 5 & 20.845548 & 5 & 12.422439 & 5 & 15.133812 \\
\hline
\end{tabular}

TABLE 6. Diameters of graphs generated by the GEO$\mathrm{P}(\mathrm{Ten})$ model.

\begin{tabular}{|c||c|c||c|c||c|c|}
\hline \multicolumn{6}{|c|}{ GEO-P(Ten) $N=7115, \alpha=0.7, \beta=0.15, p=1$} \\
\hline \multicolumn{2}{|c||}{$h=-0.1$} & \multicolumn{2}{|c|}{$h=-0.3$} & \multicolumn{2}{|c|}{$h=-0.7$} \\
\hline dimension & diam & $V(C)$ & diam & $V(C)$ & diam & $V(C) \mid$ \\
\hline \hline 1 & 20 & 7098 & 6 & 6300 & 4 & 2673 \\
\hline 2 & 20 & 6953 & 7 & 3149 & 4 & 990 \\
\hline 3 & 20 & 6847 & 7 & 1648 & 3 & 571 \\
\hline 4 & 15 & 6744 & 6 & 1152 & 3 & 415 \\
\hline 5 & 12 & 6747 & 5 & 977 & 2 & 428 \\
\hline
\end{tabular}

that graphs generated by the GEO-P(Ten) model follow a power law degree distribution in a larger range than the GEO-P model. Our initial results indicate that the GEO-P(Ten) model accurately fits OSN data as well as the GEO-P model. 


\section{CHAPTER 5}

\section{Conclusion and Open Problems}

We introduced OSNs and verified graph theoretic properties of OSNs. Our computational results provide support for observed properties of OSNs: namely, power law degree distributions, the small world property, and bad spectral expansion.

The simulation results in sections 3.1 and 3.3 support the Logarithmic Dimension Hypothesis, which conjectures that the dimension of OSNs is

$$
m=\lceil\log N\rceil
$$

where $N$ is the order of the network. We compared the spectral gap of OSN samples and graphs generated by the GEO-P model in different dimensions, and our results suggest that the best fitting dimension is about $\log N$. We also compared the diameter of OSN samples and graphs generated by the GEO-P model, and found this was best fit to real OSN data when the dimension was about $\log N$.

We also introduced variants of GEO-P-type models which naturally simulate OSNs. We used the models to simulate OSNs, and verify how they fit OSNs by calculating the three main properties. Table 1 
summarizes results from our simulations. According to that table, most of the GEO-P-type models fit OSNs well, and the degree distribution of graphs generated by the GEO-P(Ten) model follow a power law degree distribution for a bigger range of vertices. Only the GEO-P(GnpDeg) model does not fit OSNs well because of it does not seem to generate power law graphs.

TABLE 1. The GEO-P-type models simulation and calculation results

\begin{tabular}{|c|c|c|c|}
\hline model & power law & small wold & spectral gap \\
\hline GEO-P & yes & yes & small \\
\hline GEO-P(Age) & yes & yes & small \\
\hline GEO-P(InvAge) & yes & yes & small \\
\hline GEO-P(Deg) & yes & yes & small \\
\hline GEO-P(GnpDeg) & no & yes & large \\
\hline GEO-P(Ten) & yes & yes & small \\
\hline
\end{tabular}

Several open problems arose while this research was conducted. We hope to be able to address these problems in future work. We collect and state these problems below.

1. OSNs usually have large orders, with many millions of vertices and edges. Owing to computational bottlenecks, we chose to work with smaller samples of OSNs, with orders in the thousands of vertices and edges. Higher order samples or even a whole OSN sample will likely give a more accurate result. 
2. In Chapters 3 and 4, we only evaluate diameters of graphs to verify the small word property. The clustering coefficient is also a measurement of the small world property. Calculating clustering coefficients is another step to verify the small world property in both the models and the sampled OSN data. Tuning the models to give the correct clustering coefficient may give more evidence for the LDH.

3. For the GEO-P model, we have a few parameters, such as: the attachment strength, the density parameter, the dimension, and the link probability. How do we best choose these parameters?

4. In our OSN models, we use consecutive integers to assign vertices ranks, which leads to similar influence regions at different time-step t. Assigning vertices with positive real number as ranks may be more natural. One idea is that vertices ranks could be determined based on their popularity. We calculate the degree ratio for every vertex, which is defined as

$$
R A(x, t)=\frac{\operatorname{deg}_{G_{t}}(x)}{\sum_{v \in V\left(G_{t}\right)} \operatorname{deg}_{G_{t}}(v)}
$$

Hence, the degree ratio indicates how popular the corresponding user is; that is, a bigger degree ratio refers to a more popular user. Note that since $R A(x, t) \in[0,1)$, we can then define rank function 
based on the degree ratio. For example, we may define

$$
R(x, T)=R A(x, t)^{k},
$$

where $k$ is a negative real number.

5. For the GEO-P Model, rigorous proofs were given that the model a.a.s. generates graphs with a power law degree distribution, low diameter, high clustering coefficient, and bad spectral expansion. See Chapter 2, Theorems 2.1, 2.2, 2.3, 2.4, and 2.5 for the rigorous results for the GEO-P model. Can we rigorously prove with probability tending to 1 as $n$ and $t$ tend to infinity, that the models for OSNs presented in this thesis satisfy the desired properties?

6. From our simulation results, the GEO-P(GnpDeg) model does not fit the real OSN data well. However, we may need to run the time $t$ much longer for the model to generate graphs with the desired properties. 


\section{CHAPTER 6}

\section{Appendix}

The following original code of mysql database and matlab was used to simulate OSNs and the various stochastic models presented in this thesis. Further, we provide the code used to mine the real OSN data for various properties.

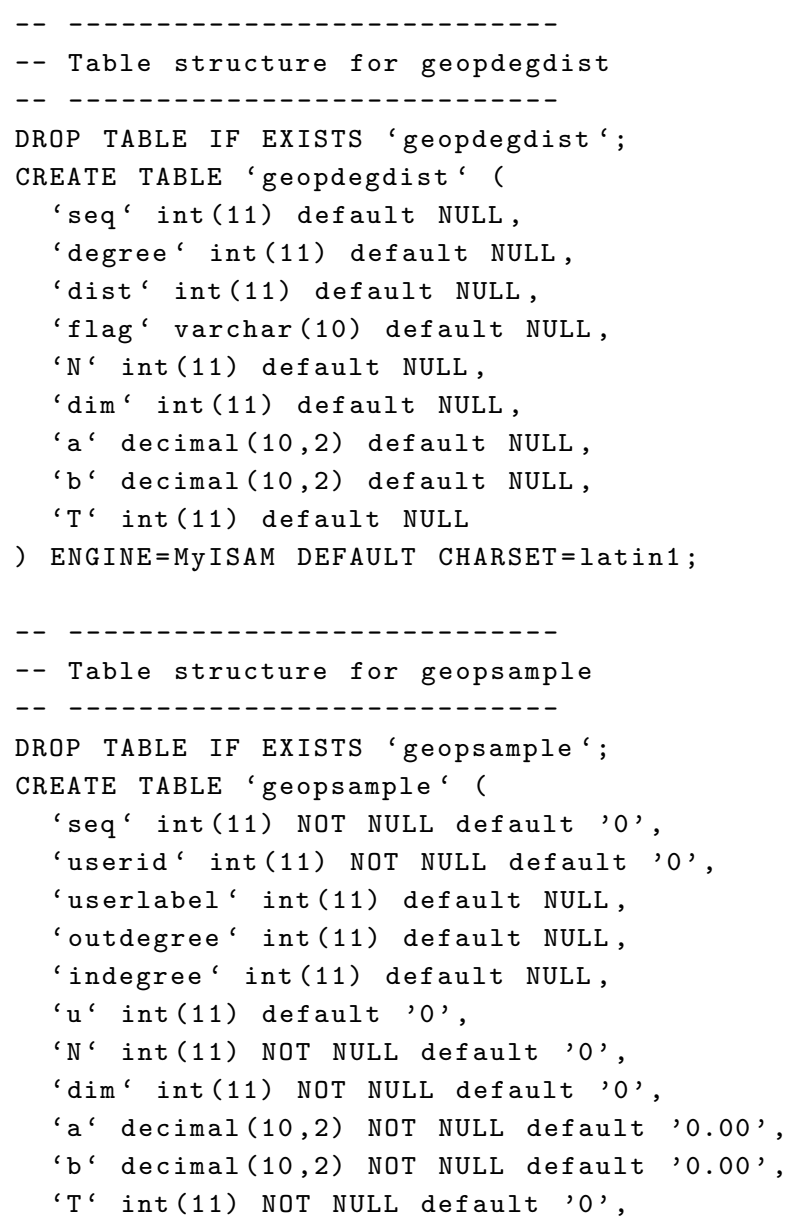




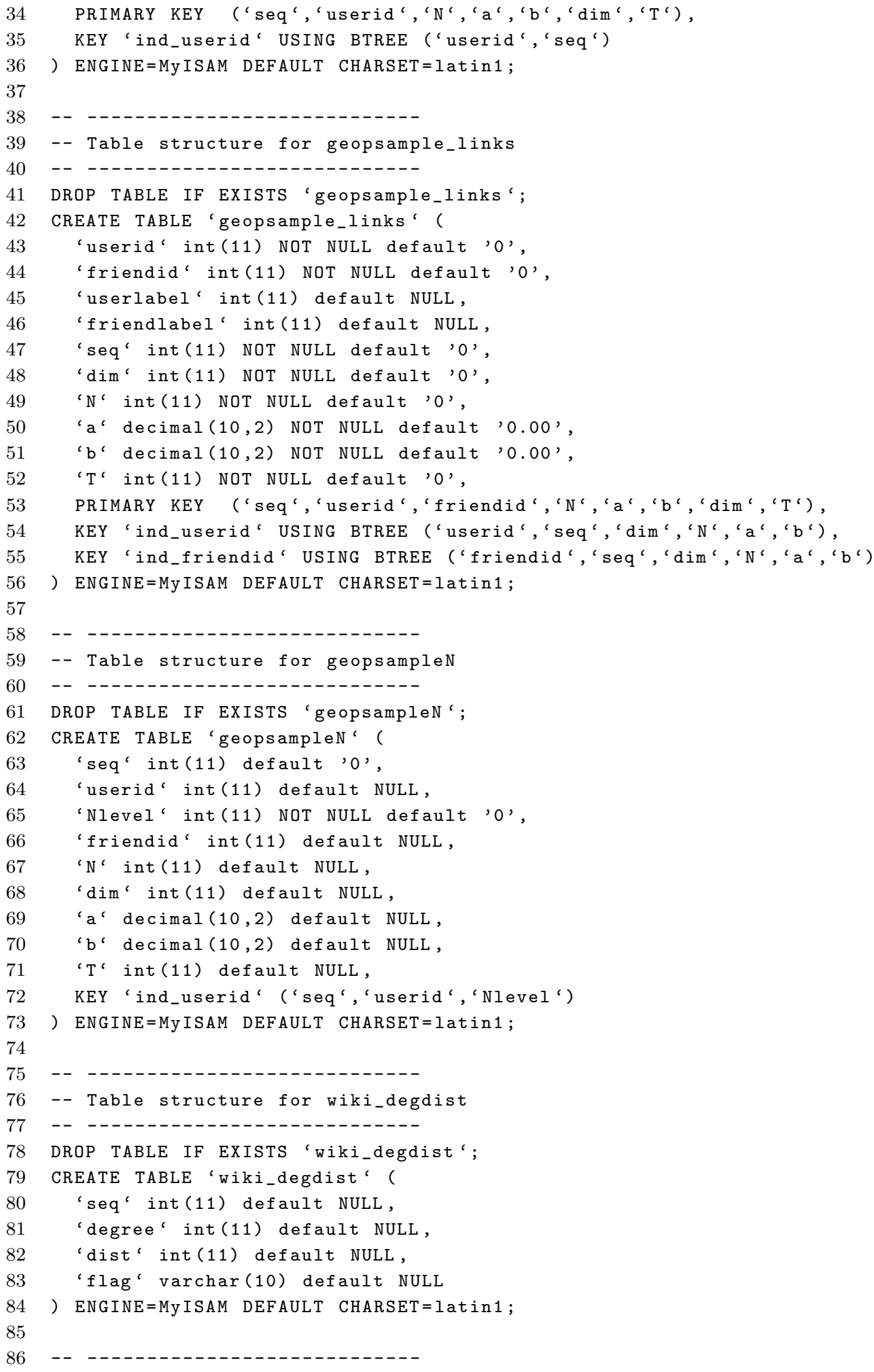




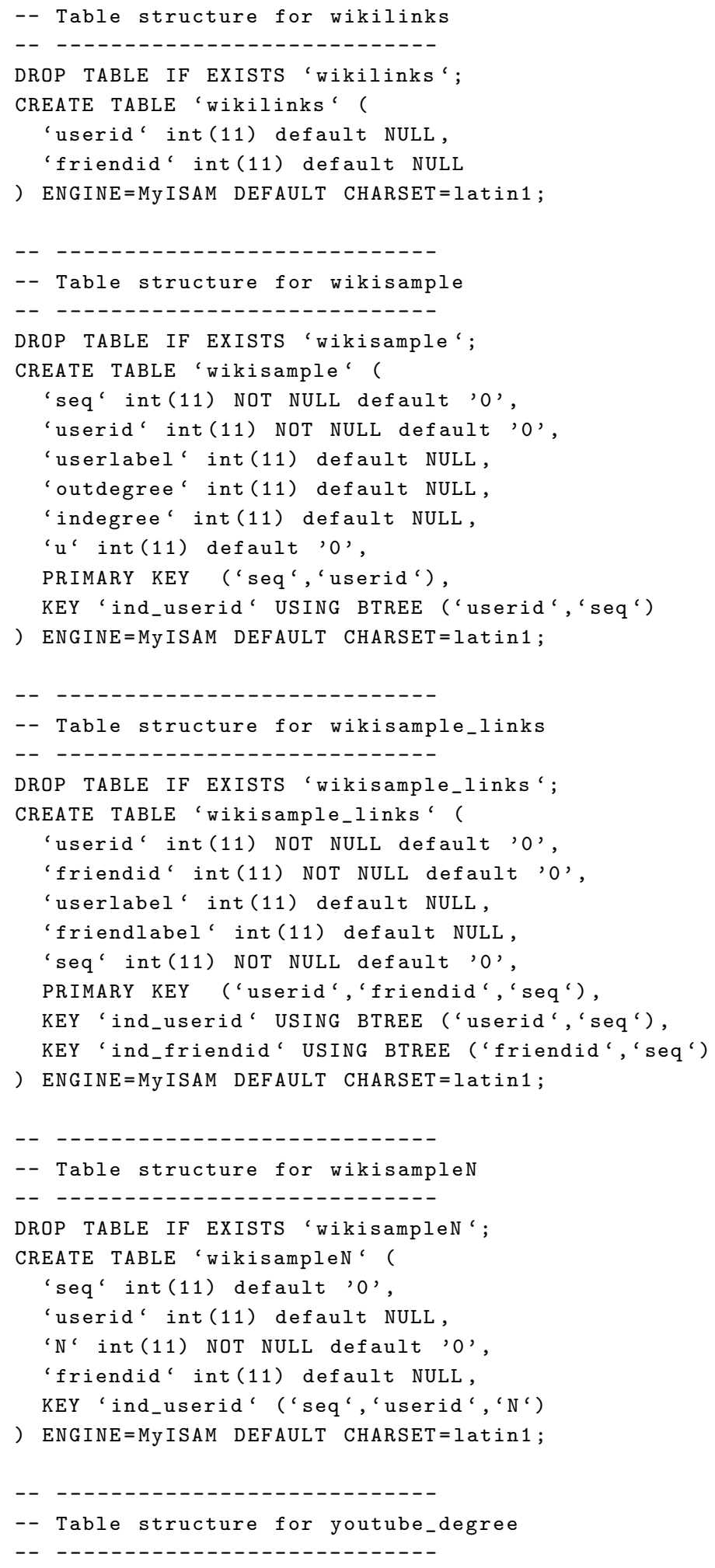




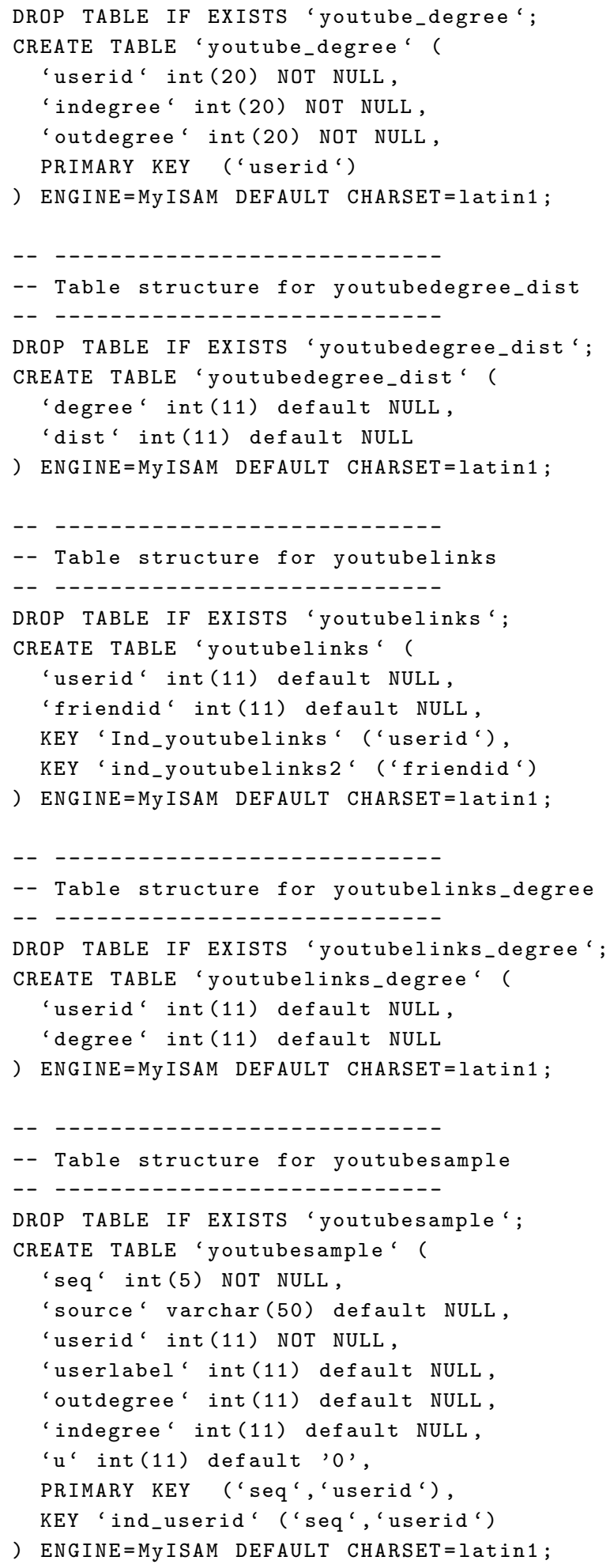




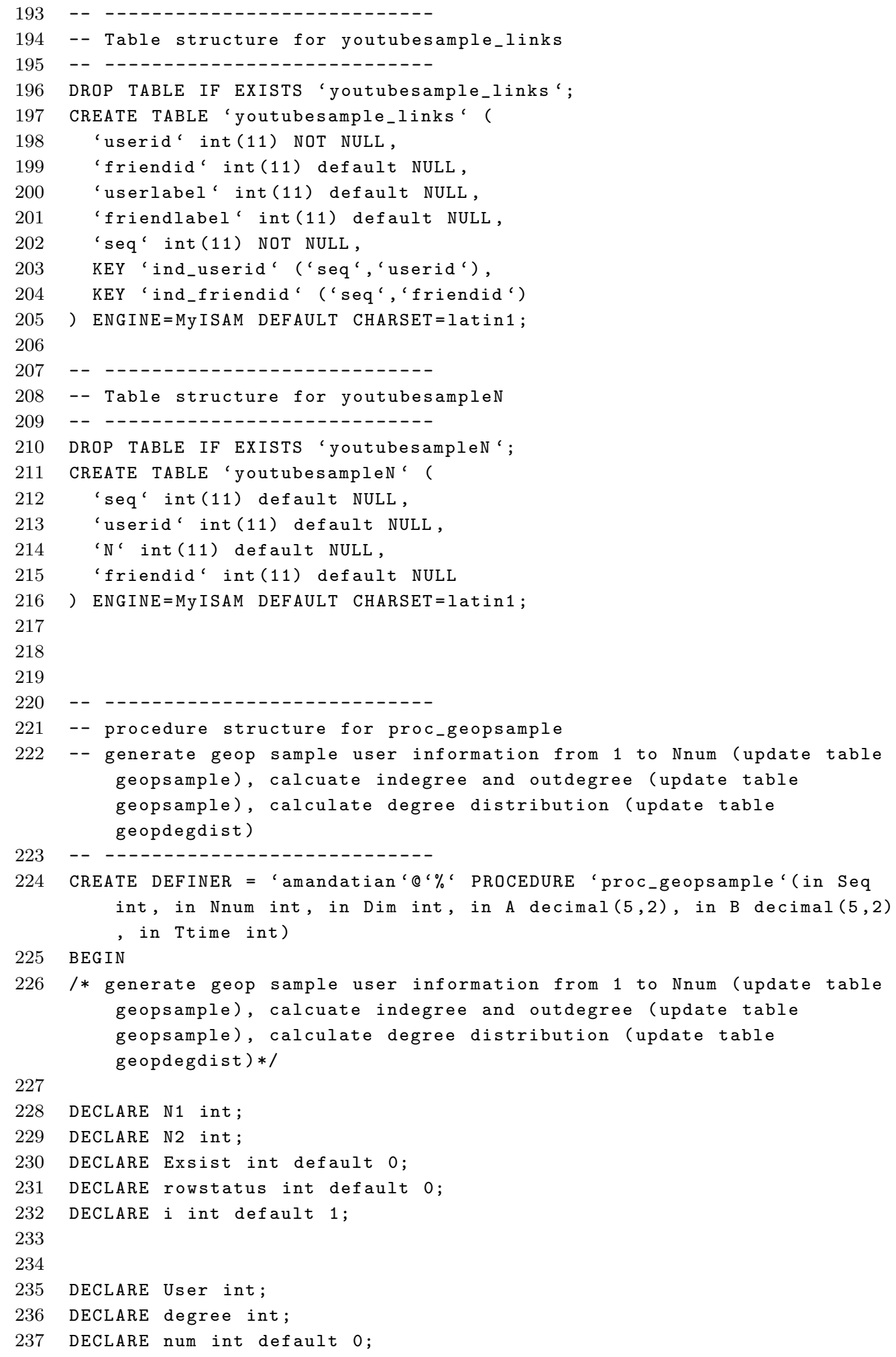




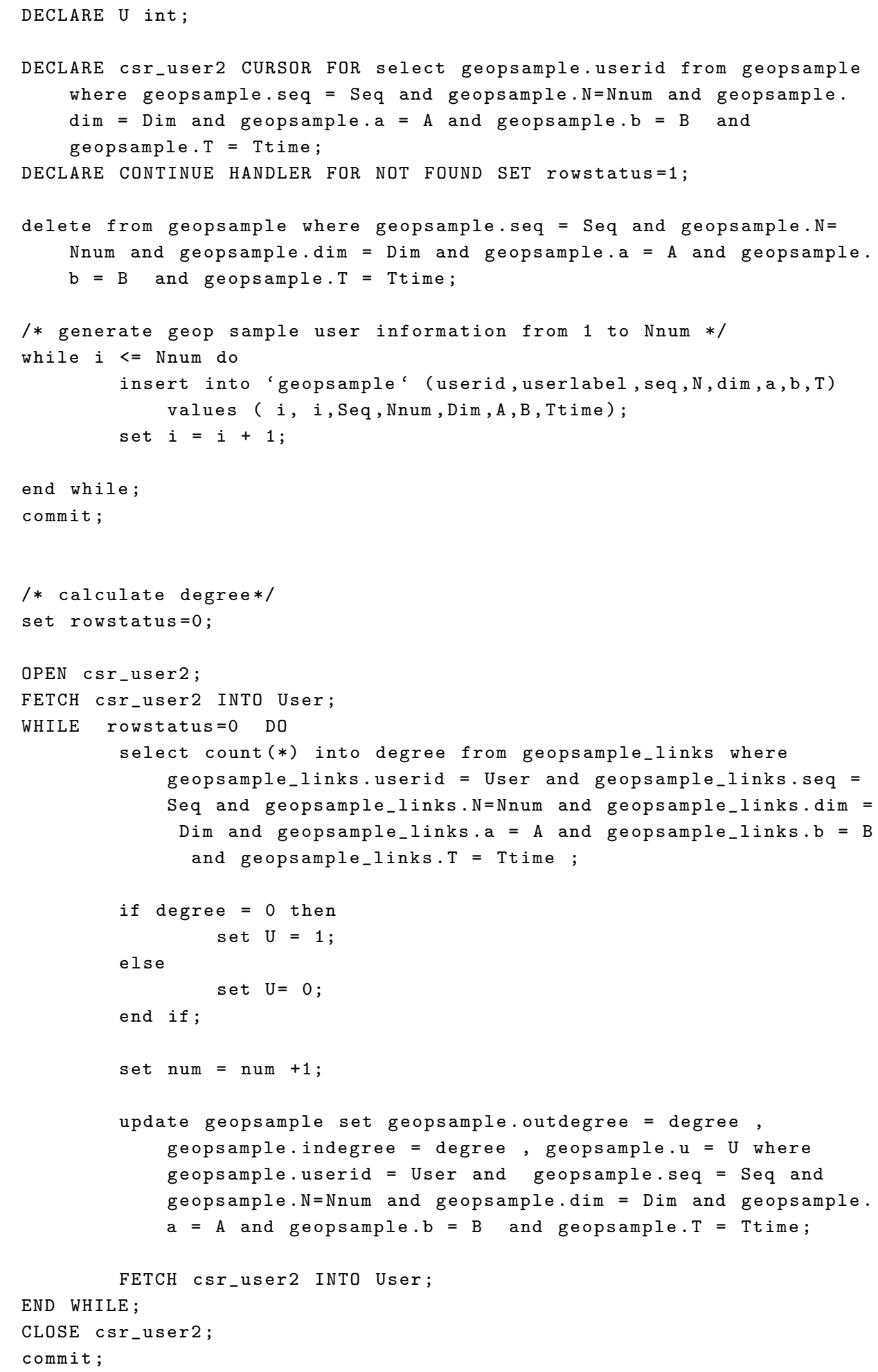

DECLARE csr-user 2 CURSOR FOR select geopsample.userid from geopsample where geopsample.seq = Seq and geopsample.N=Nnum and geopsample. dim $=$ Dim and geopsample.a $=A$ and geopsample.b $=B$ and geopsample. T = Ttime; 


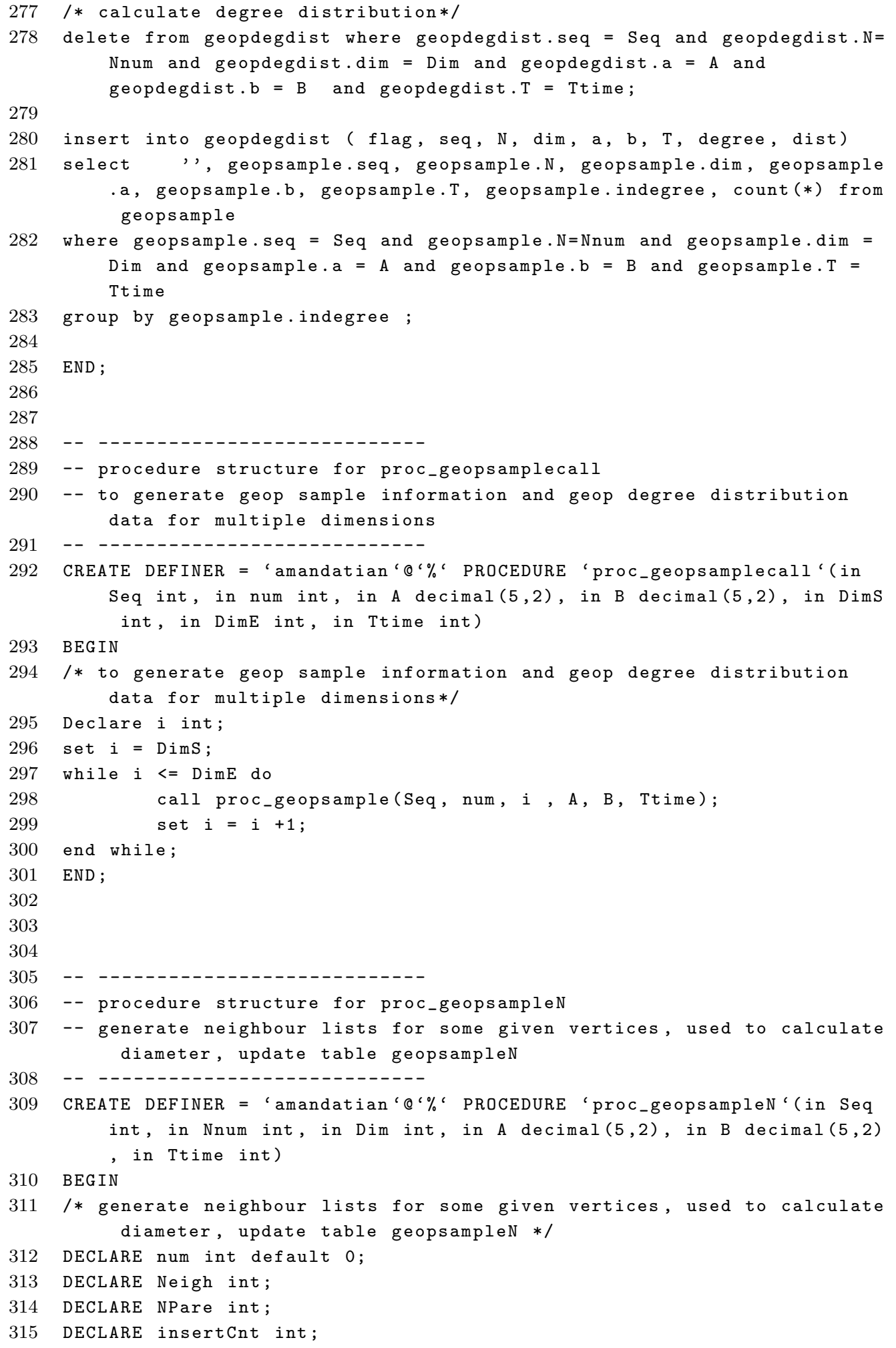




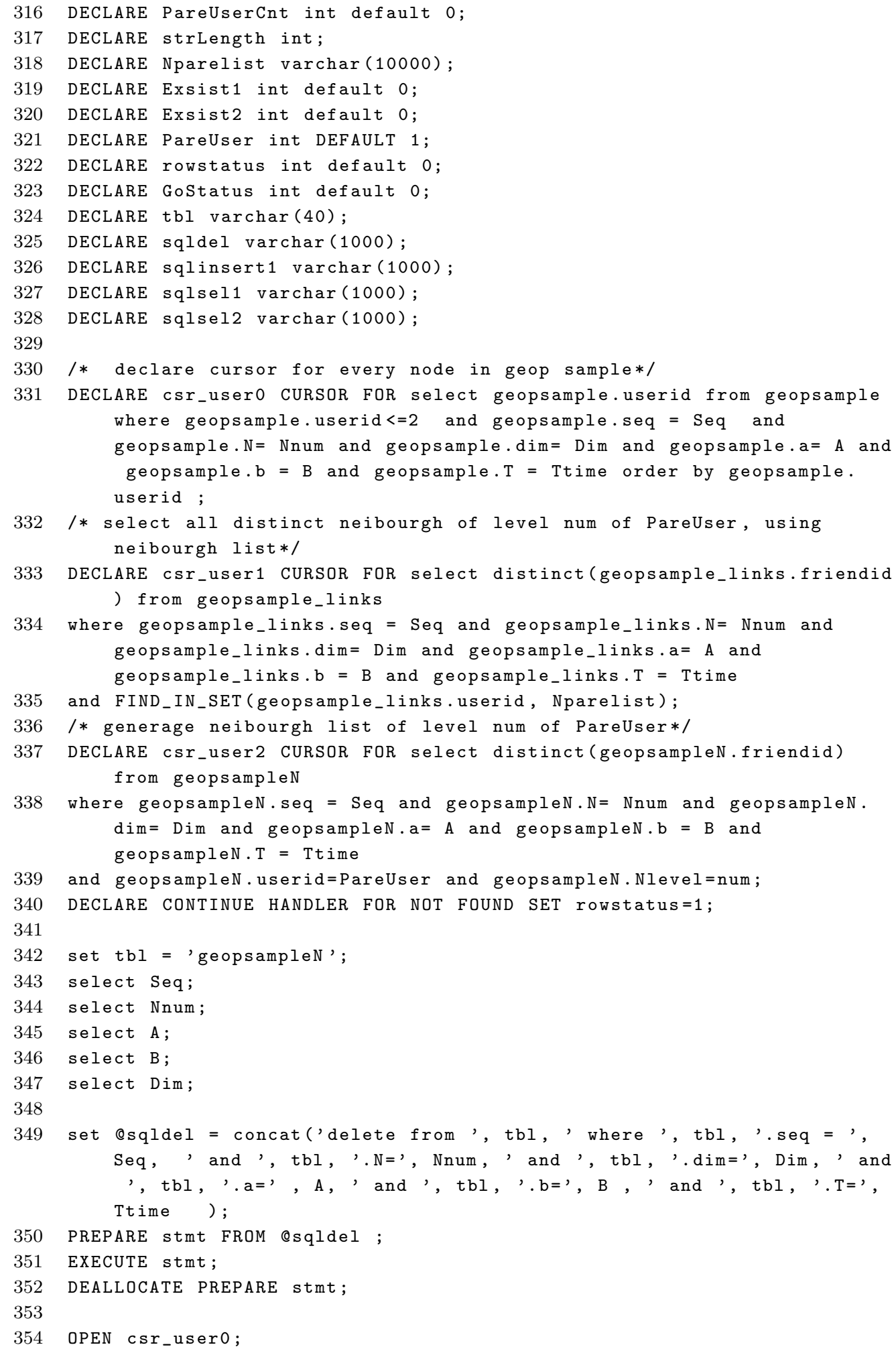




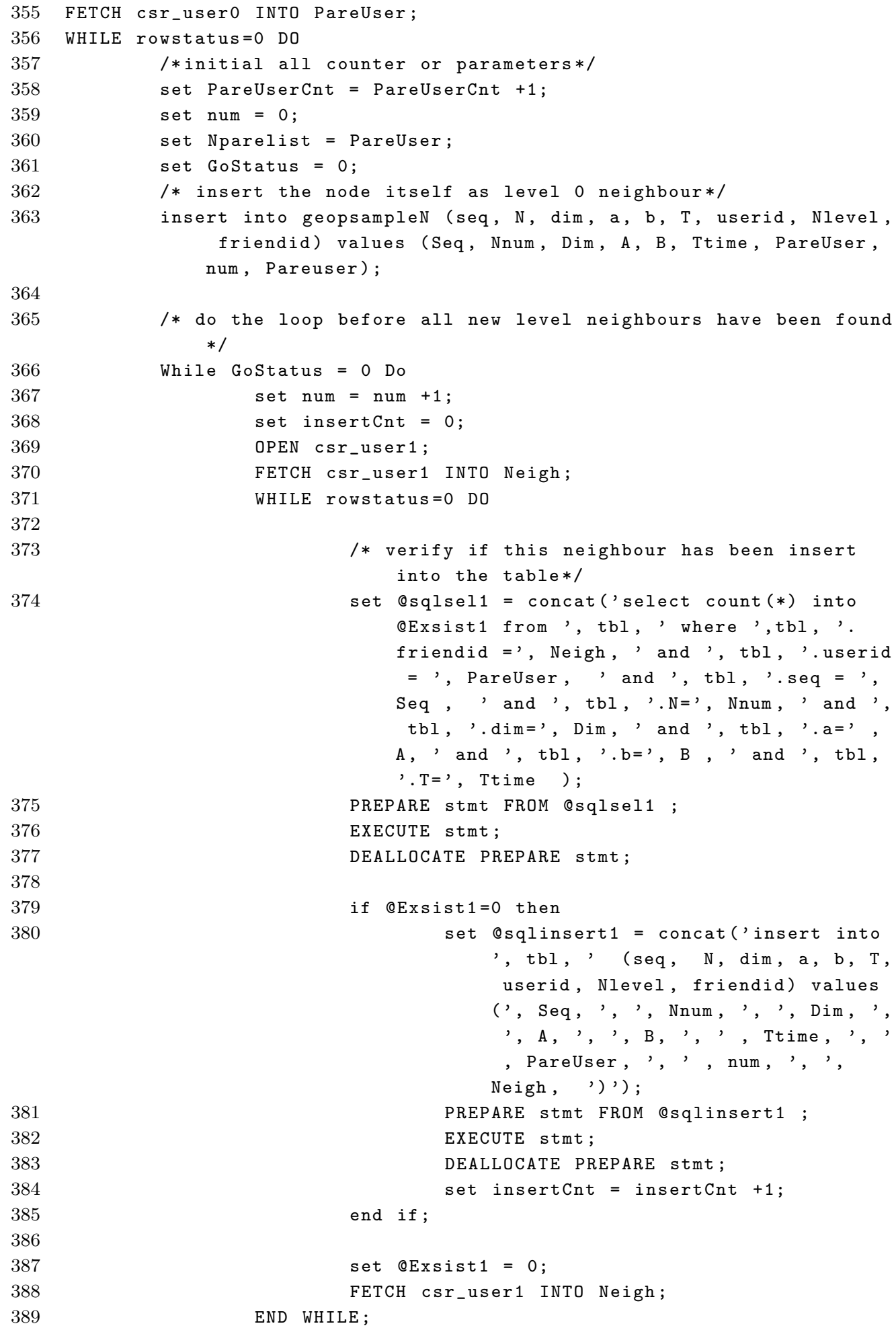

/*initial all counter or parameters $*$ / set PareUserCnt $=$ PareUserCnt +1 ;

set num = 0 ;

set Nparelist $=$ PareUser;

set GoStatus = 0 ;

/* insert the node itself as level 0 neighbour*/ insert into geopsampleN (seq, N, dim, a, b, T, userid, Nlevel, friendid) values (Seq, Nnum, Dim, A, B, Ttime, PareUser, num, Pareuser);

/* do the loop before all new level neighbours have been found $* /$

While GoStatus $=0$ Do

set num = num +1 ;

set insertCnt $=0$;

OPEN csr_user1;

FETCH csr_user1 INTO Neigh;

WHILE rowstatus=0 DO

/* verify if this neighbour has been insert into the table*/

set Qsqlsel1 = concat ('select $\operatorname{count}(*)$ into @Exsist1 from ', tbl, , where',tbl, '. friendid =', Neigh, ', and ', tbl, '.userid $=$, PareUser, , and ', tbl, , seq =, , Seq, ' and ', tbl, '. $N=$, , Nnum, ' and ', tbl, '.dim=, Dim, , and ', tbl, '. $a=$, , $A$, and ', tbl,, $\mathrm{b}=$, , B, , and ', tbl, '. $\mathrm{T}=$ ', Ttime );

PREPARE stmt FROM Qsqlsel1; EXECUTE stmt; DEALLOCATE PREPARE stmt ;

if $Q$ Exsist $1=0$ then set Qsqlinsert1 = concat ('insert into , tbl, (seq, $\mathrm{N}, \mathrm{dim}, \mathrm{a}, \mathrm{b}, \mathrm{T}$, userid, Nlevel, friendid) values (', Seq, ', ', Nnum, ', ', Dim, ', ', A, ', ', B, ', , , Ttime, , , , PareUser, ', , num, ', ', Neigh, ') ');

PREPARE stmt FROM @sqlinsert1 ; EXECUTE stmt; DEALLOCATE PREPARE stmt; set insertCnt $=$ insertCnt +1 ; end if;

set QExsist $1=0$; FETCH csr_user1 INTO Neigh; END WHILE 


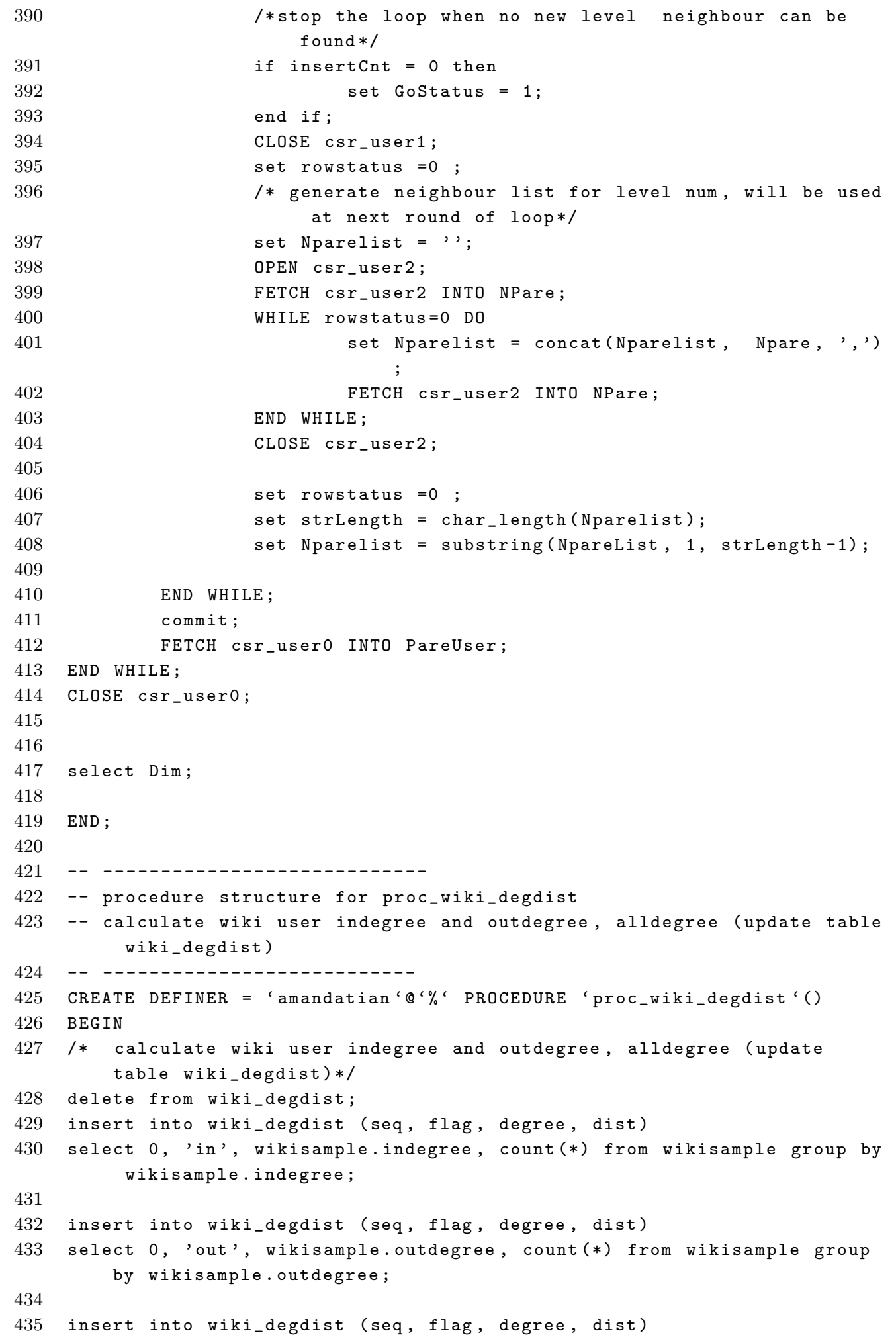

424

431

434 


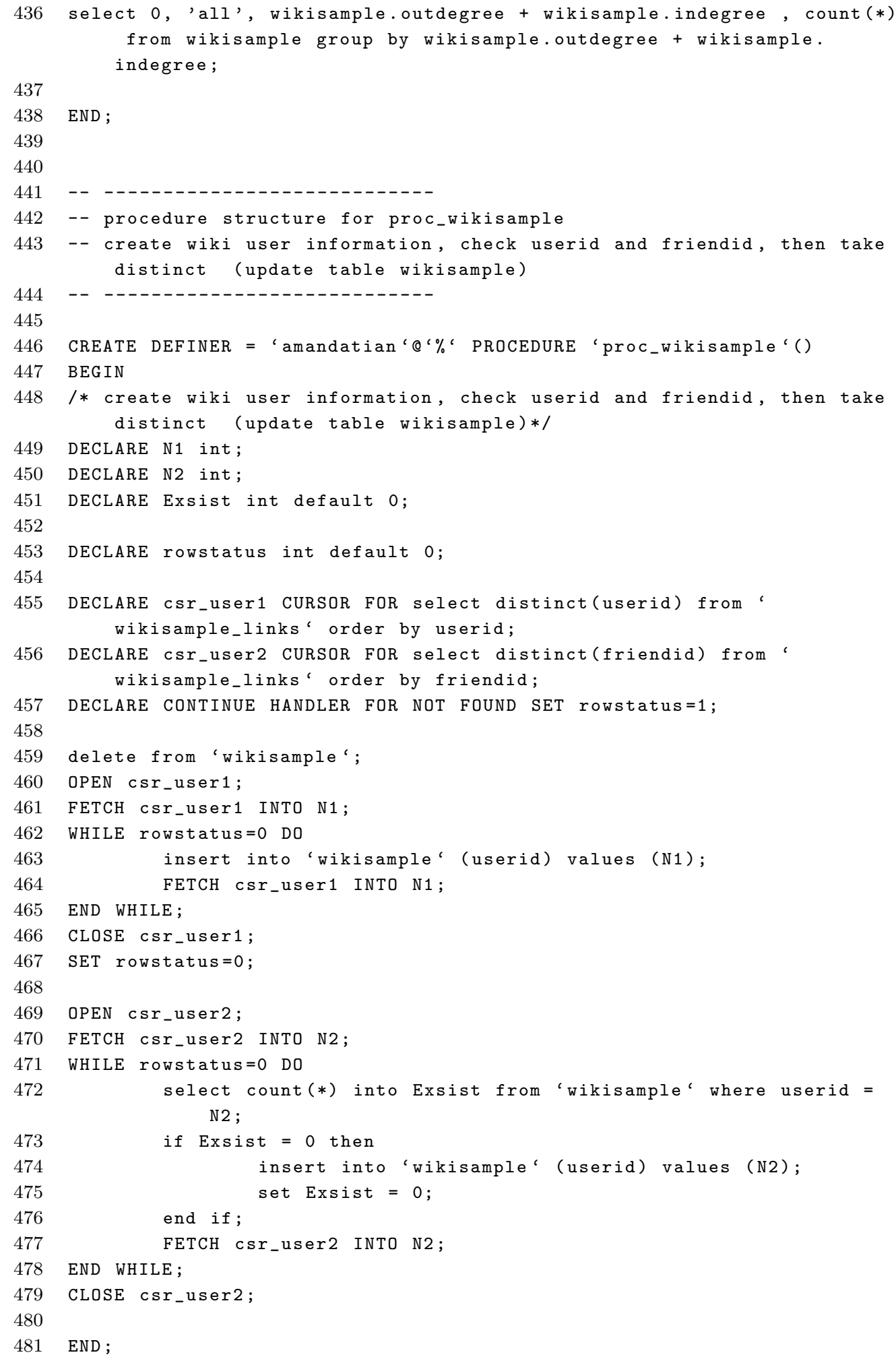




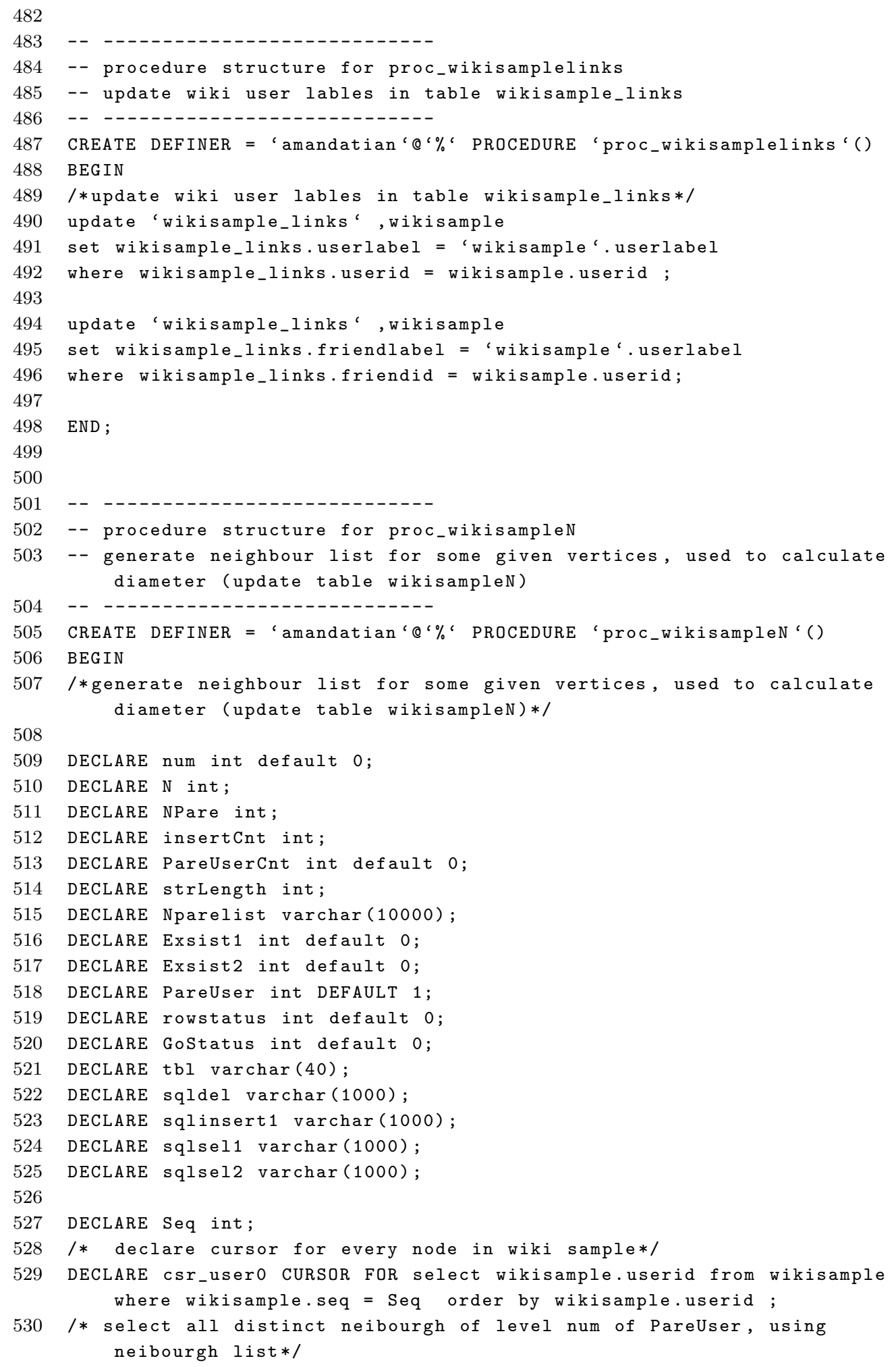


536

539

540

541

542

544

545

546

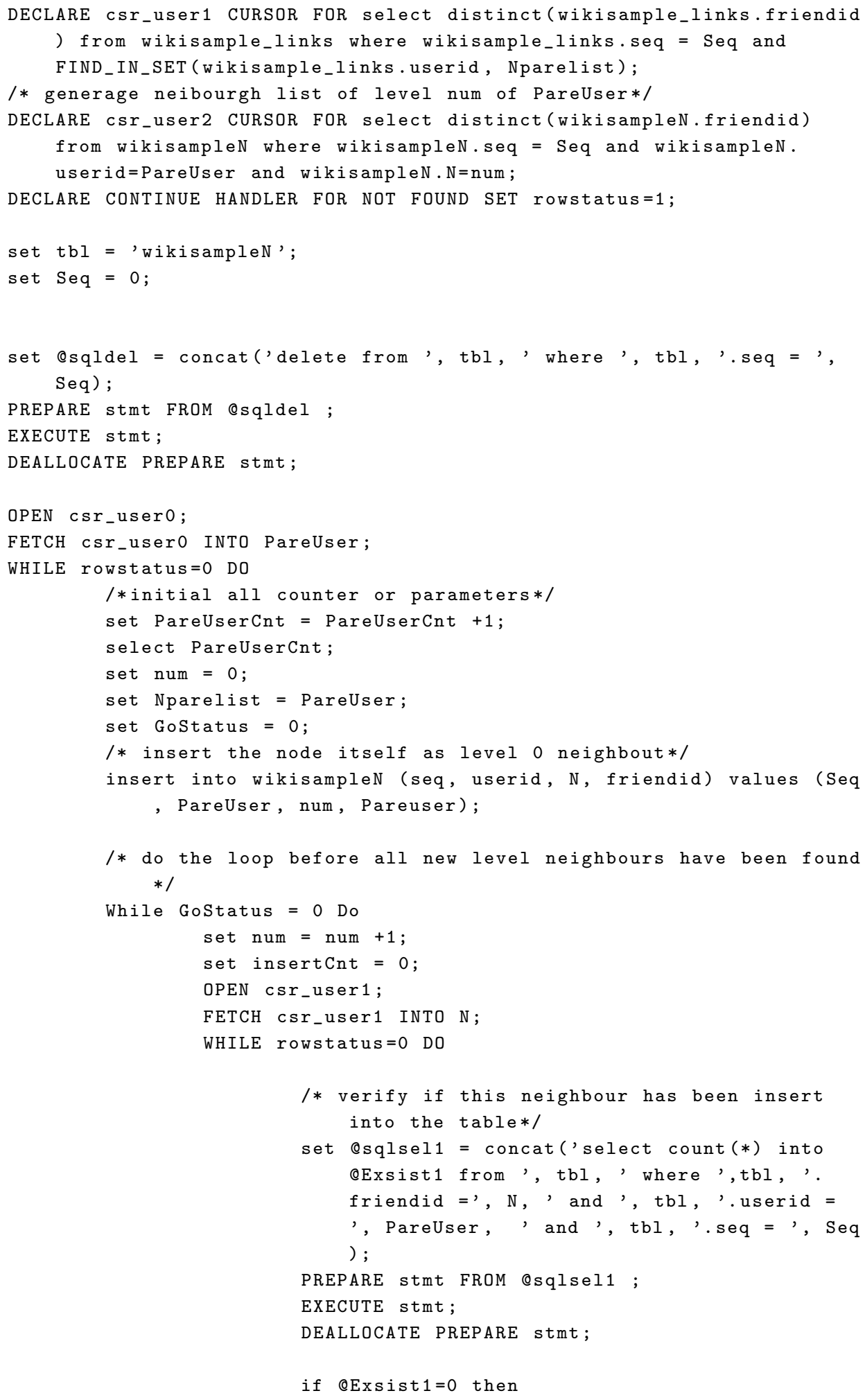




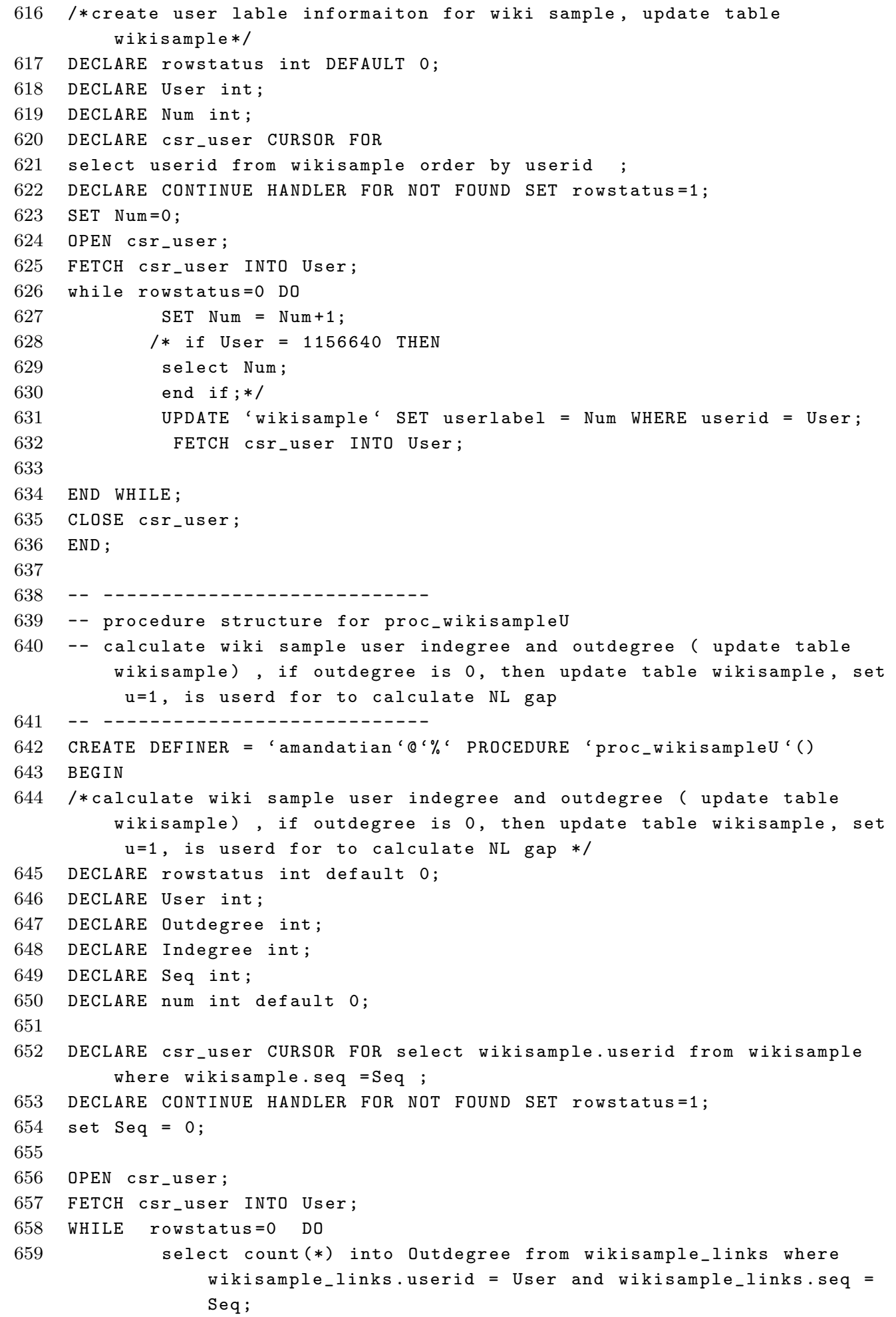




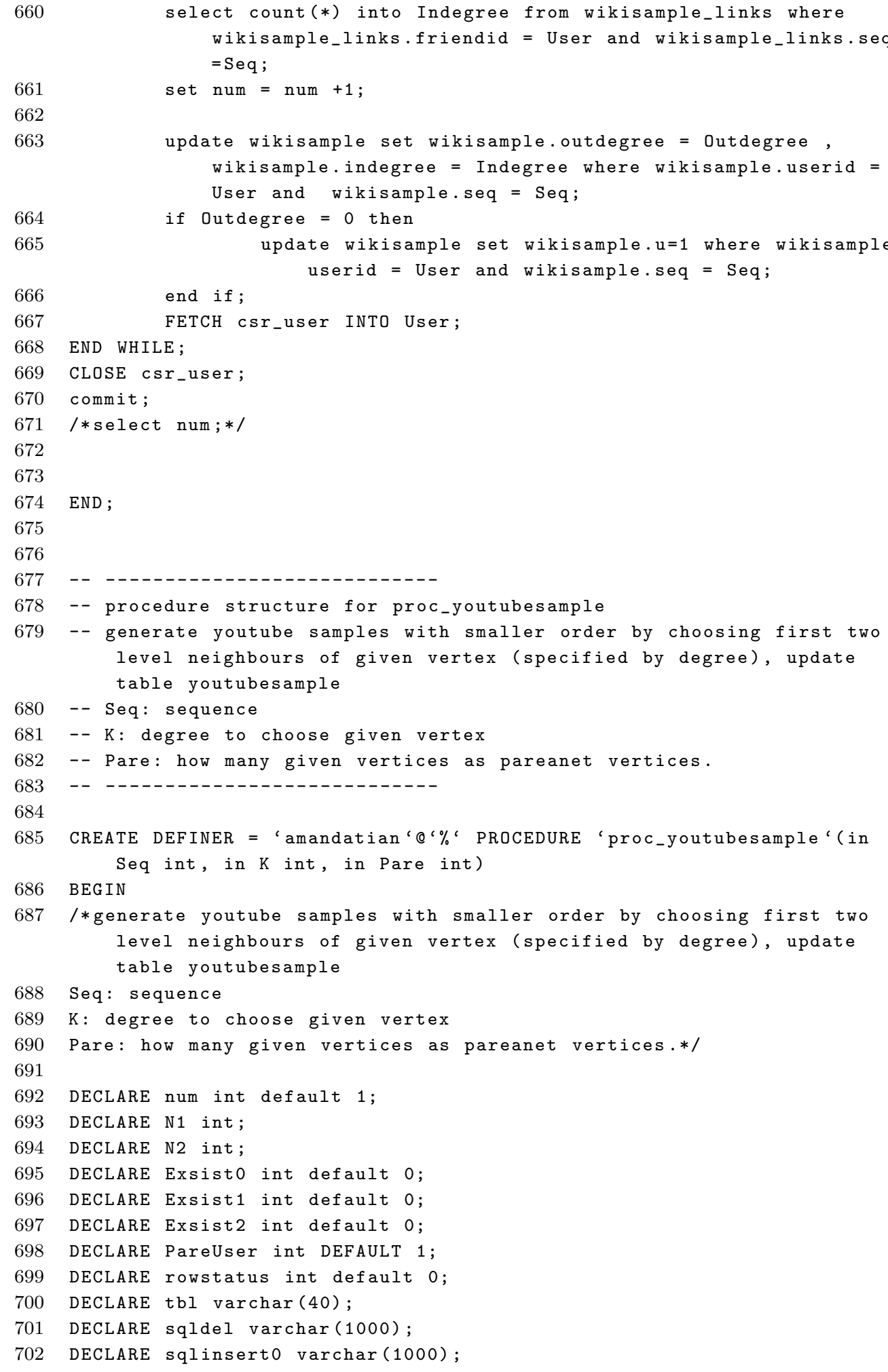

661

662

663

664

665

666

667

668

669

670

671

672

673

674

675

676

677

678

679

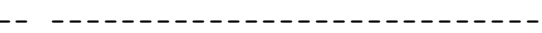

-- procedure structure for proc_youtubesample

-- generate youtube samples with smaller order by choosing first two level neighbours of given vertex (specified by degree), update table youtubesample

680 -- Seq: sequence

681 -- K: degree to choose given vertex

682 -- Pare: how many given vertices as pareanet vertices.

683

684

685

686 


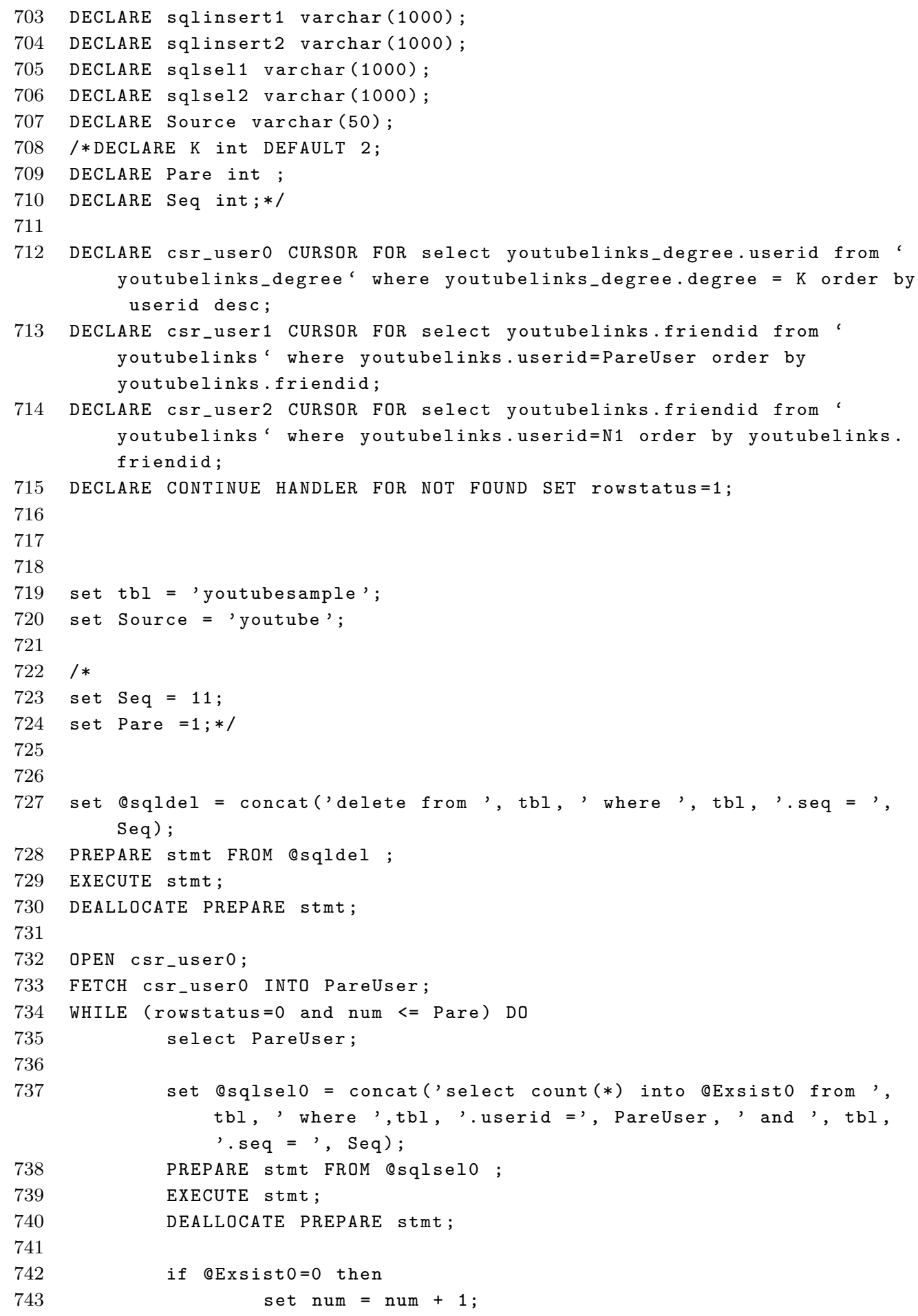




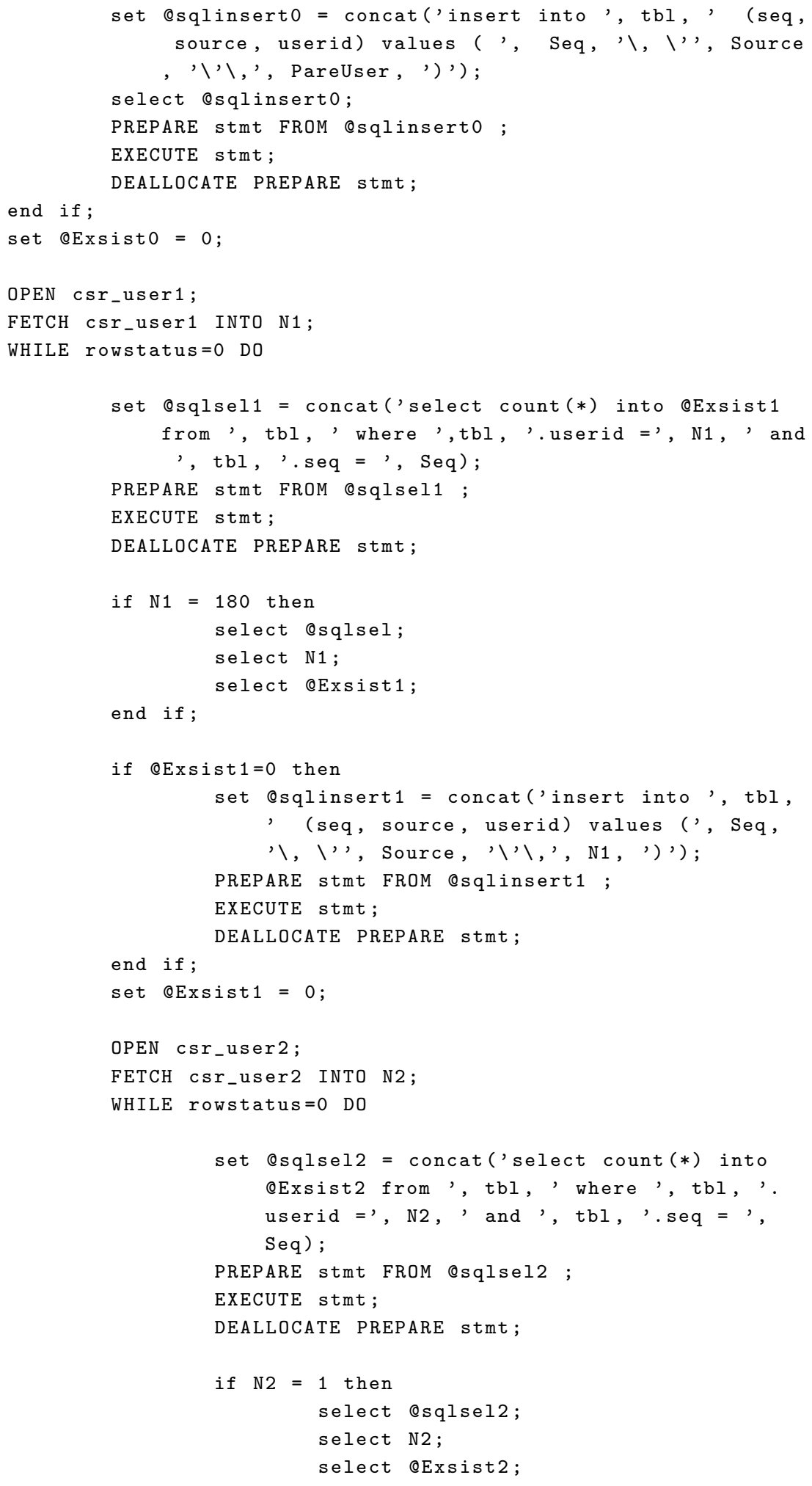


end if;

if @Exsist2 $=0$ then

set $@$ sqlinsert 2 = concat ('insert into

', tbl, ' (seq, source, userid)

values (', Seq, '\, \', Source, '(', ', N2, ')');

PREPARE stmt FROM @sqlinsert2;

EXECUTE stmt;

DEALLOCATE PREPARE stmt;

end if;

set @Exist $2=0$;

FETCH csr_user2 INTO N2;

END WHILE;

CLOSE csr_user2;

set rowstatus $=0$;

FETCH Csr_user1 INTO N1;

END WHILE;

CLOSE csr_user 1 ;

set rowstatus $=0$;

FETCH Csr_usero IntO PareUser;

END WHILE;

CLOSE csr_usero;

commit;

END ;

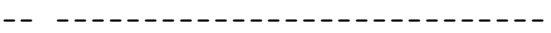

-- procedure structure for proc_youtubeSample0rder

-- update youtube sample user label, update table youtubesample -- --------------------------

CREATE DEFINER = 'amandatian' $@$ '\%" PROCEDURE 'proc_youtubeSampleOrder'( in Seq int)

BEGIN

/*update youtube sample user label, update table youtubesample*/

DECLARE rowstatus int DEFAULT 0 ;

DECLARE User int;

DECLARE Num int;

DECLARE tbl varchar $(40)$;

/*DECLARE Seq int; */

DECLARE csr_user CURSOR FOR select youtubesample.userid from

youtubesample where youtubesample.seq = Seq order by youtubesample .userid ;

DECLARE CONTINUE HANDLER FOR NOT FOUND SET rowstatus=1;

SET Num $=0$;

/*SET Seq $=11 ; * /$

OPEN csr_user ;

FETCH csr_user INTO User; 


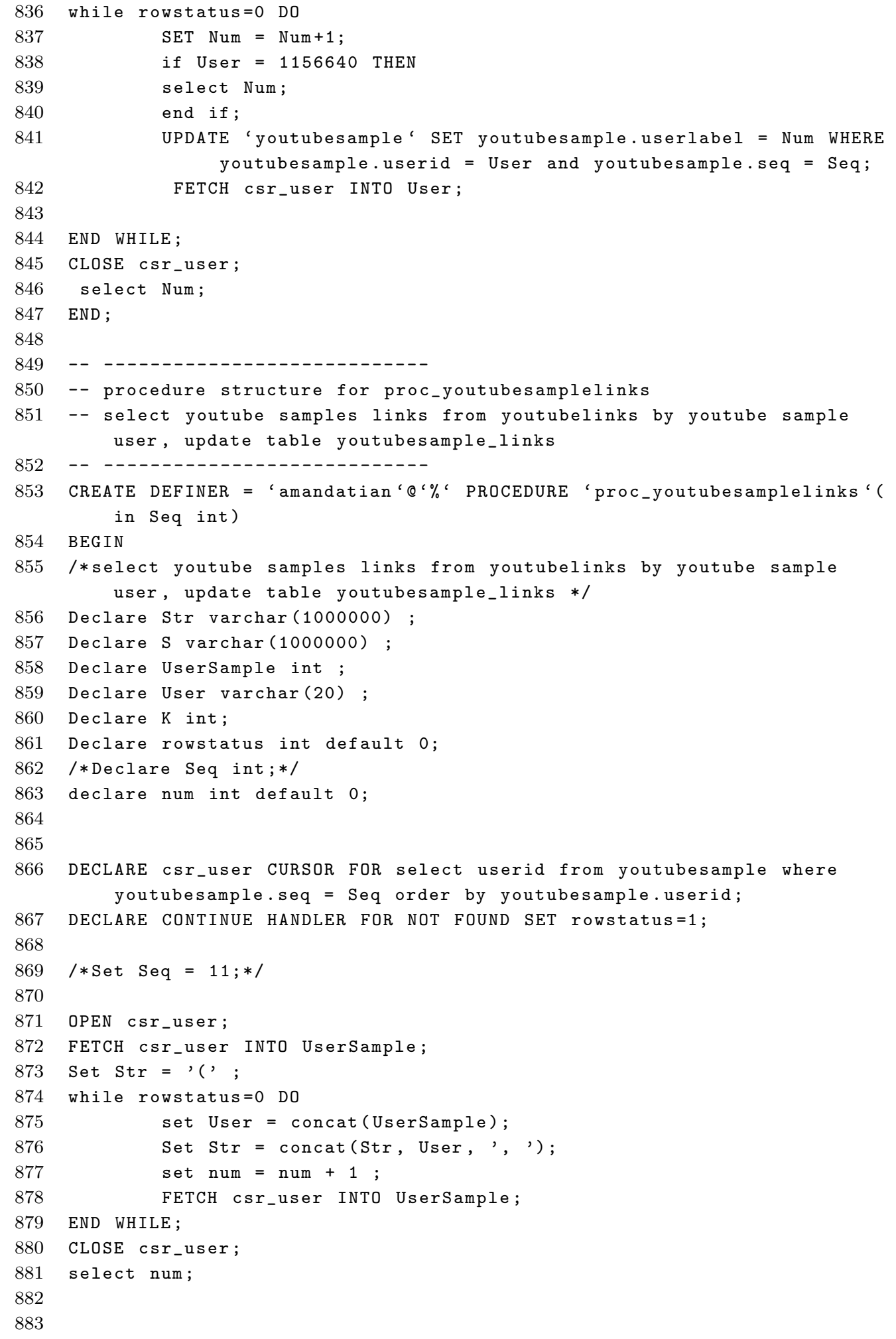




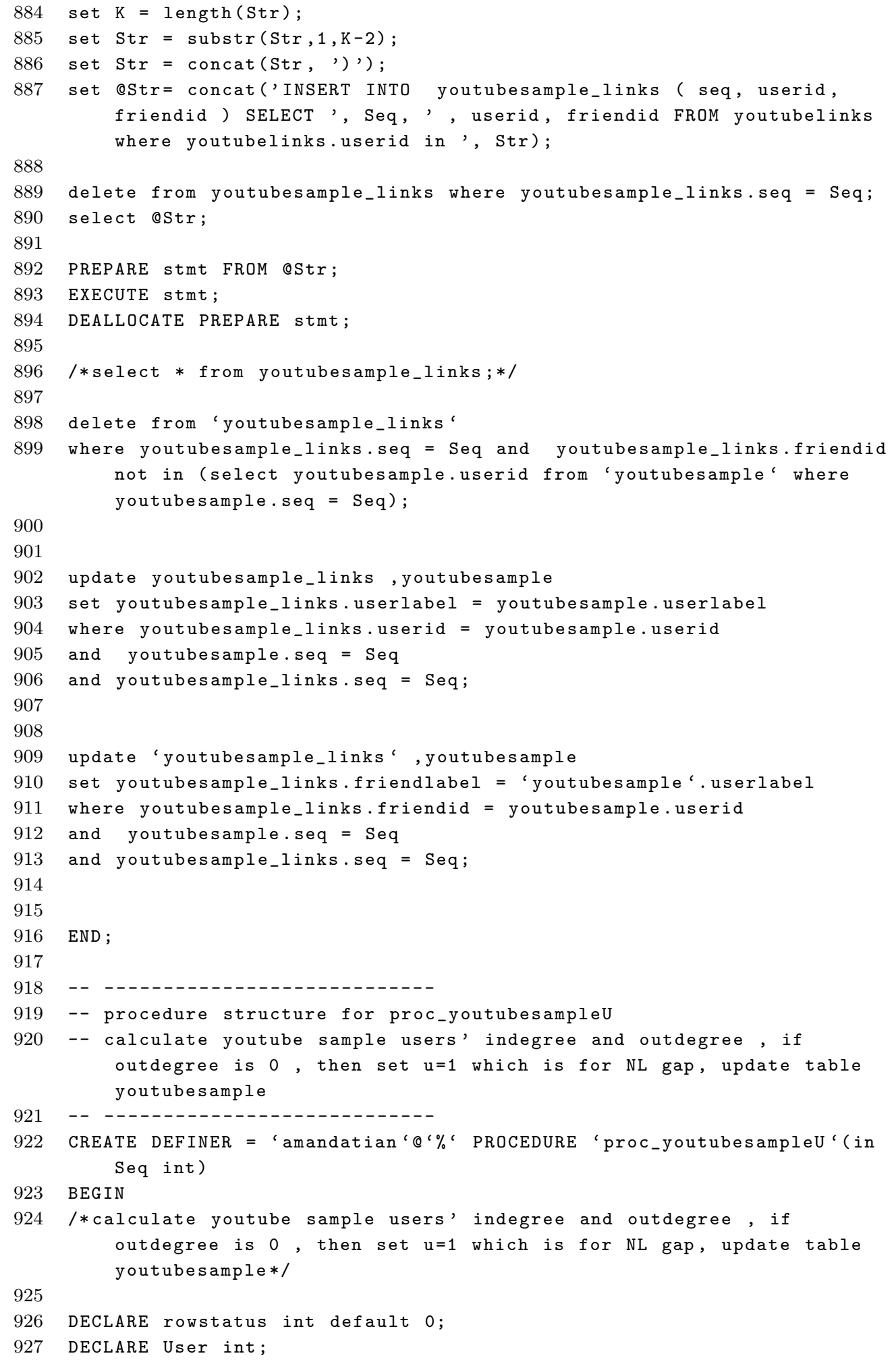




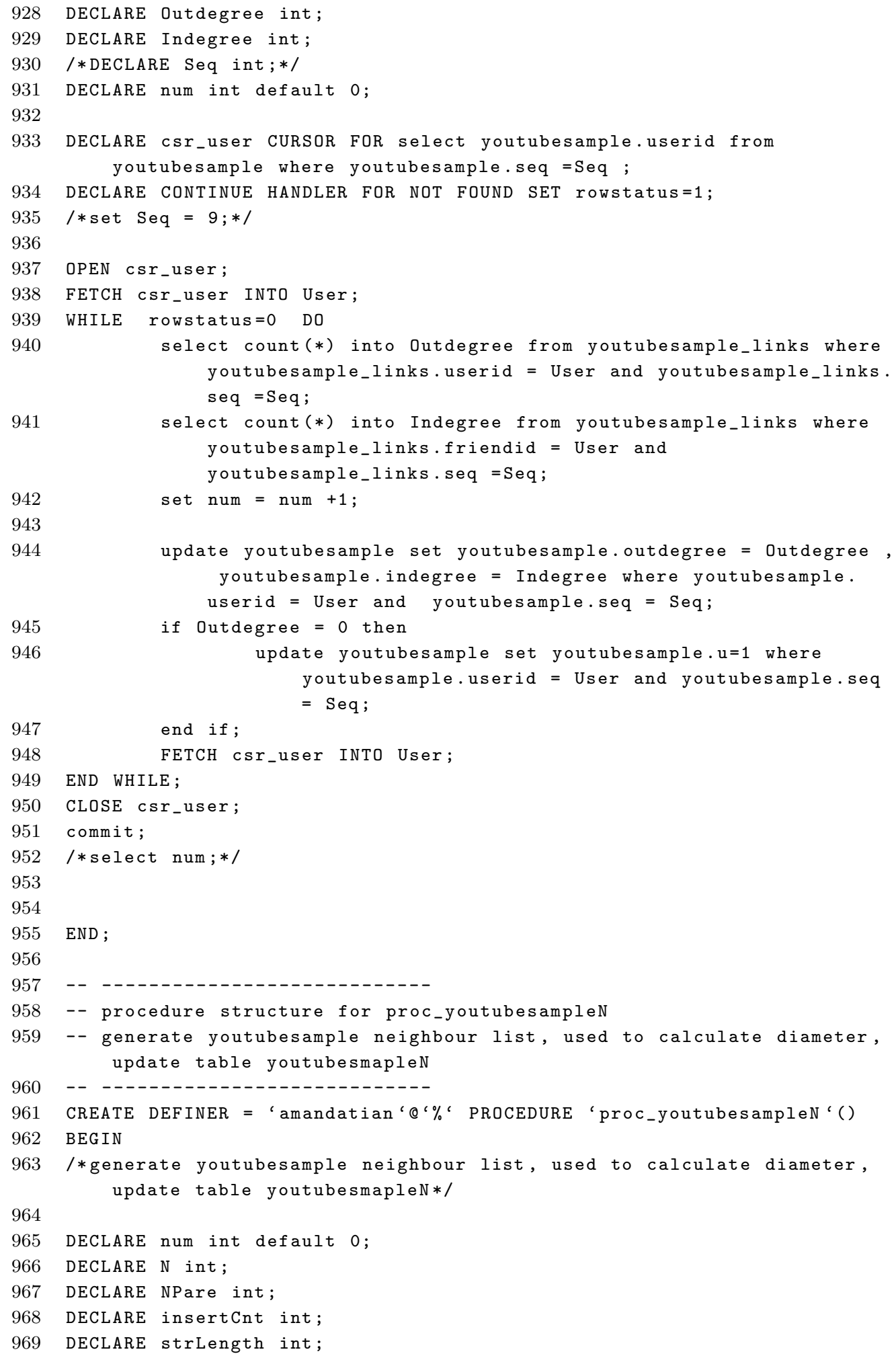

END ; select count $(*)$ into Indegree from youtubesample_links where youtubesample_links.friendid = User and youtubesample_links.seq = Seq; 


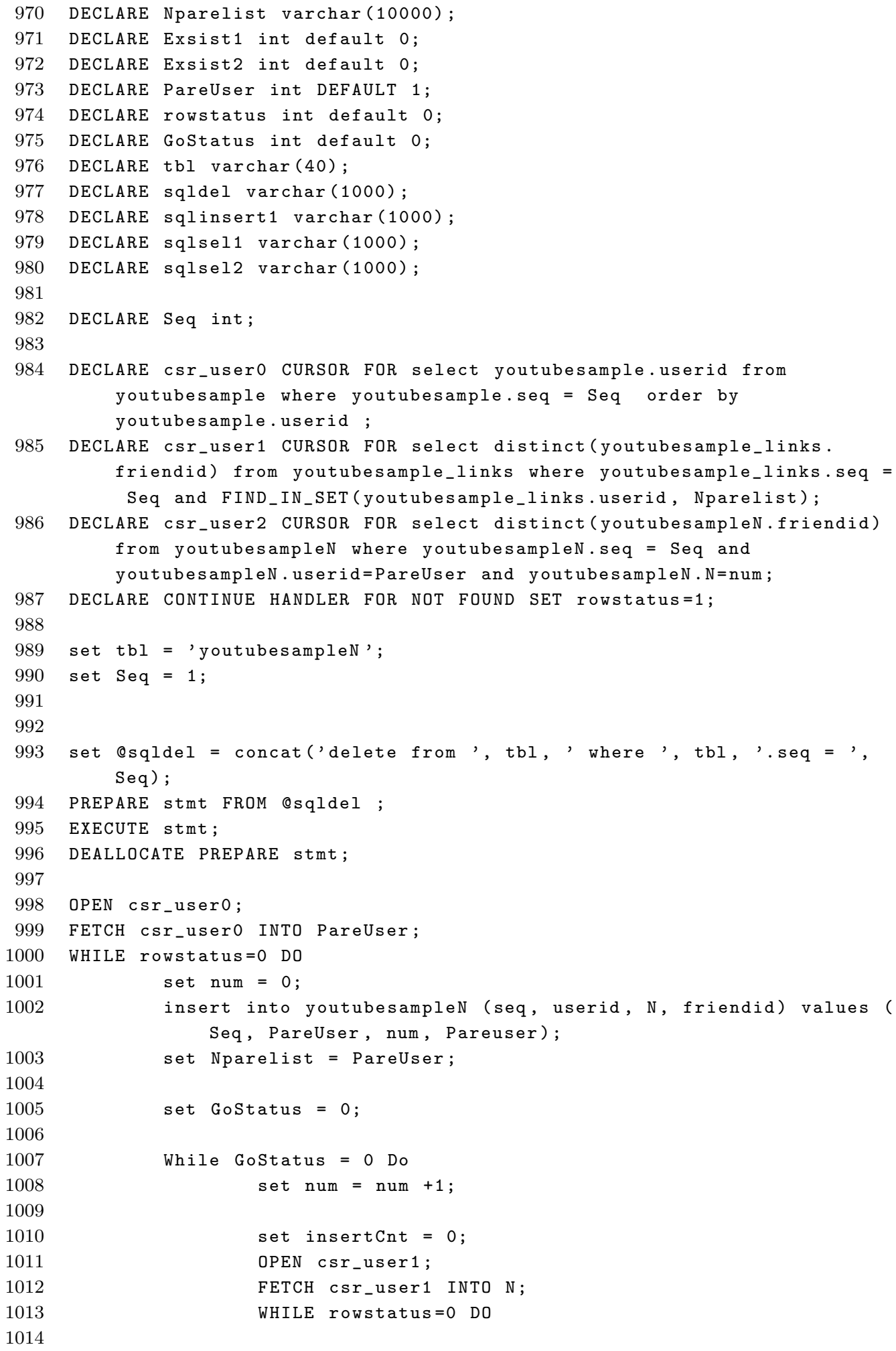




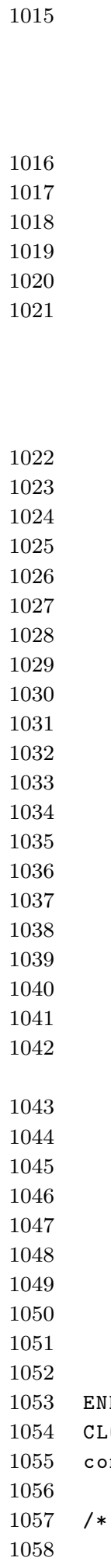




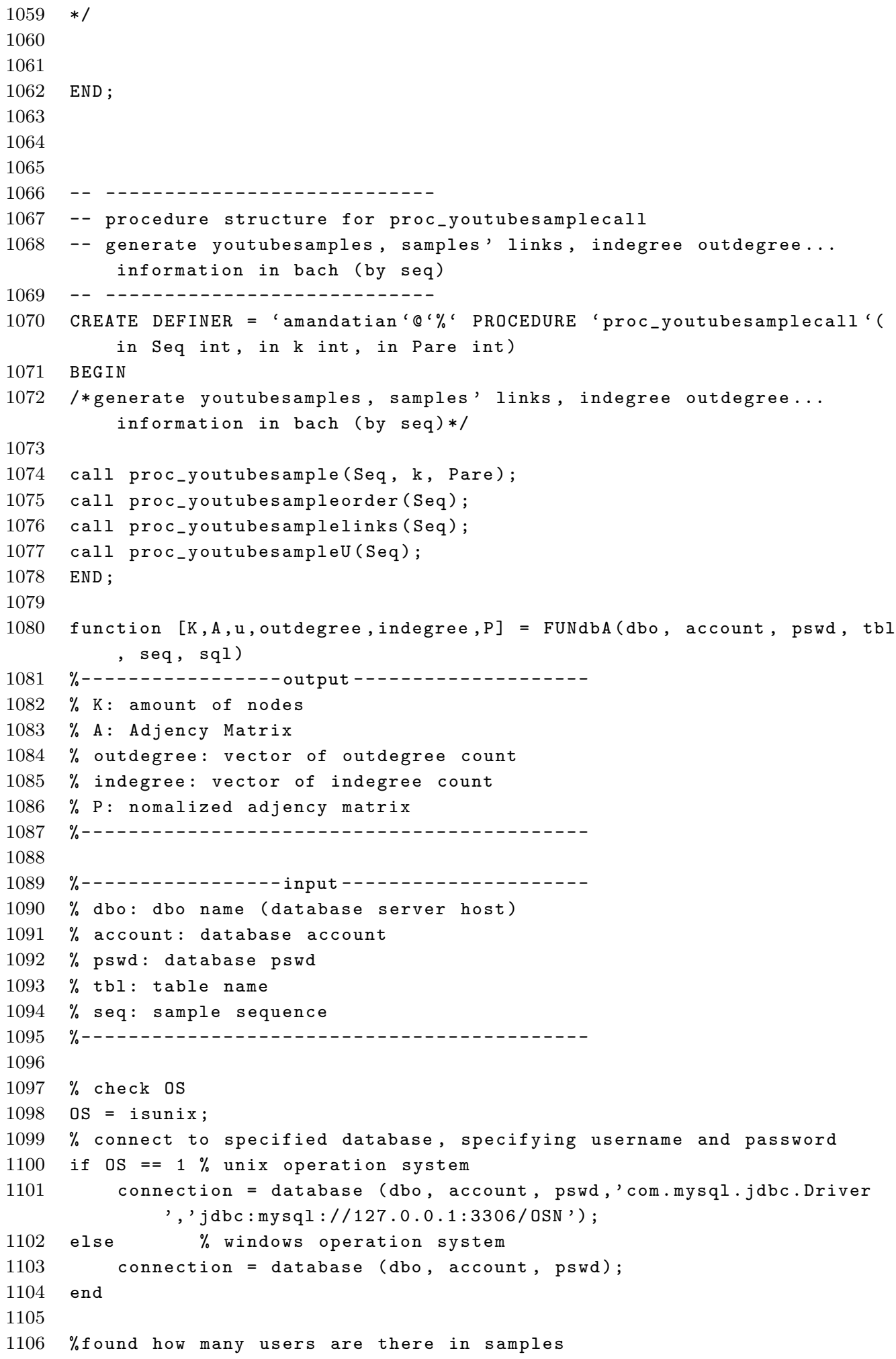




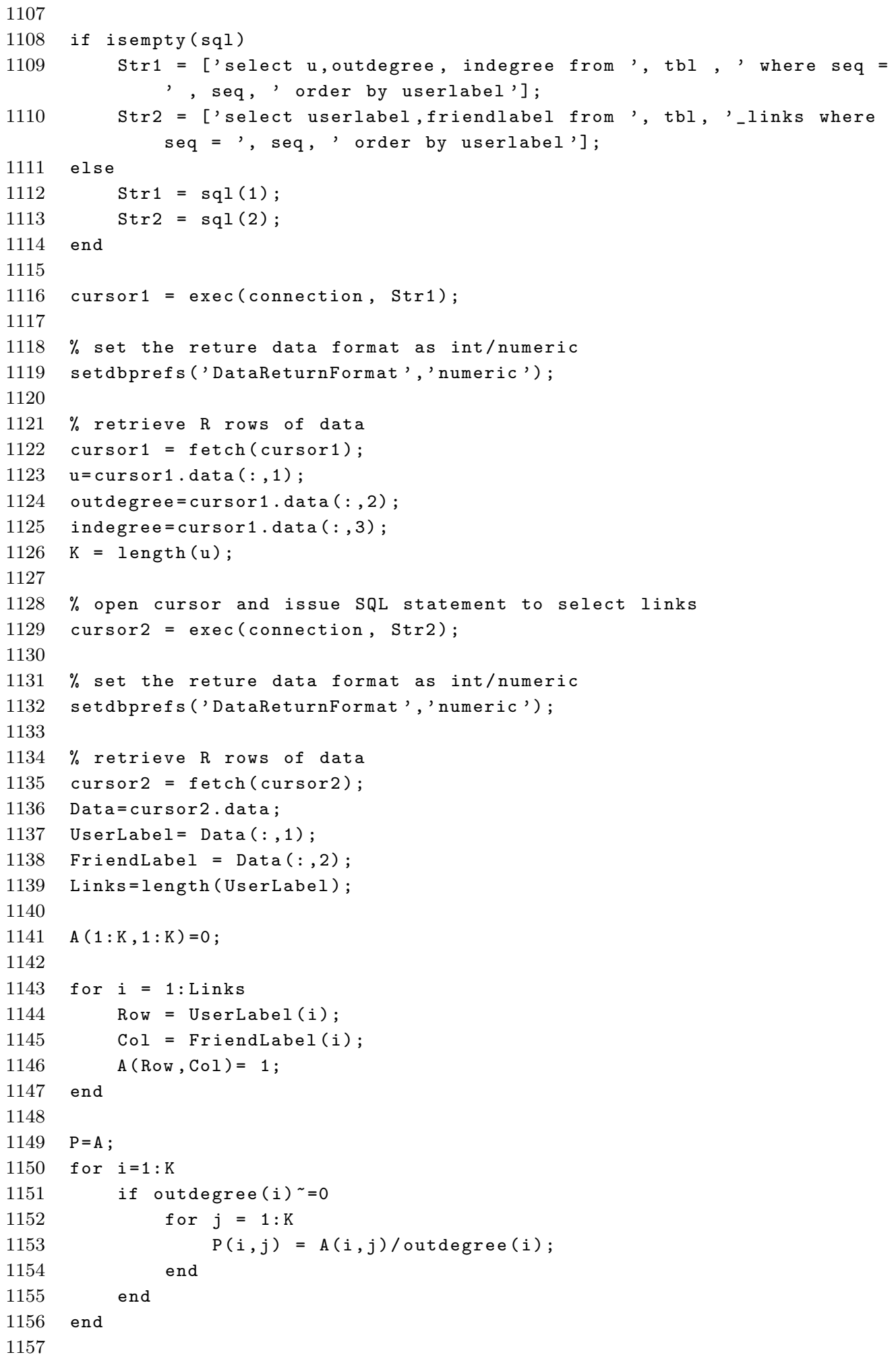

1157 


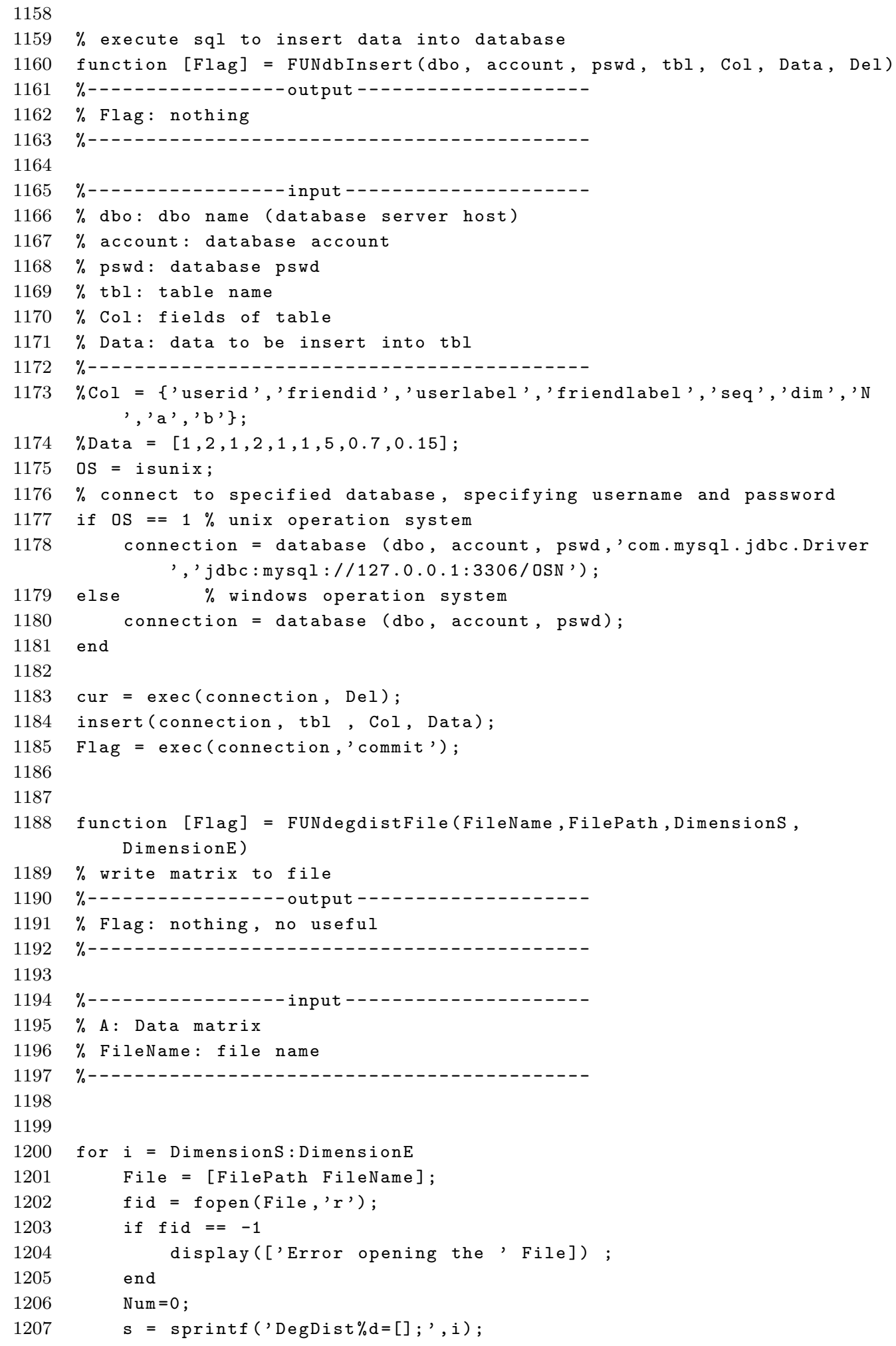




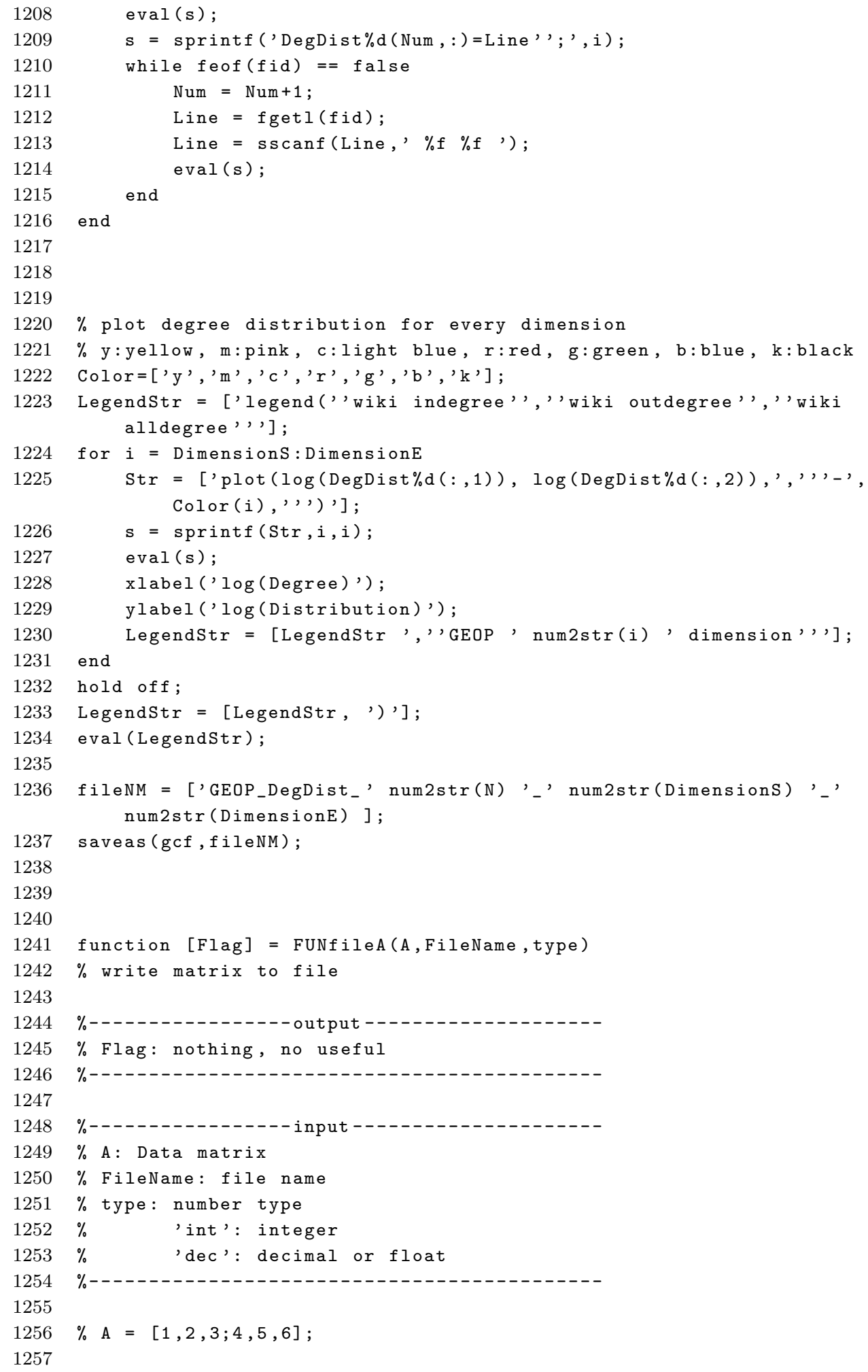




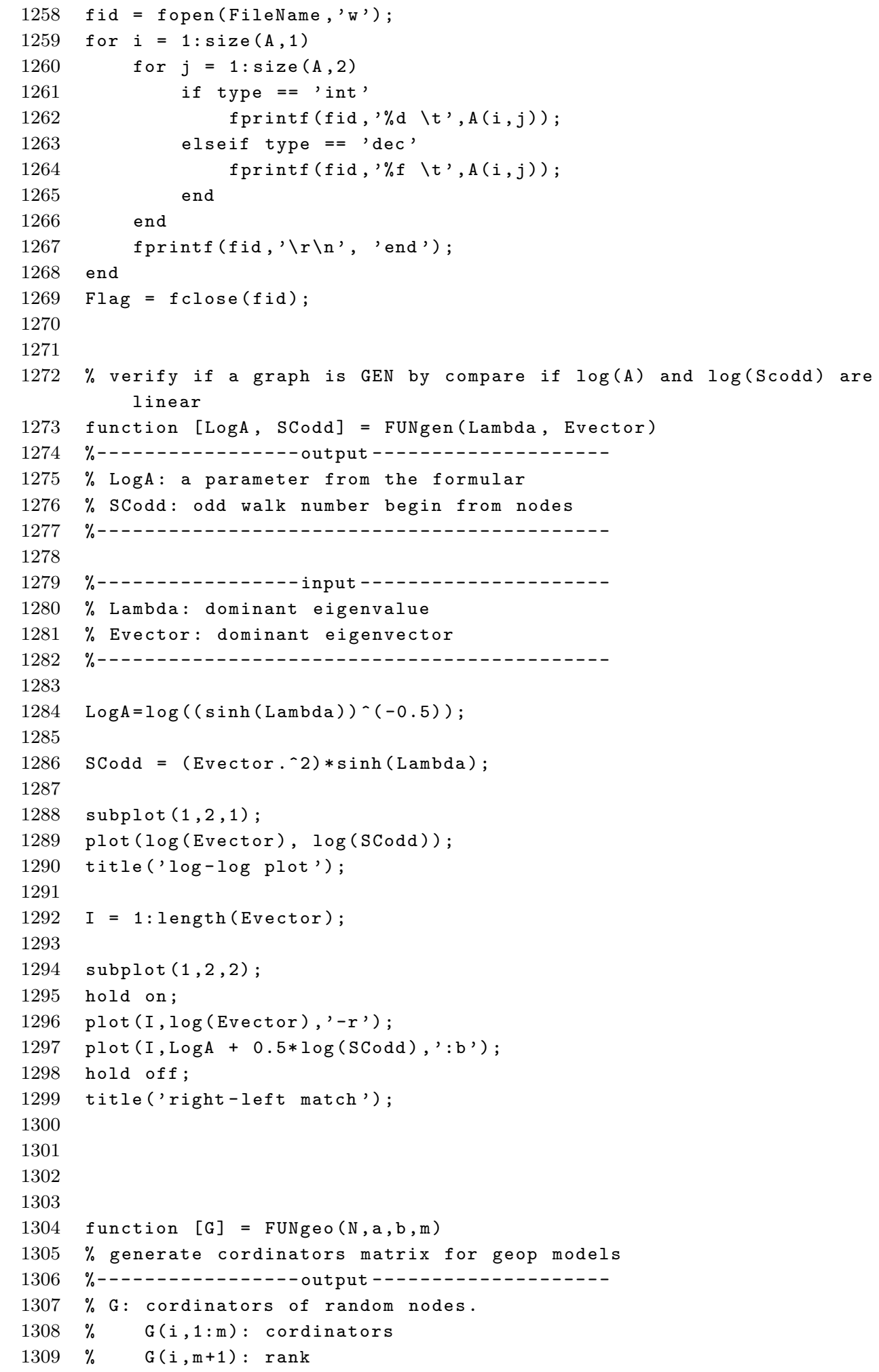

1267

1268$$
1272
$$

1277

1279

$$
1280
$$

1288

\section{9}

1301

1302

1303

1304 function [G] = Fungeo $(\mathrm{N}, \mathrm{a}, \mathrm{b}, \mathrm{m})$

$1305 \%$ generate cordinators matrix for geop models

1306 \%----------------output-------------------

$1307 \%$ G: cordinators of random nodes.

$1308 \%$ G(i, $1: m)$ : cordinators

$1309 \% \quad G(i, m+1):$ rank 


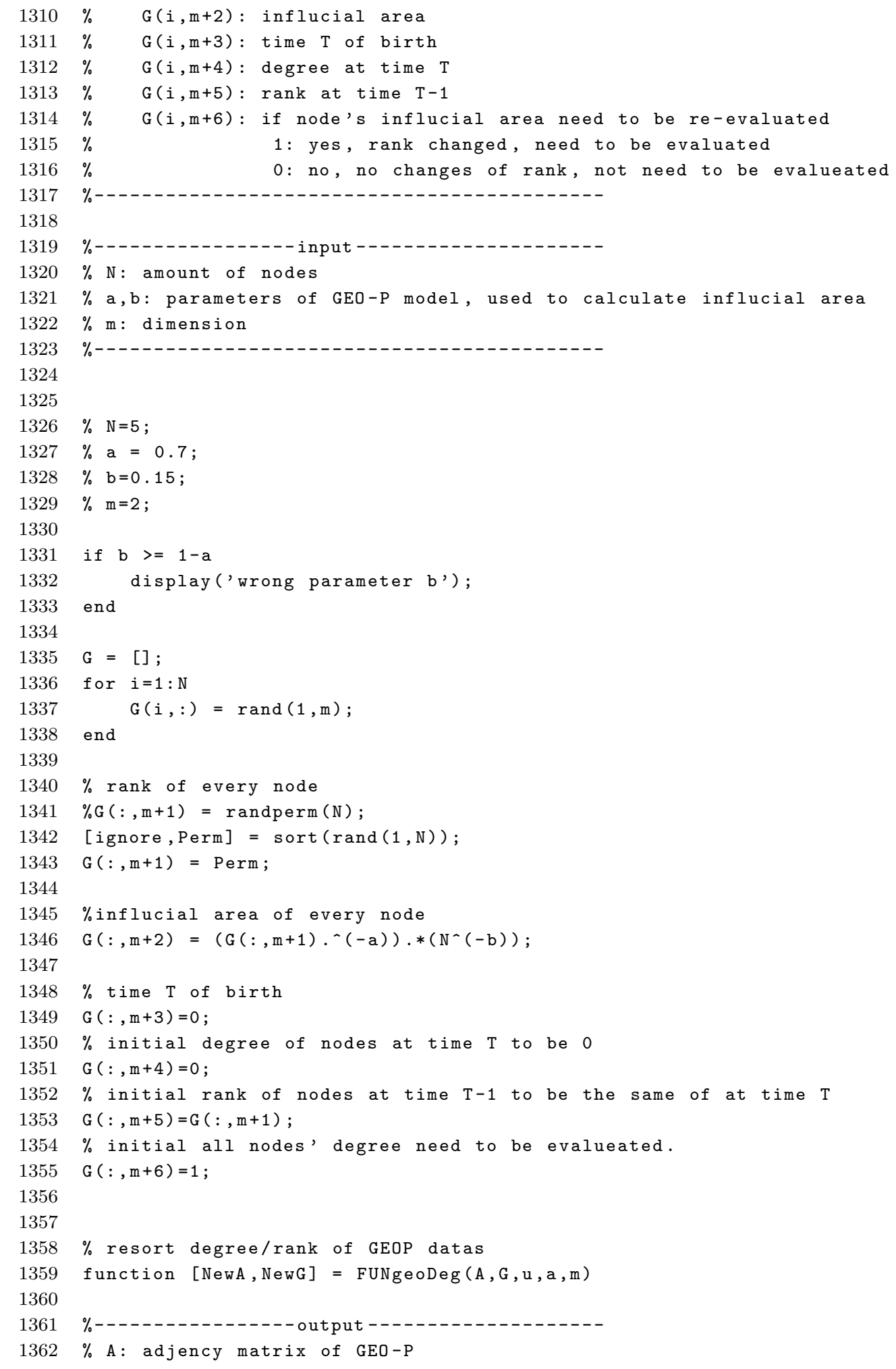




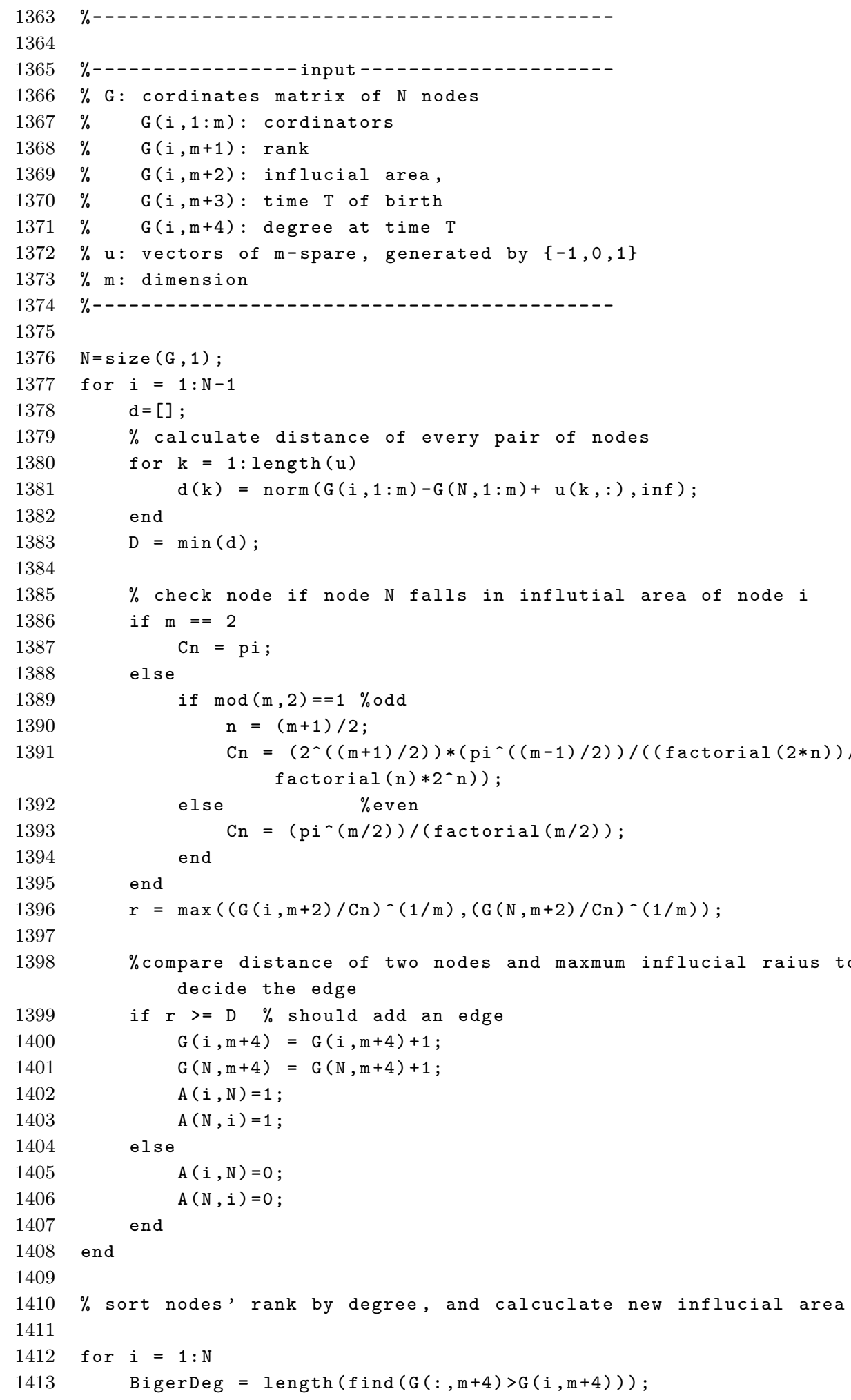




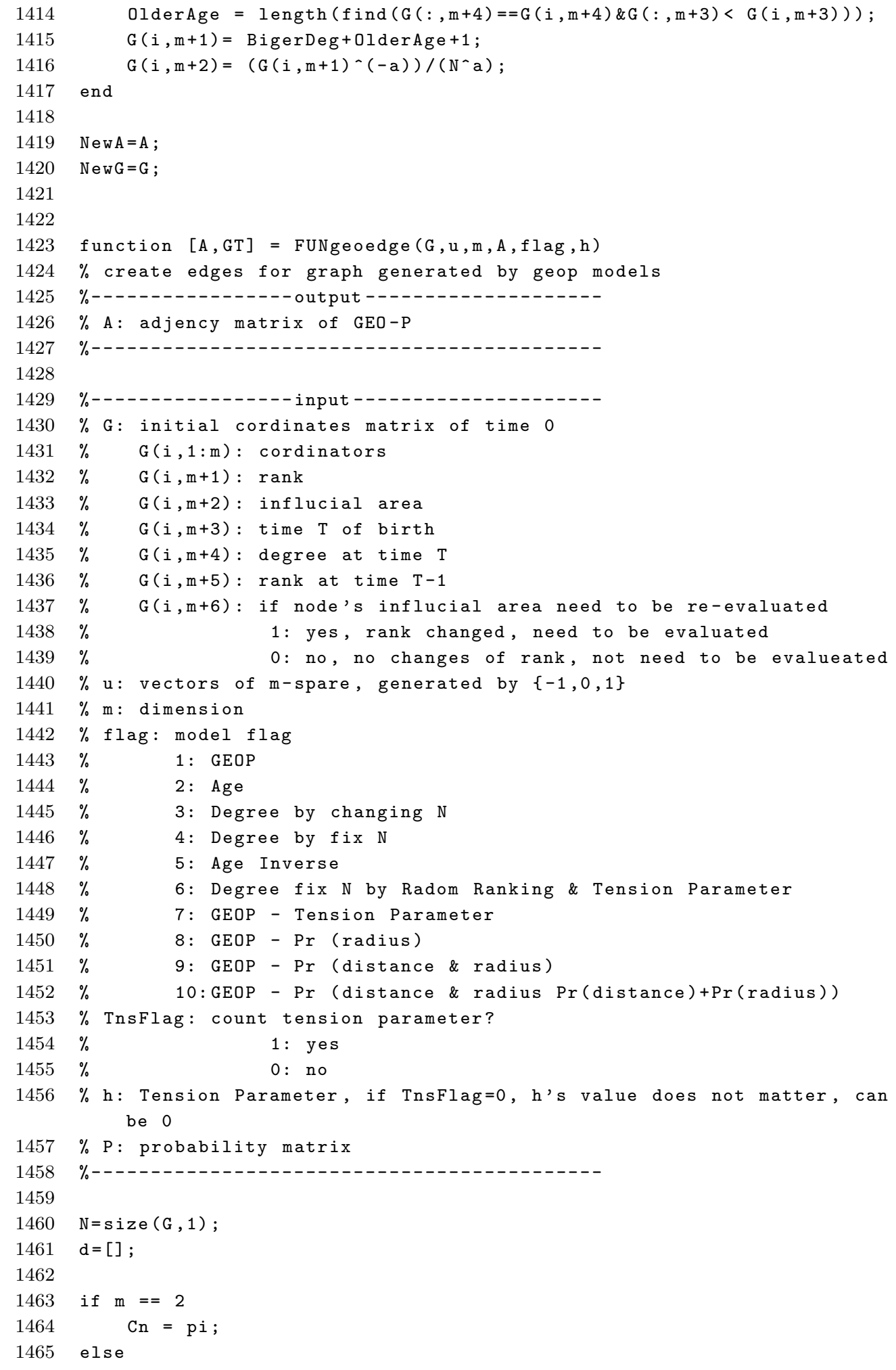




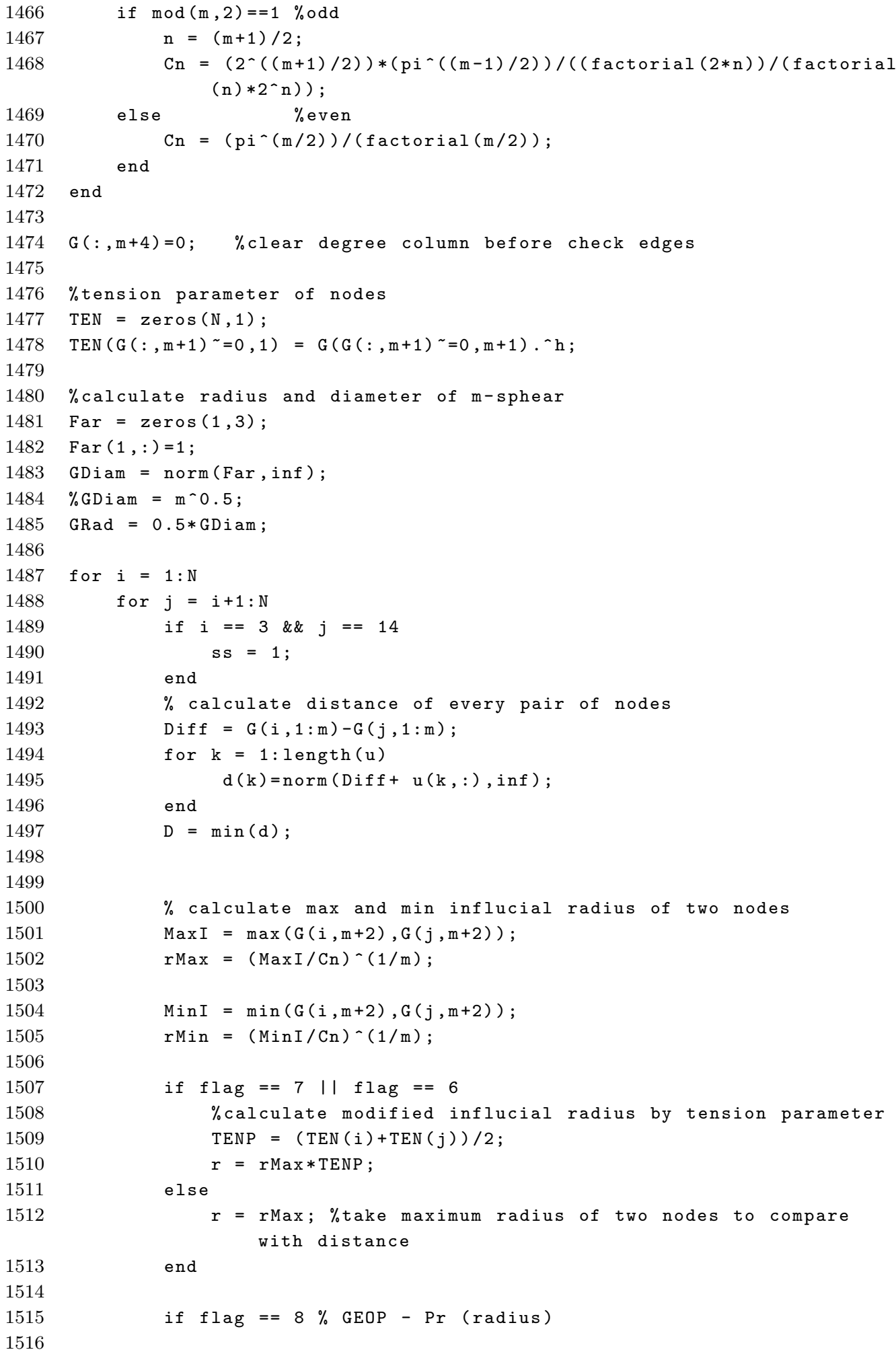




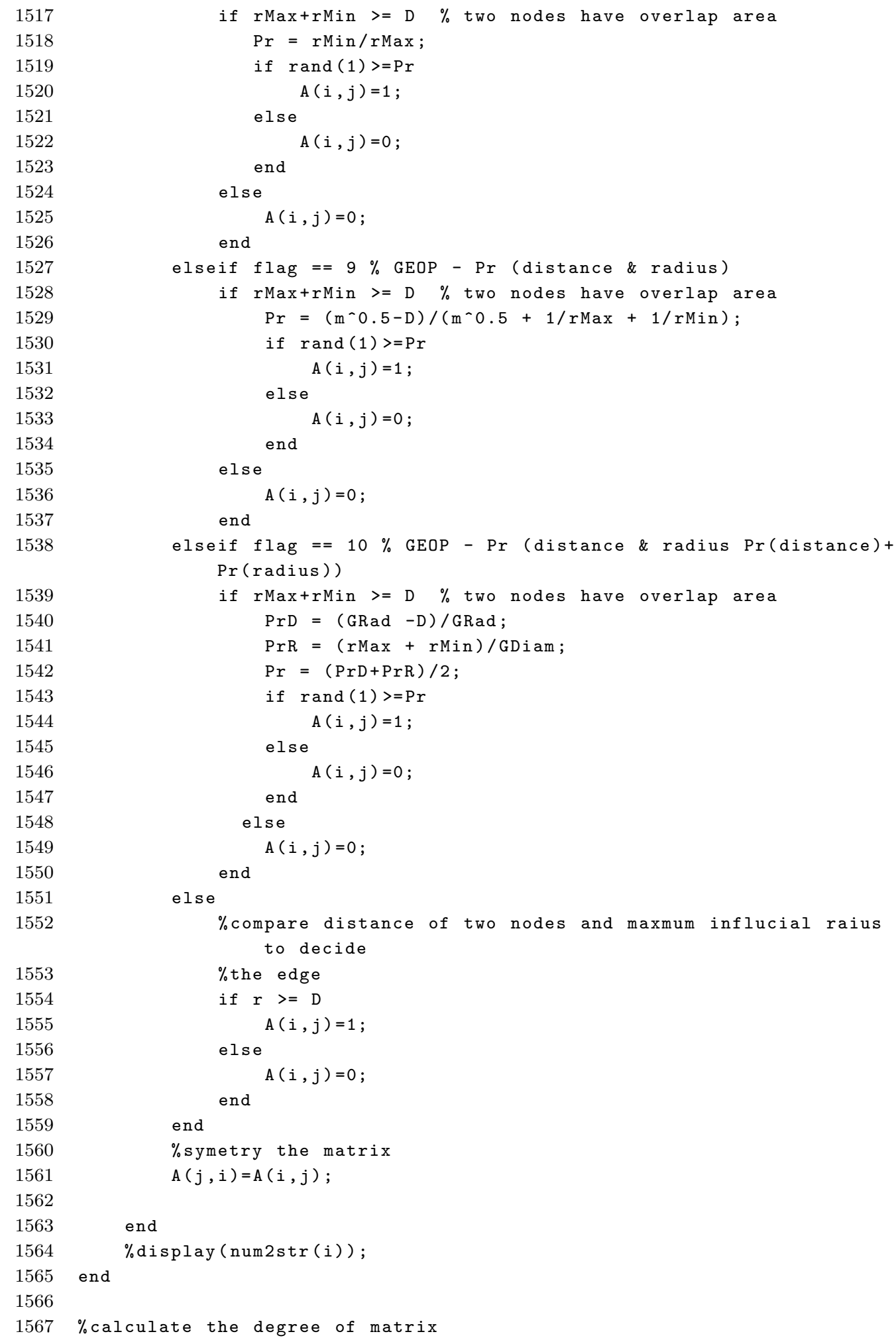




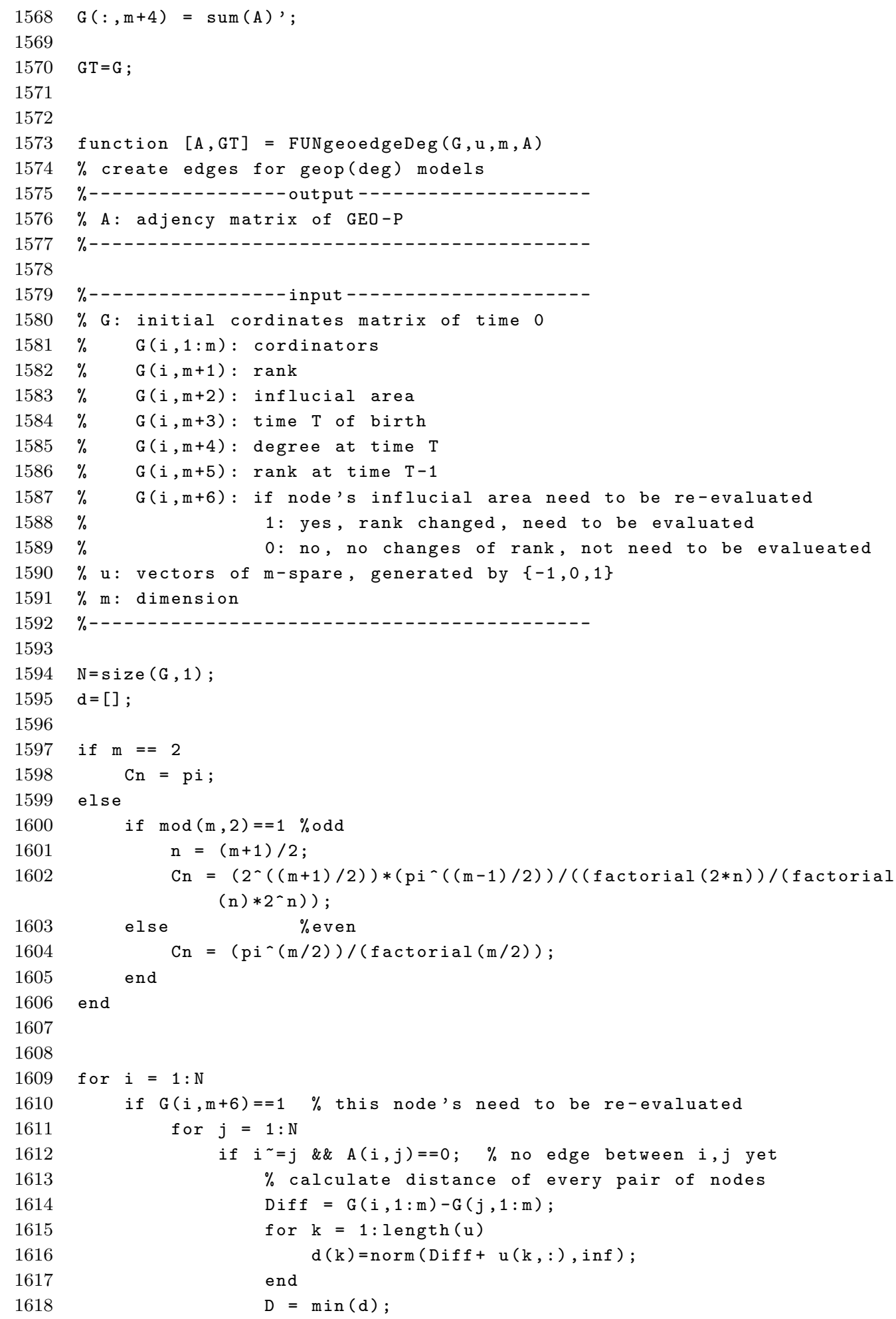




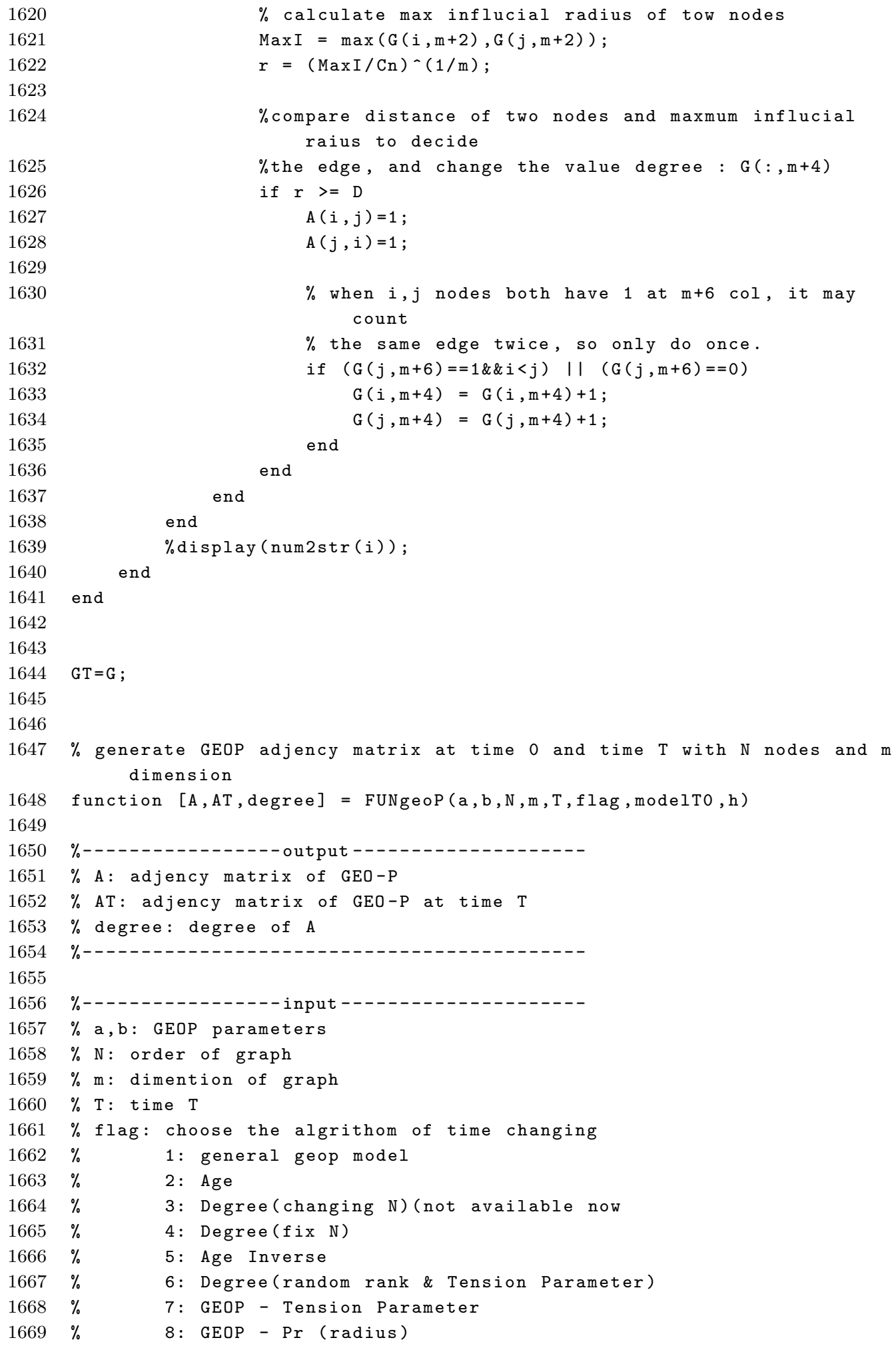




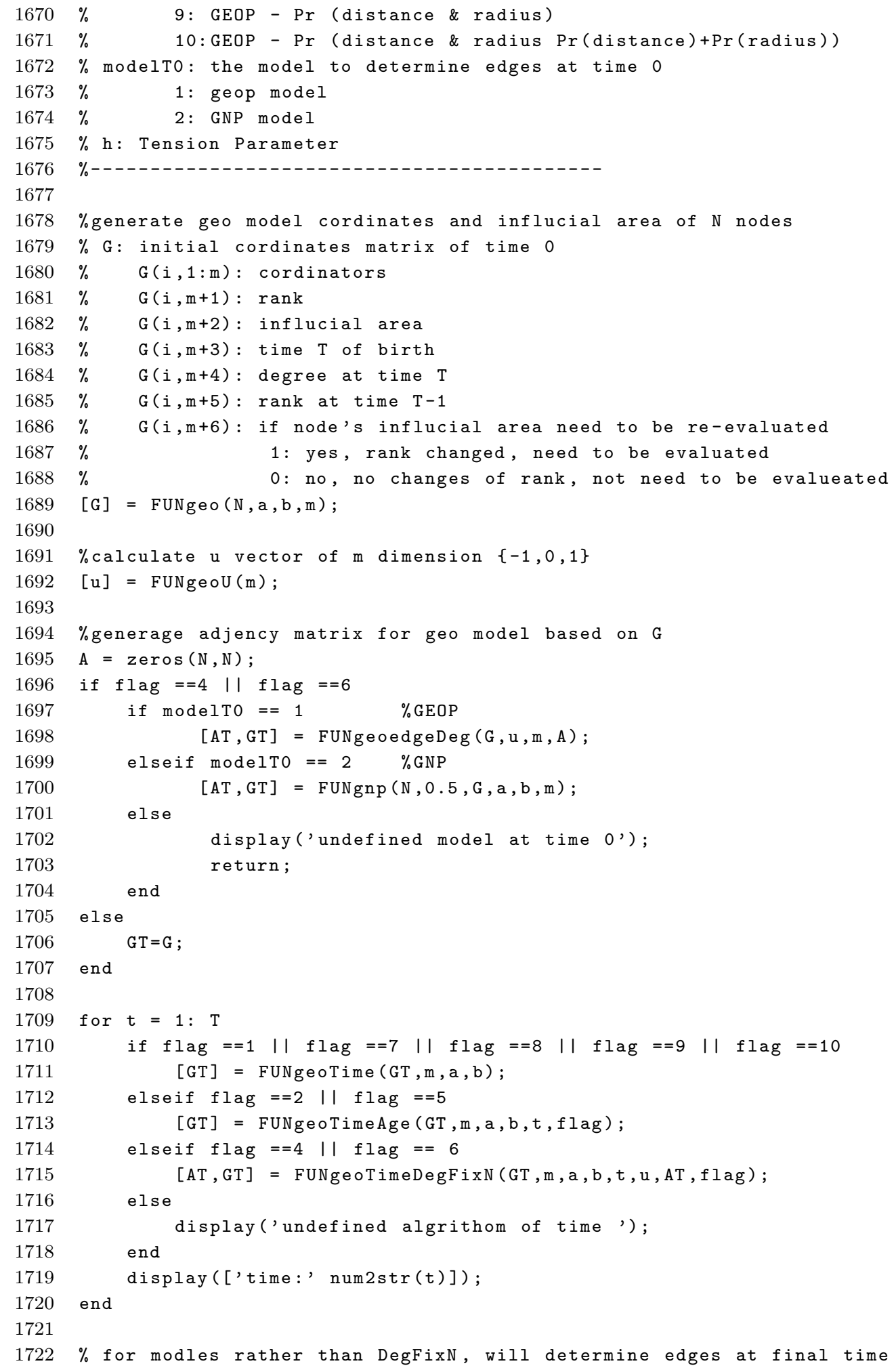




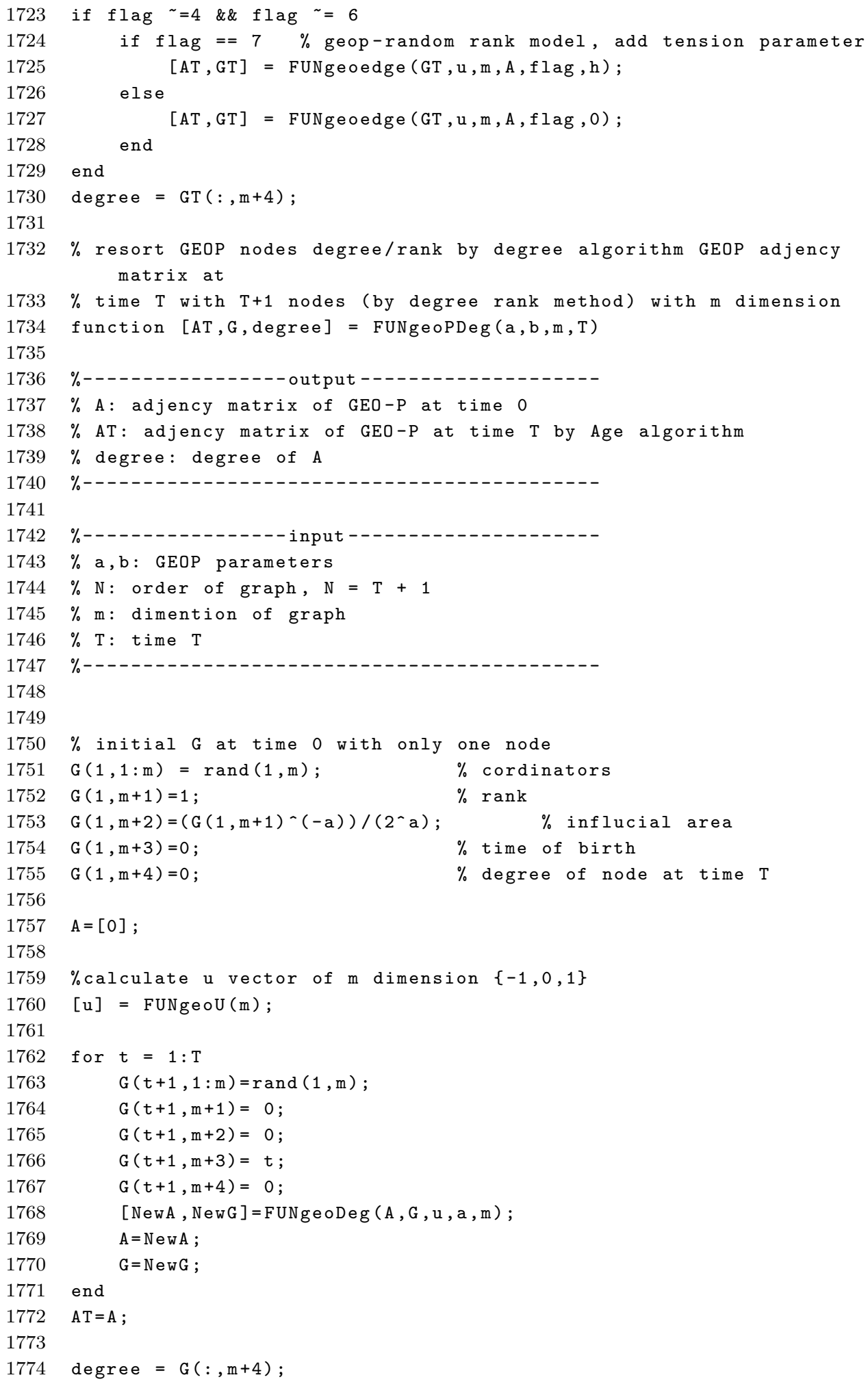

1740

1741

1742

1748

1749

1750

1751

1752

1753

1754

1755

1756

1757

1758

1759

1760

1761

1762

1763

1764

1765

1766

1767

1768

1769

1770

1771

1772

1773

1774 


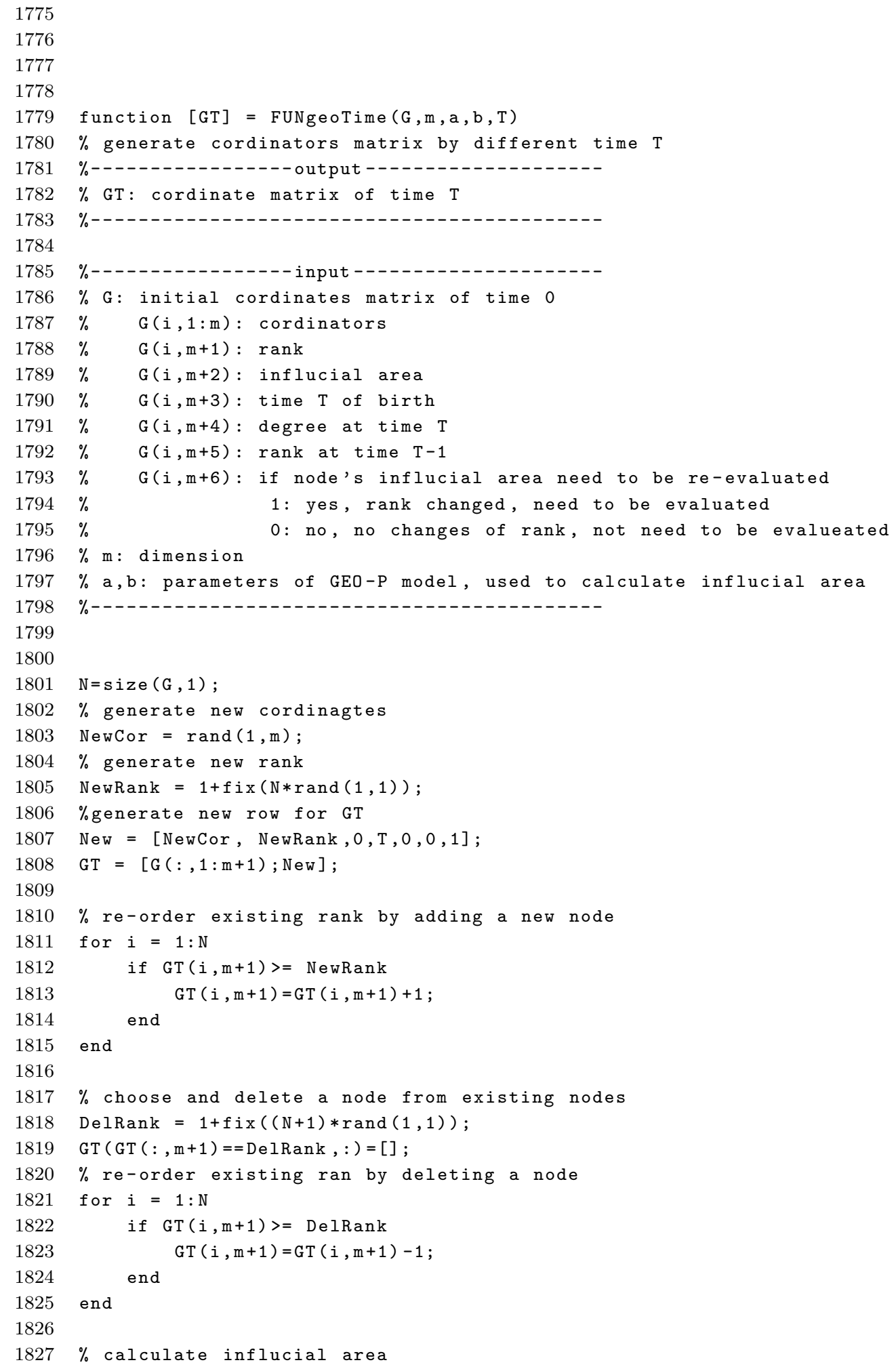




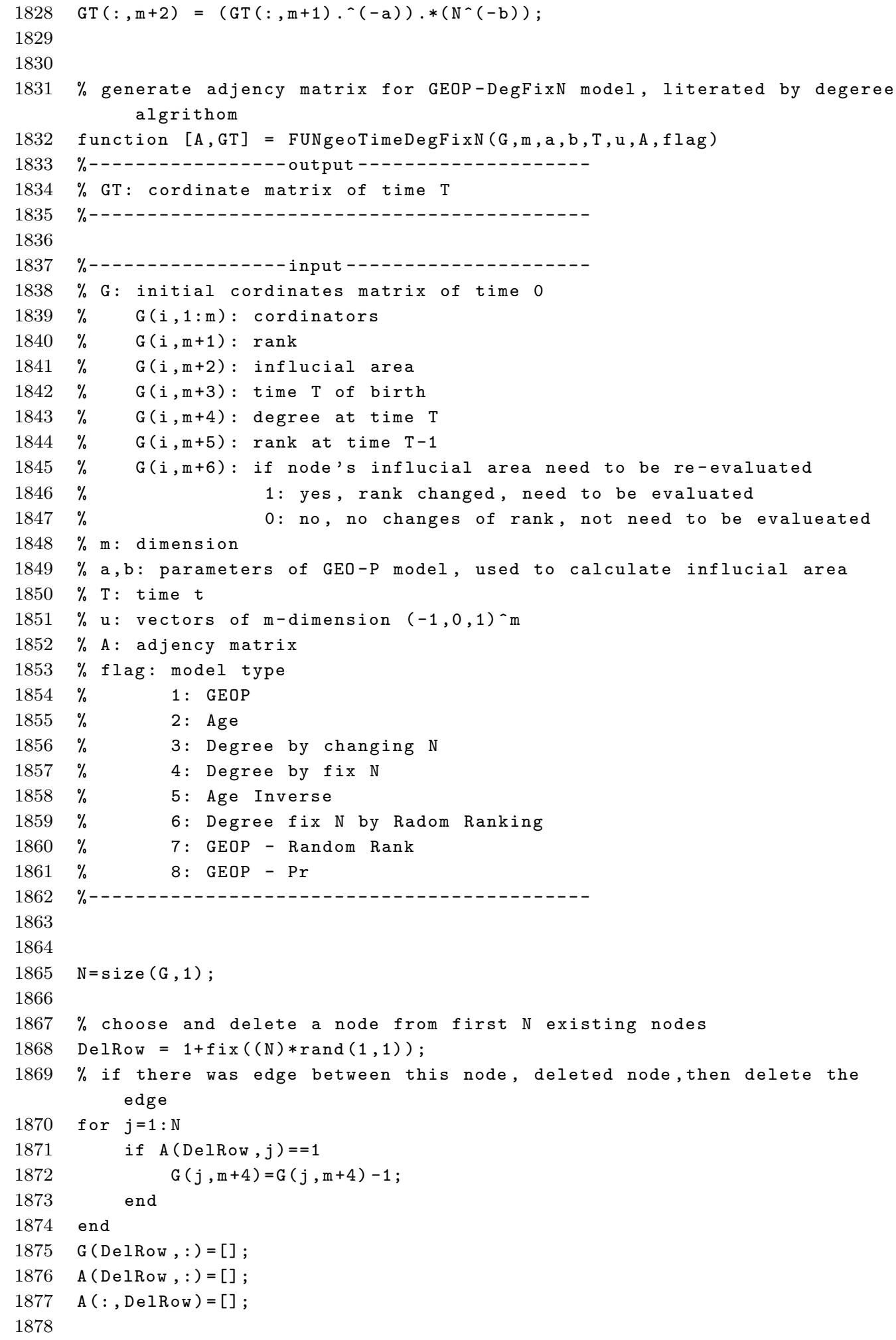




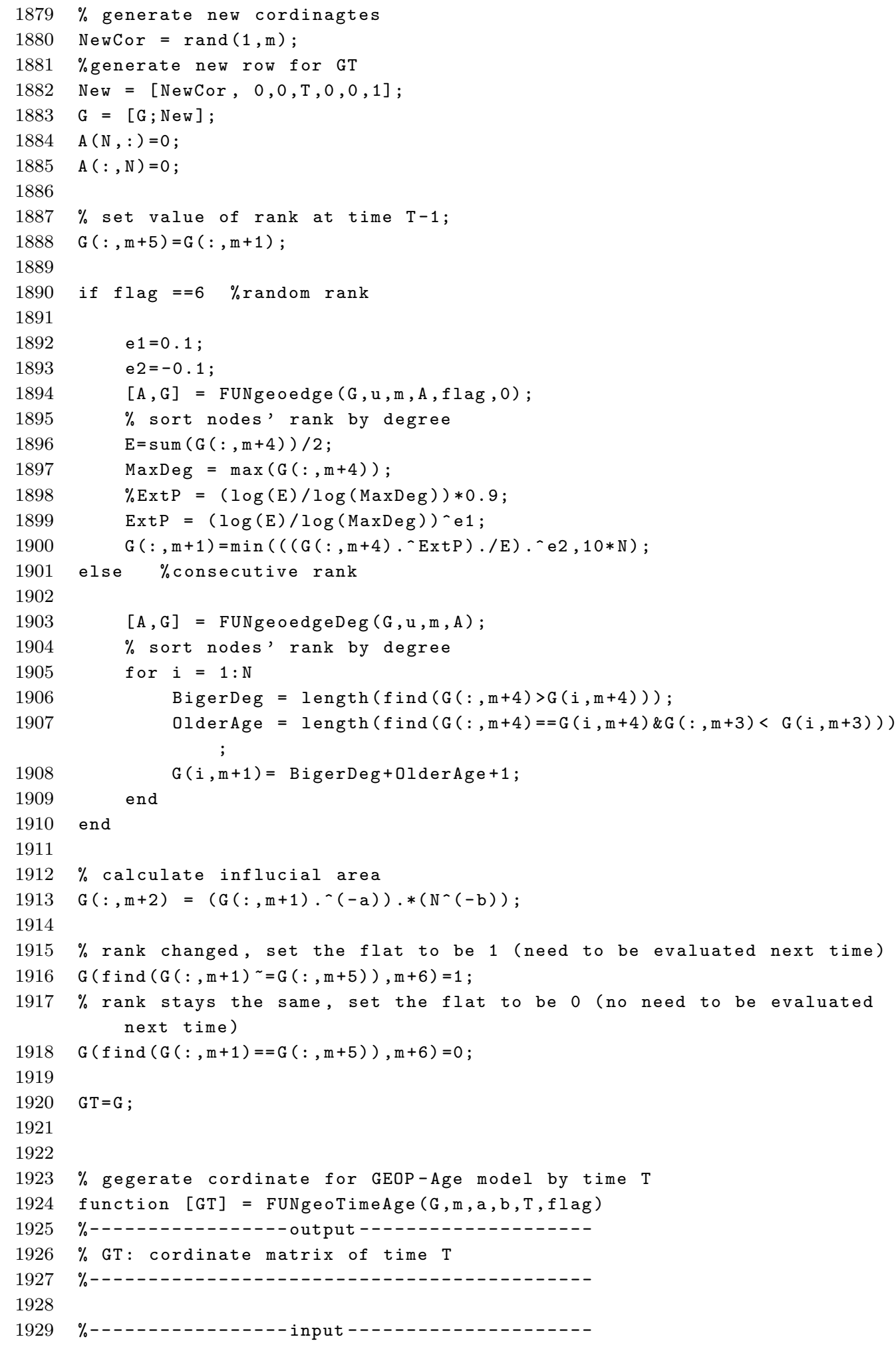




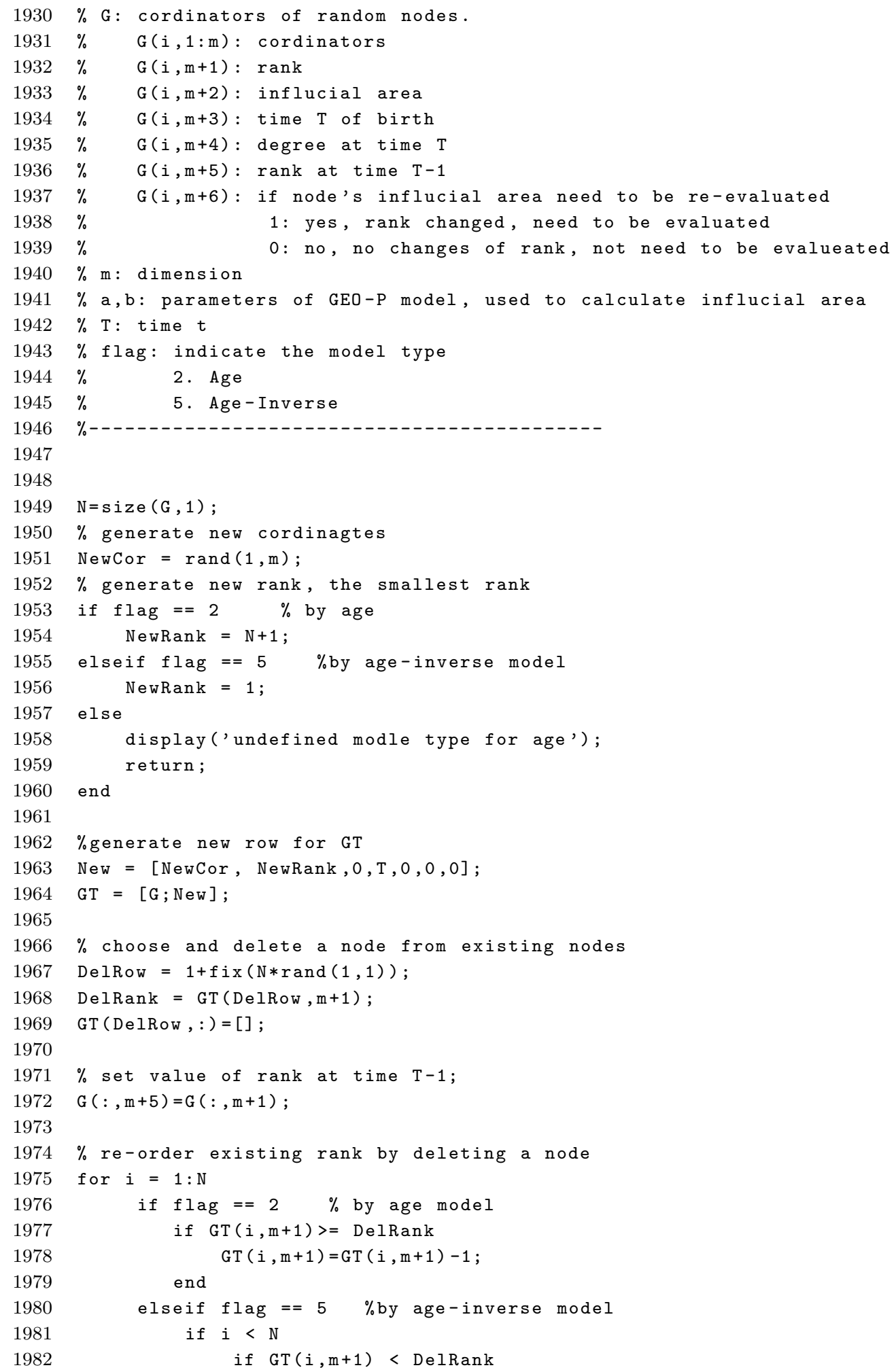




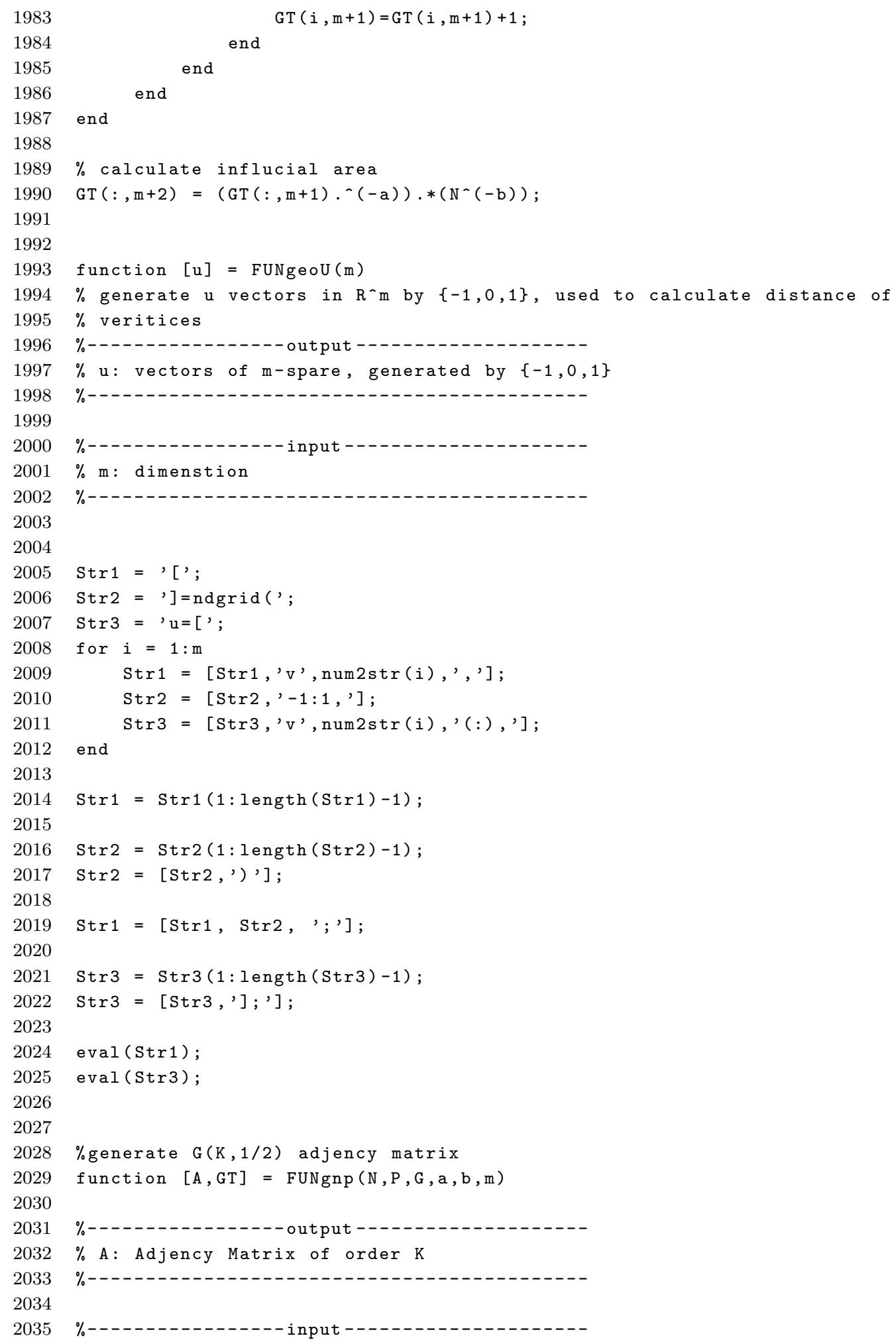




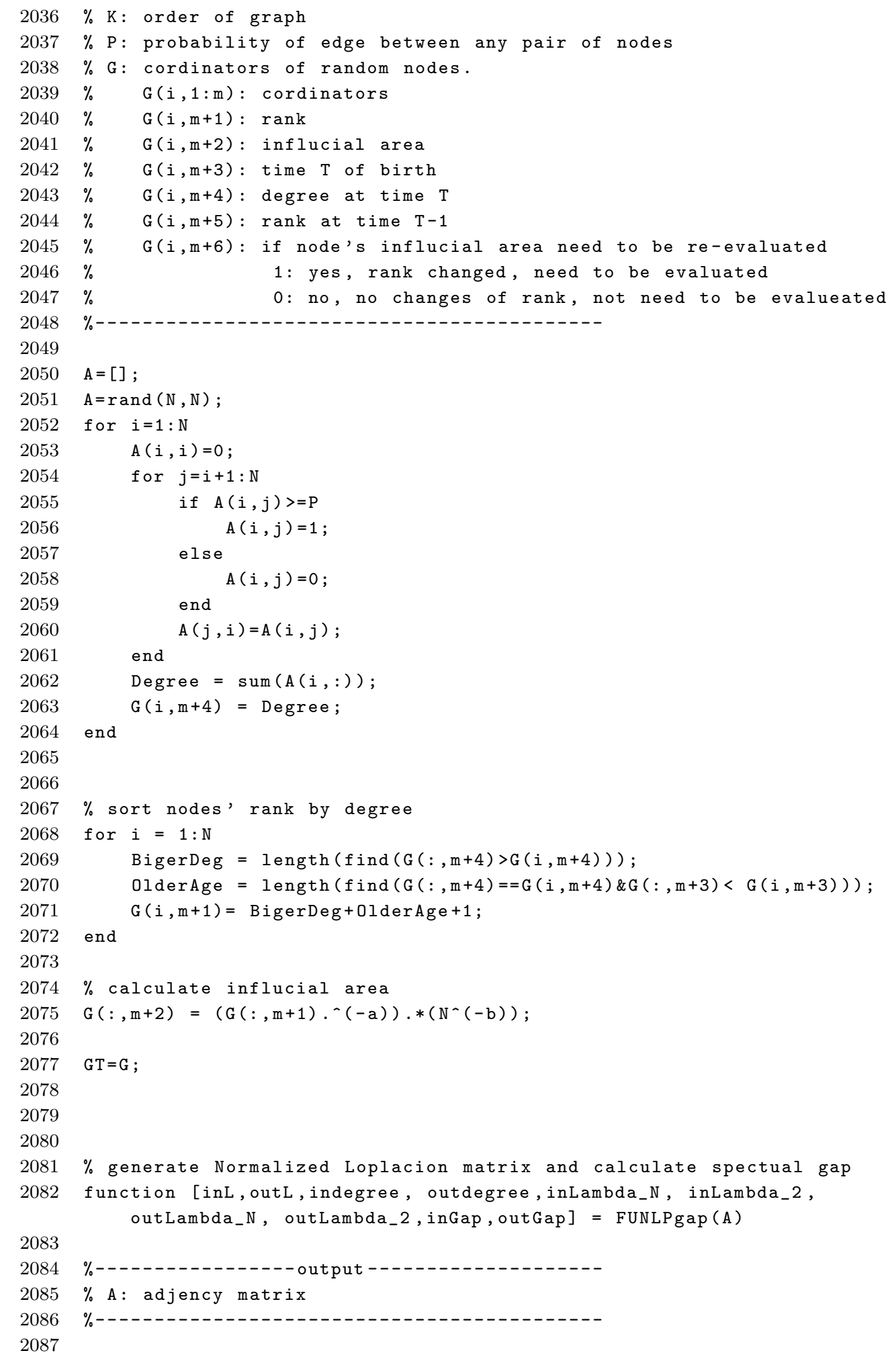

2087 


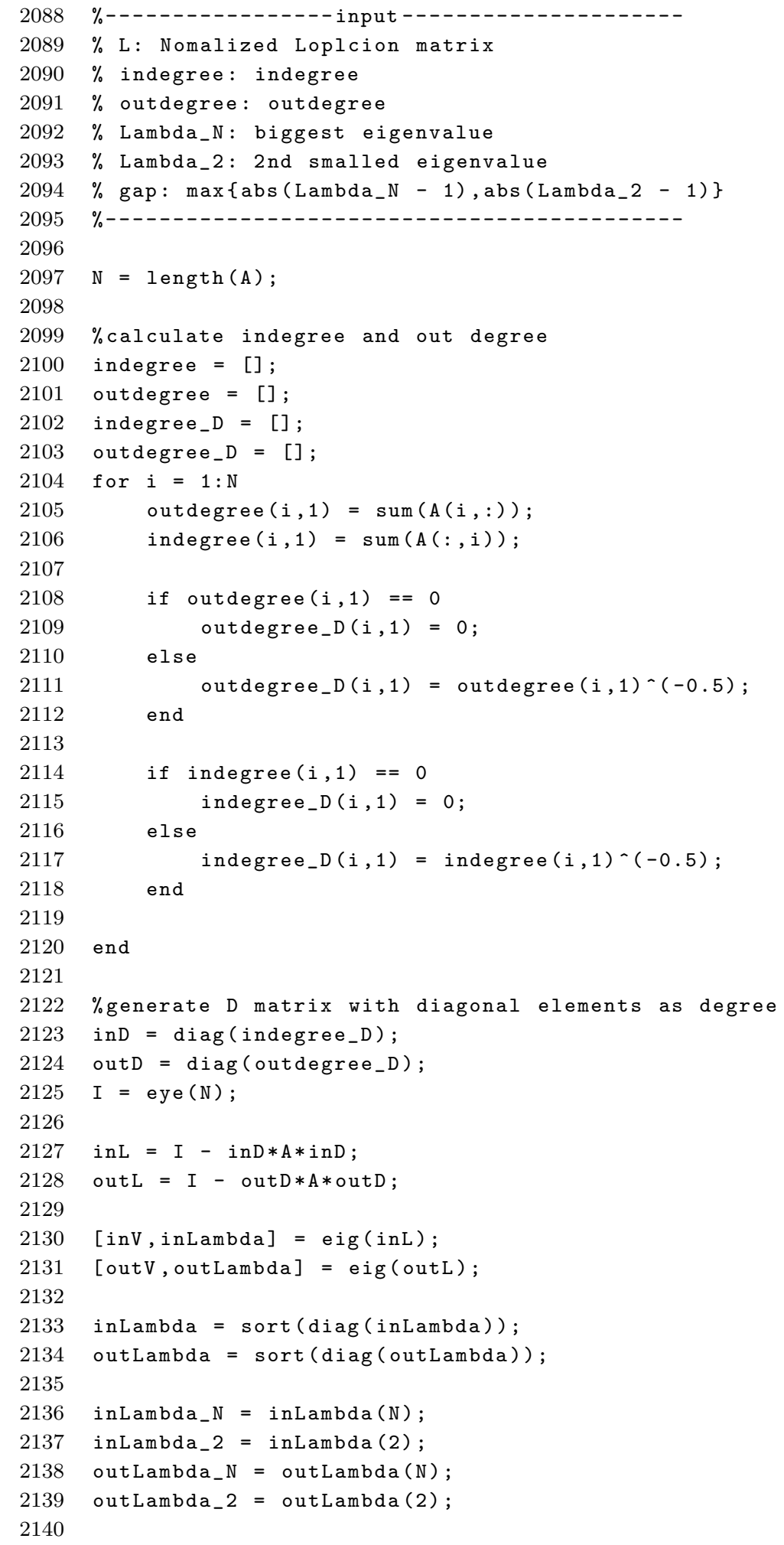




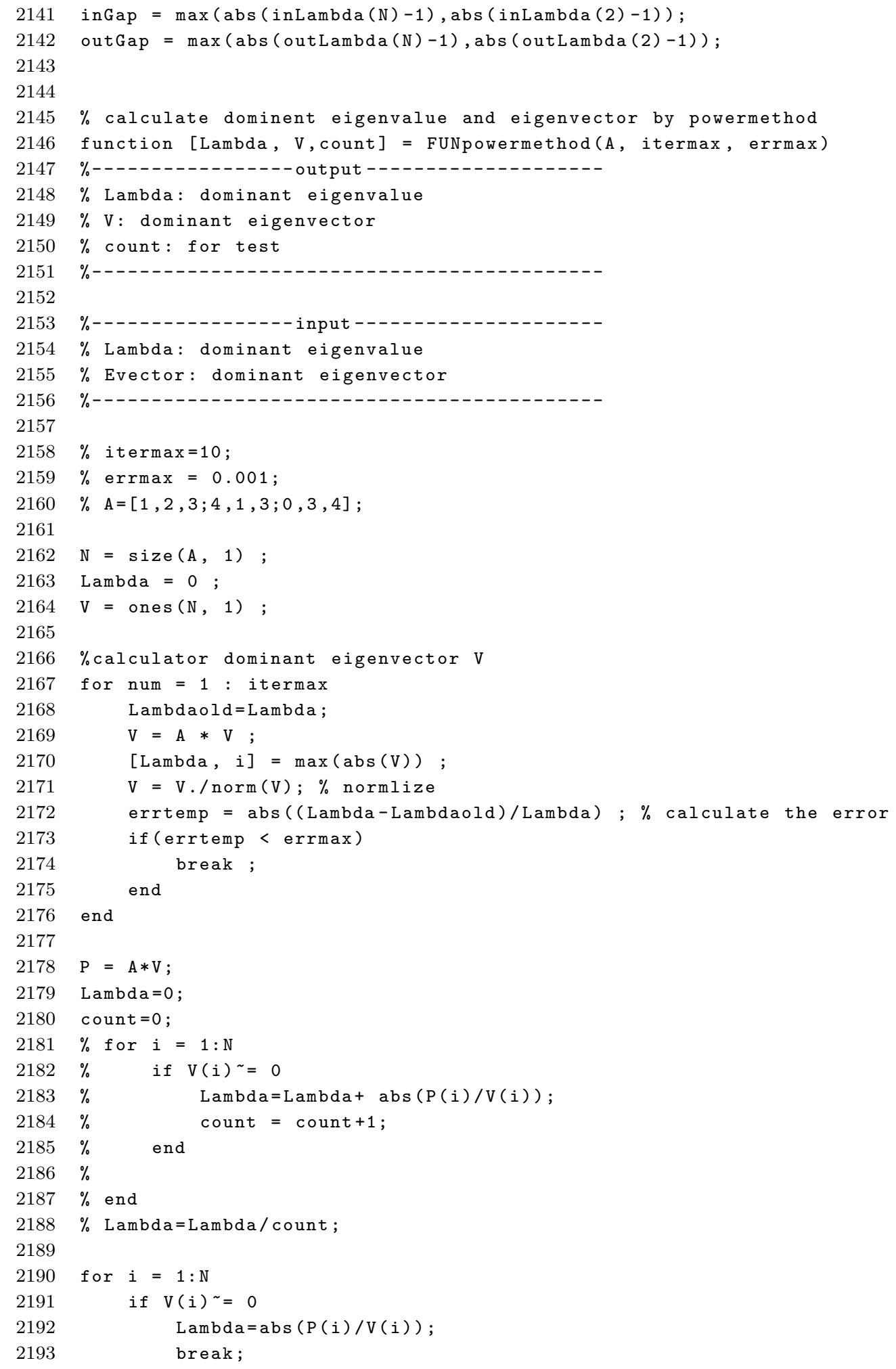




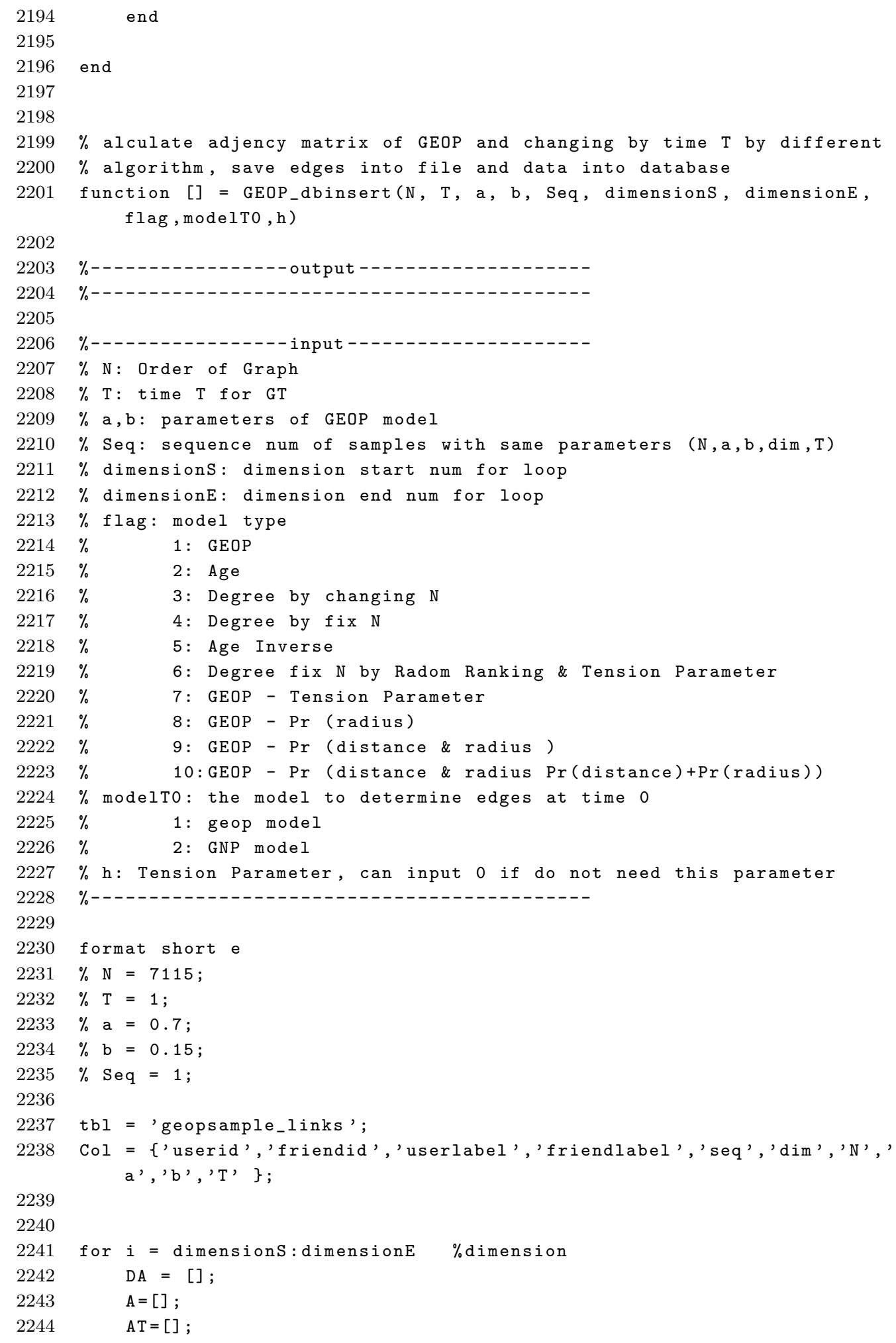




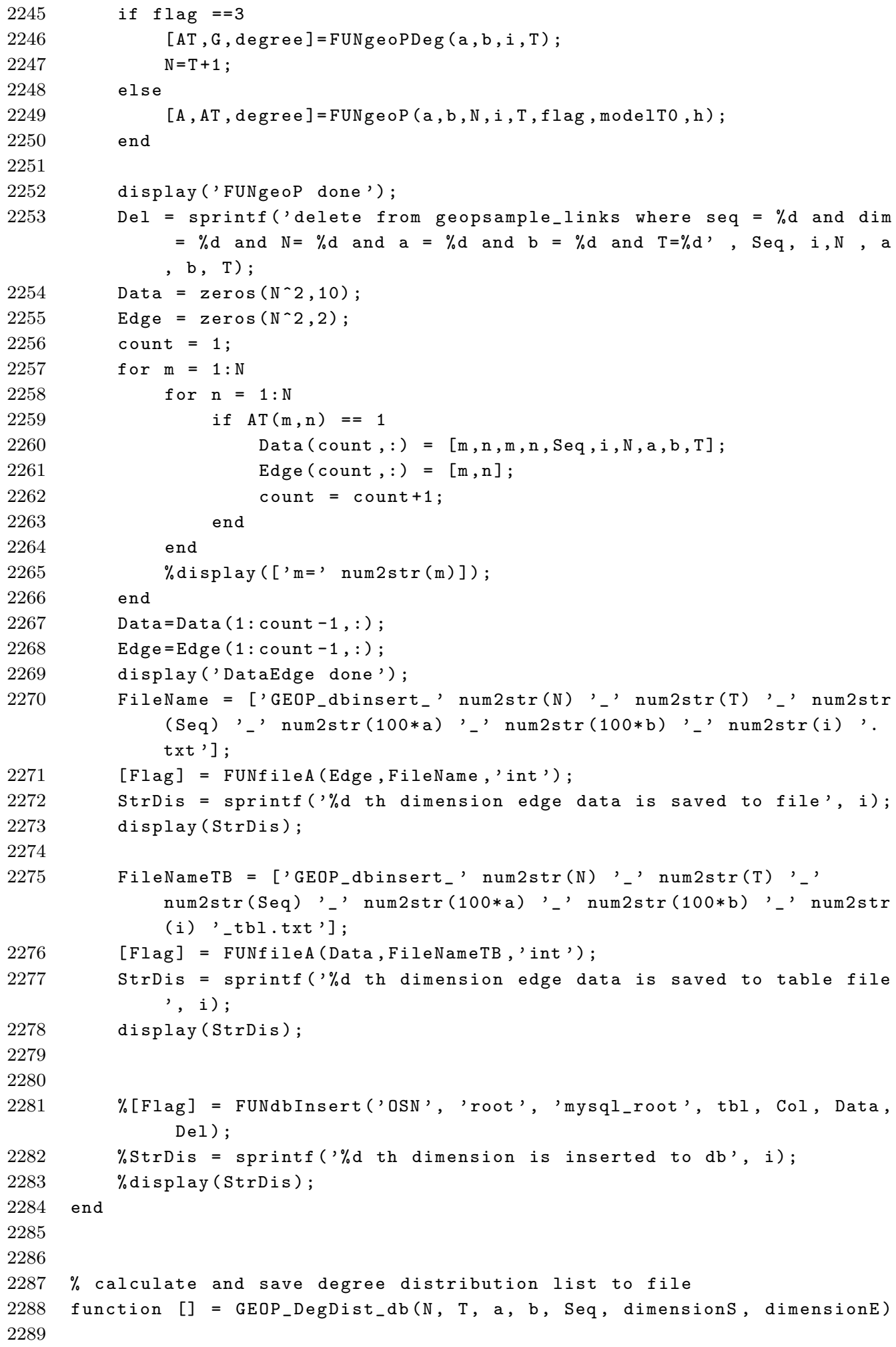




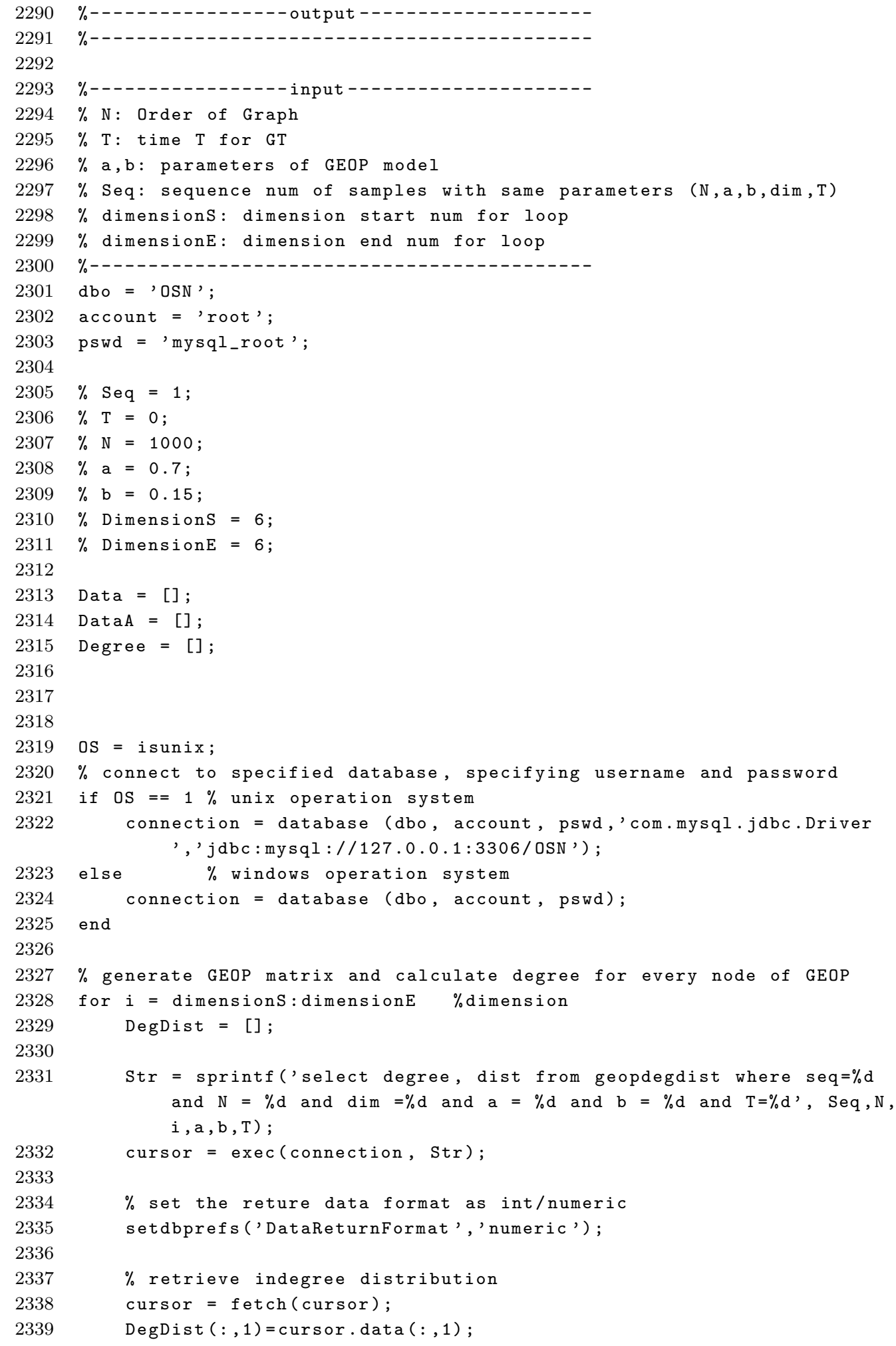




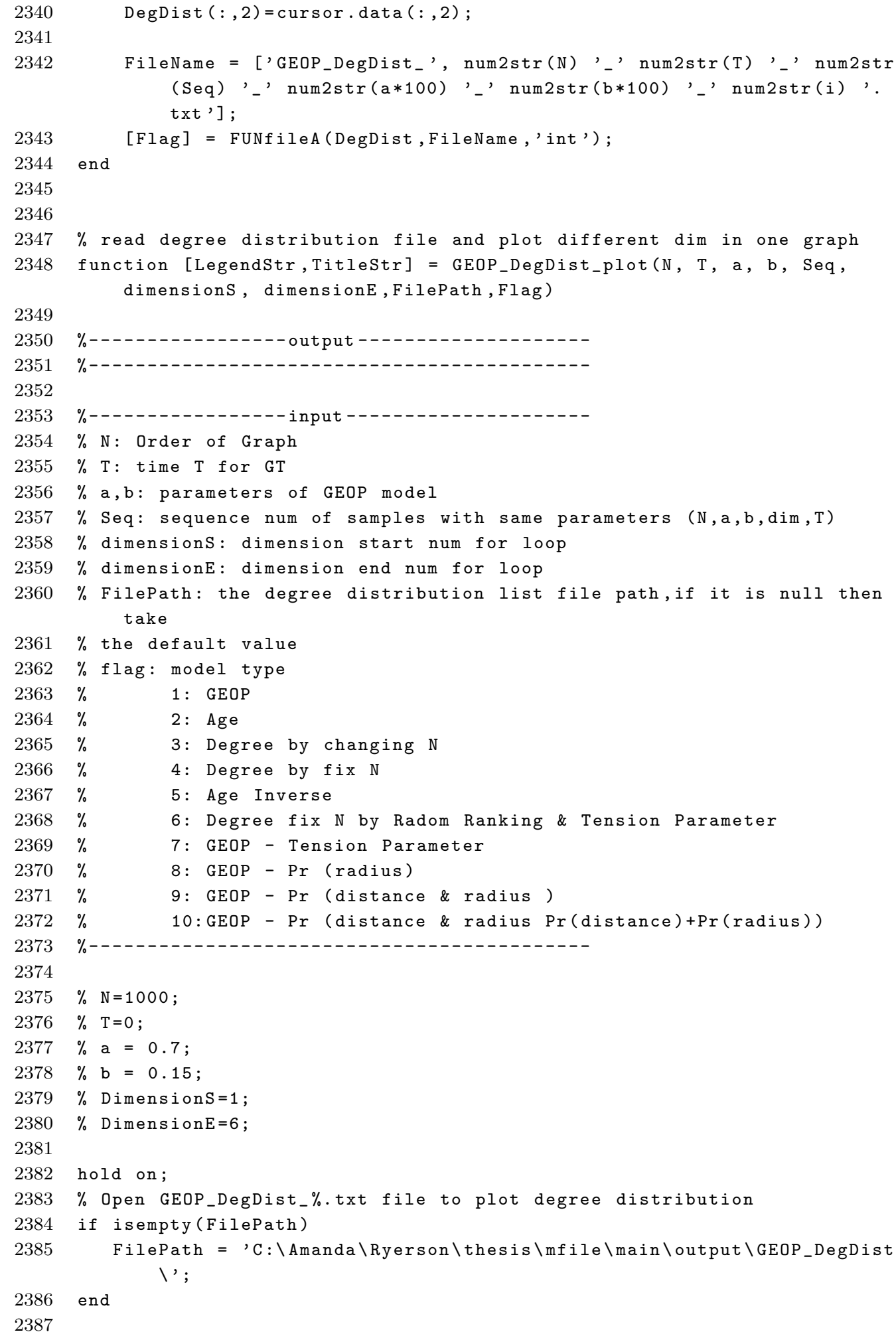




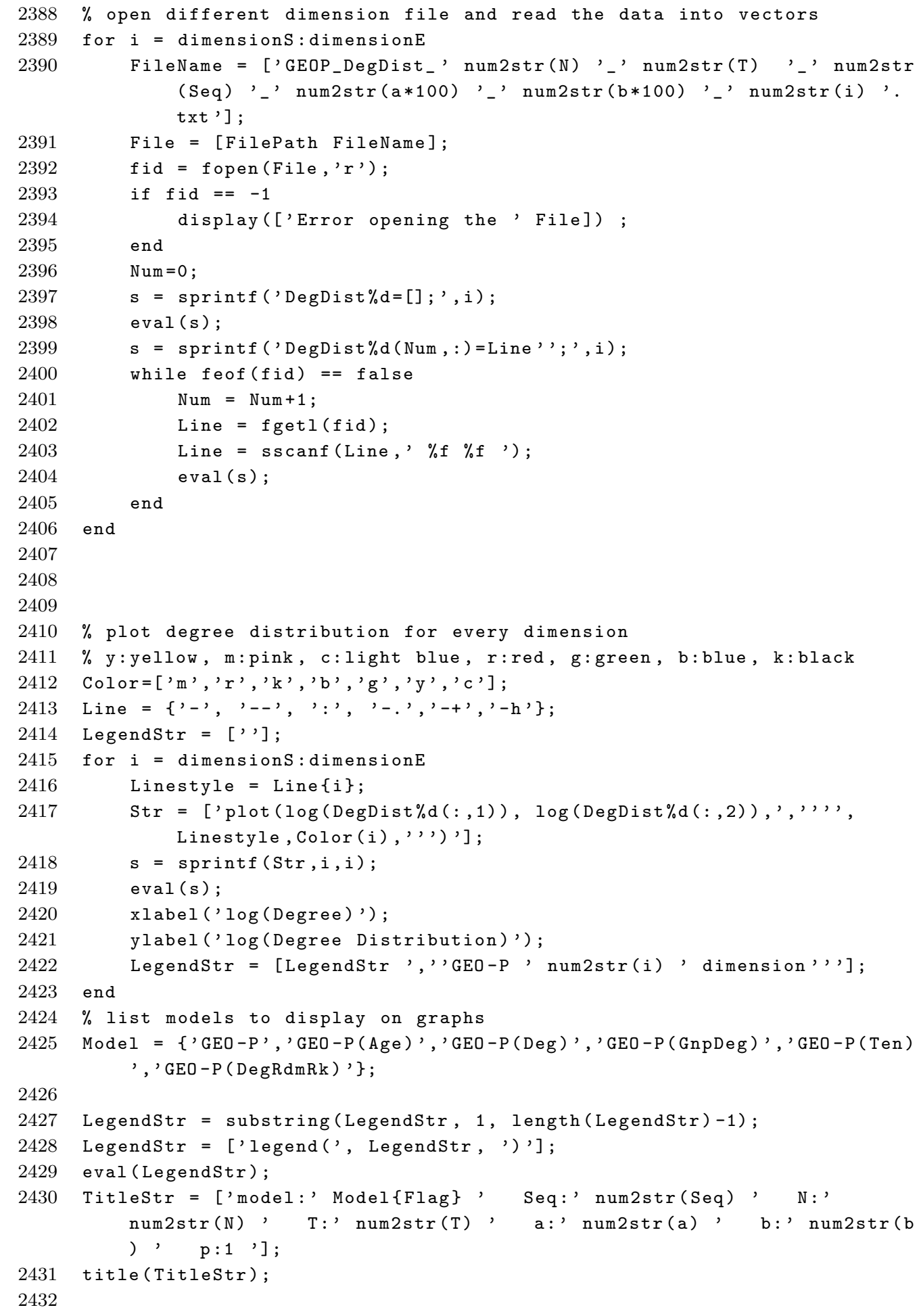




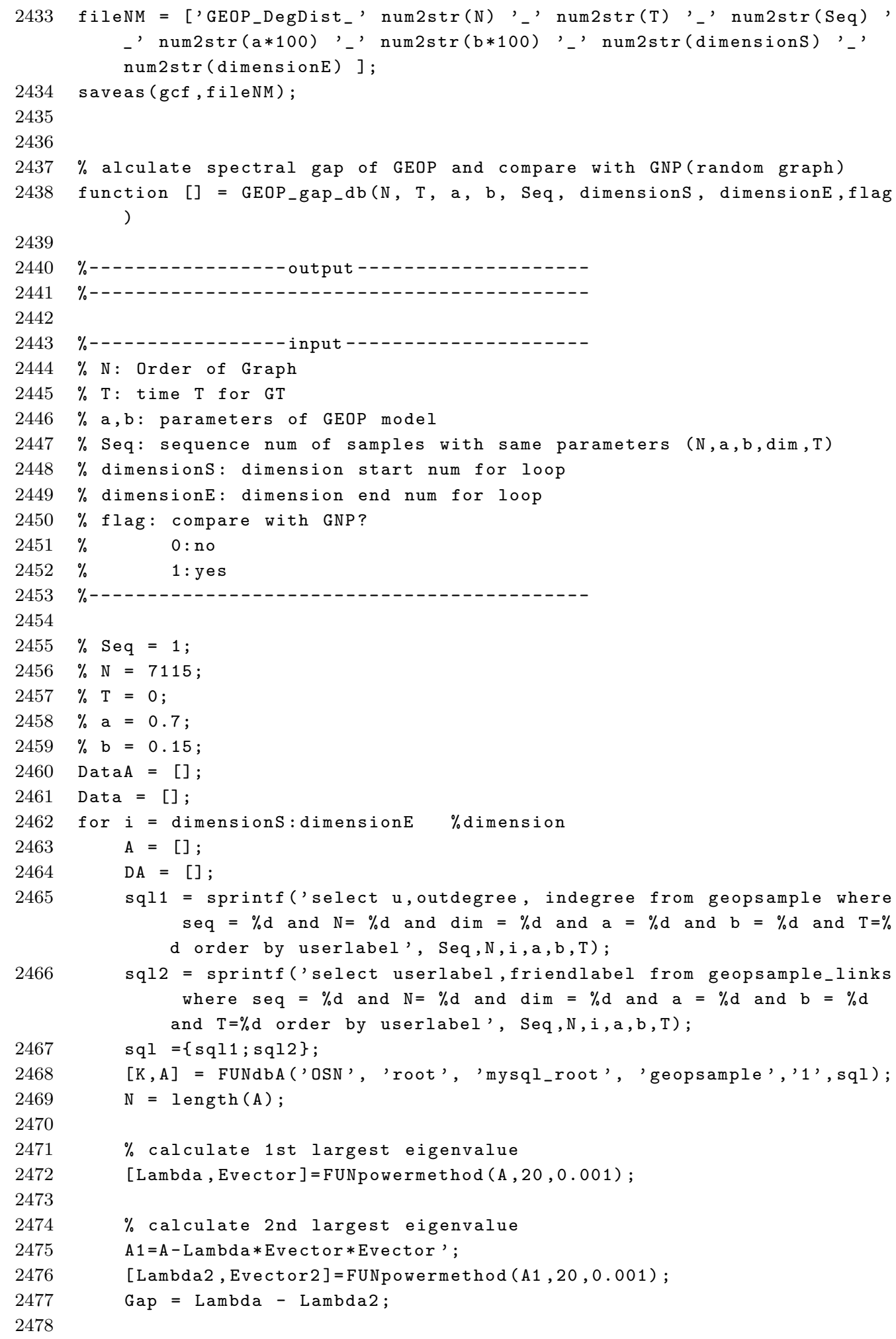




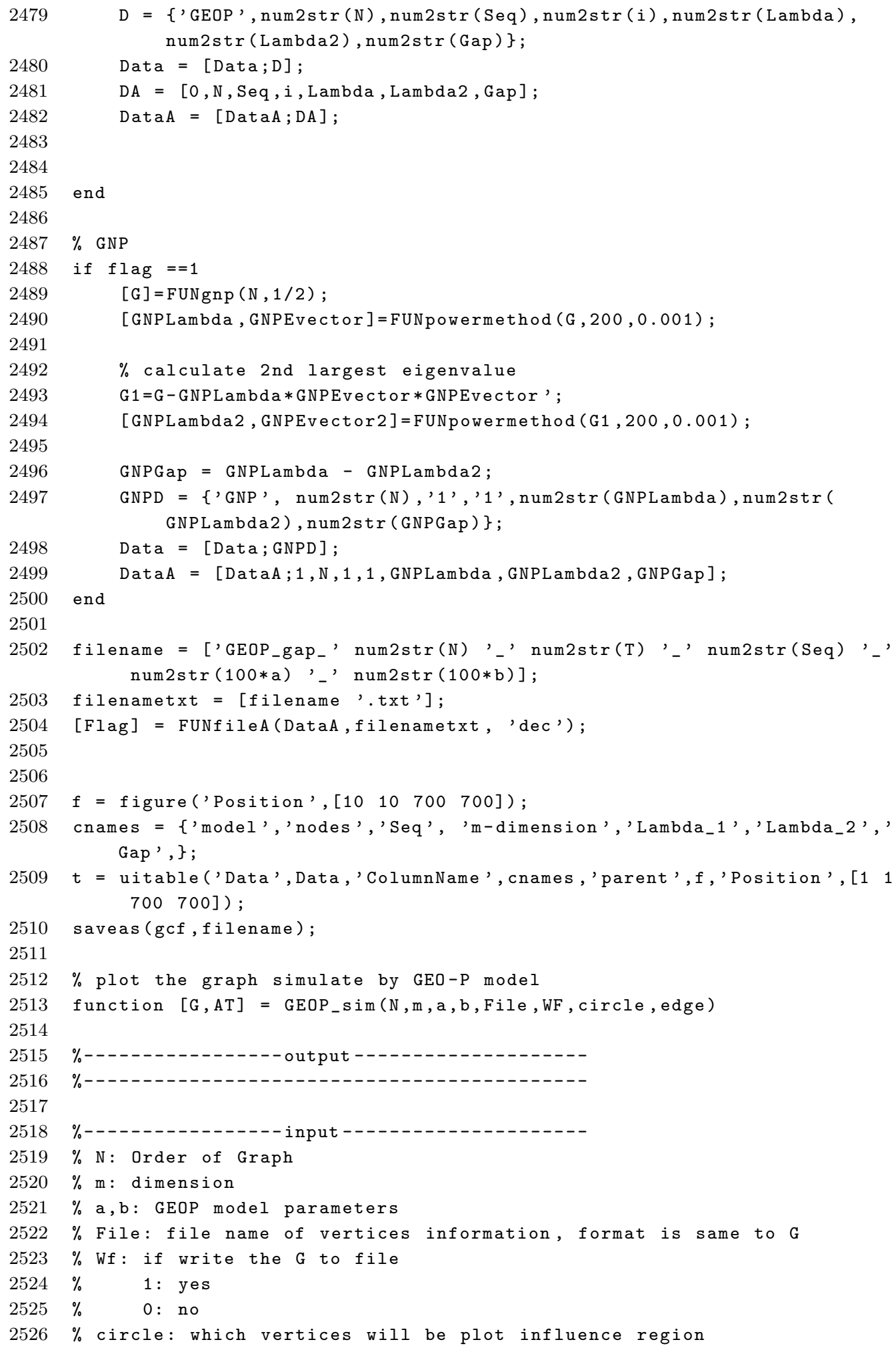




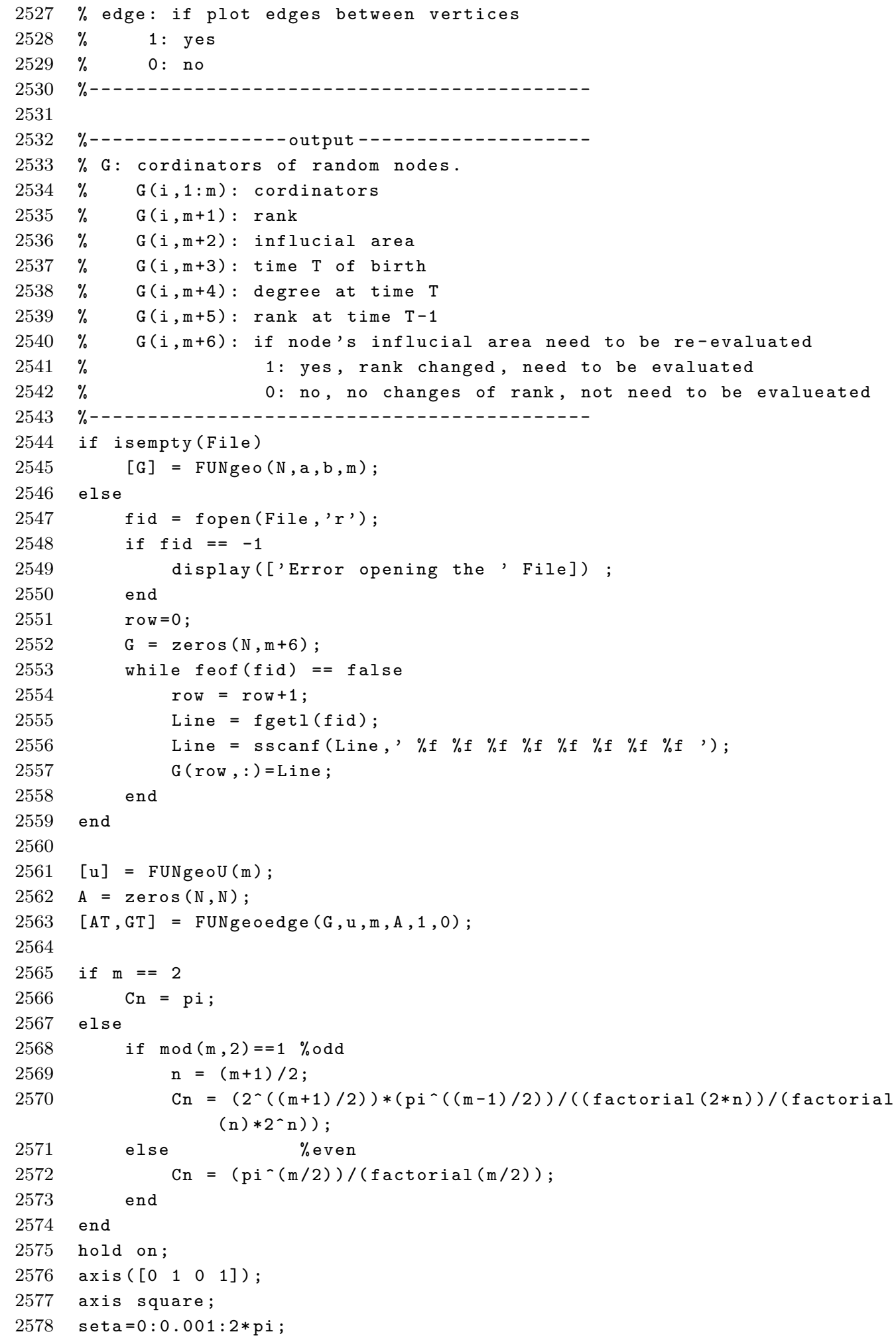




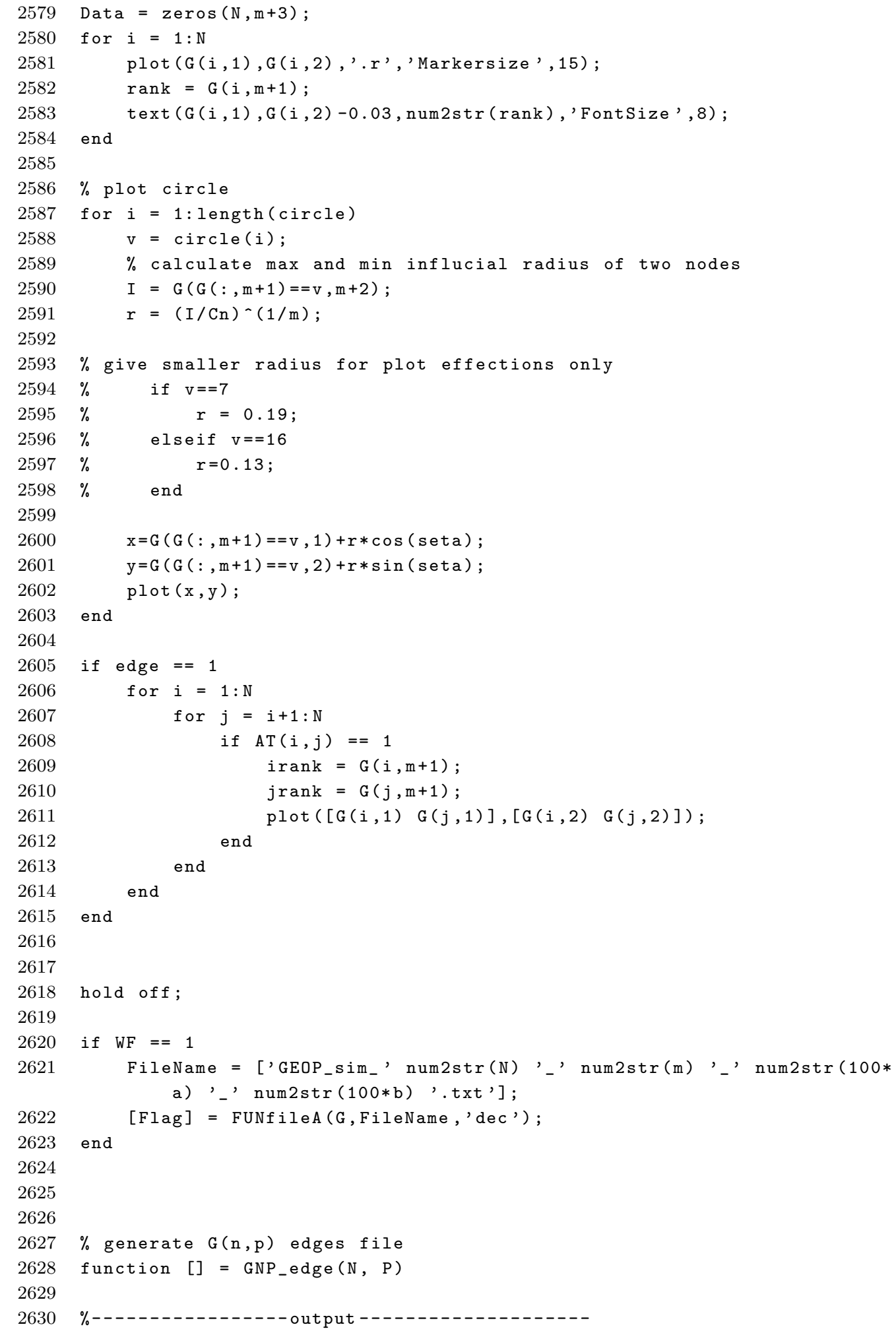




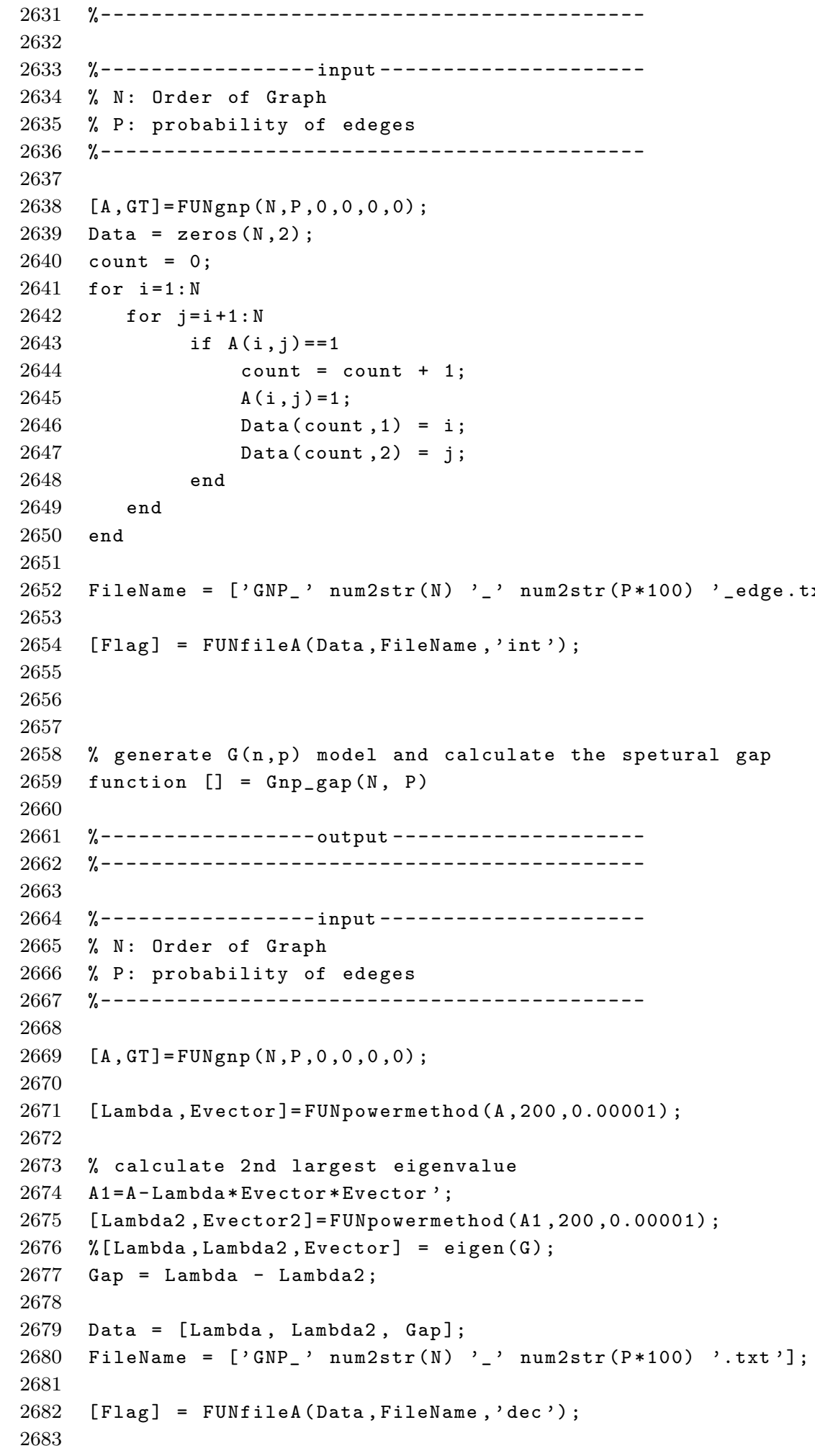




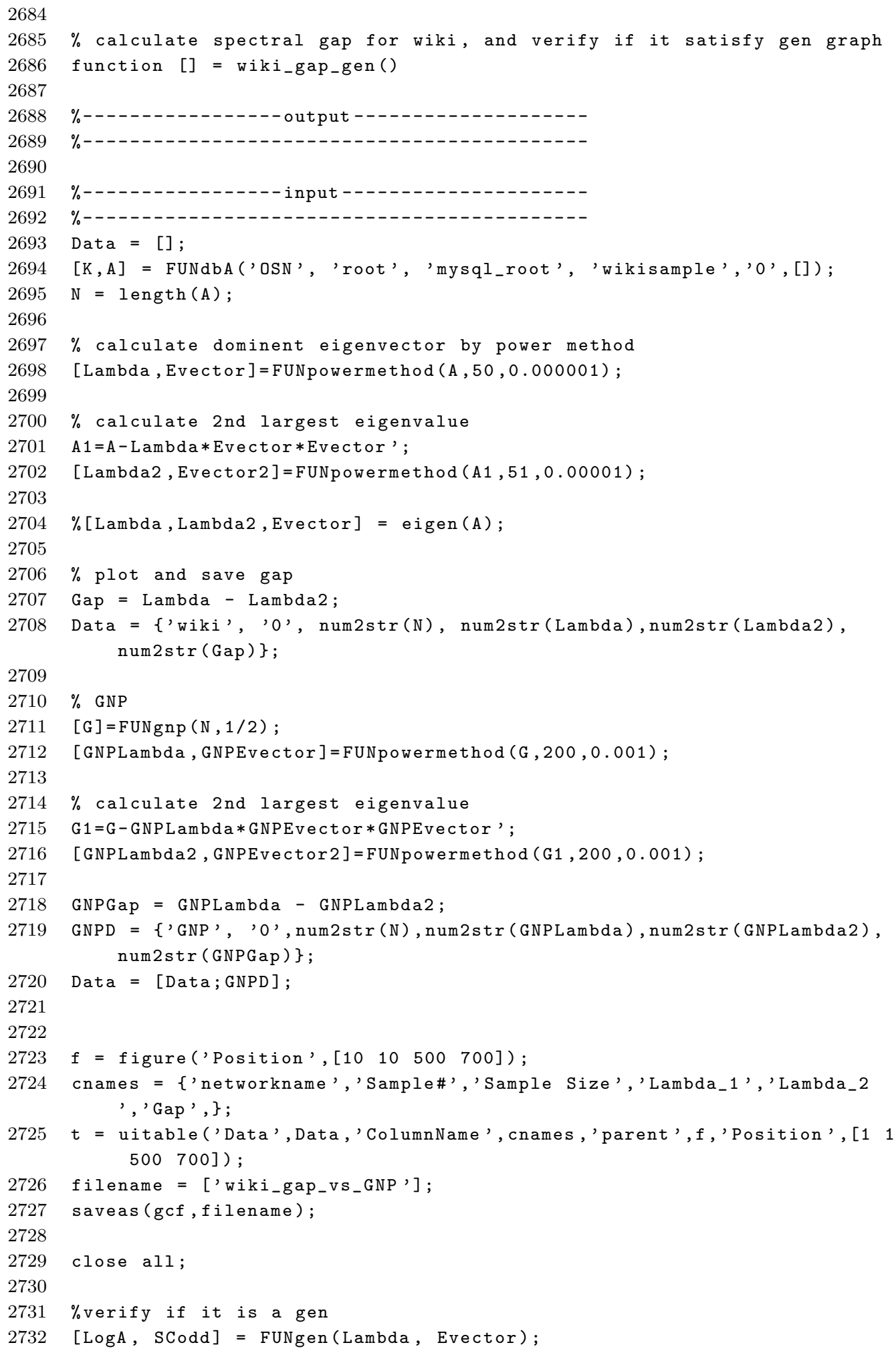




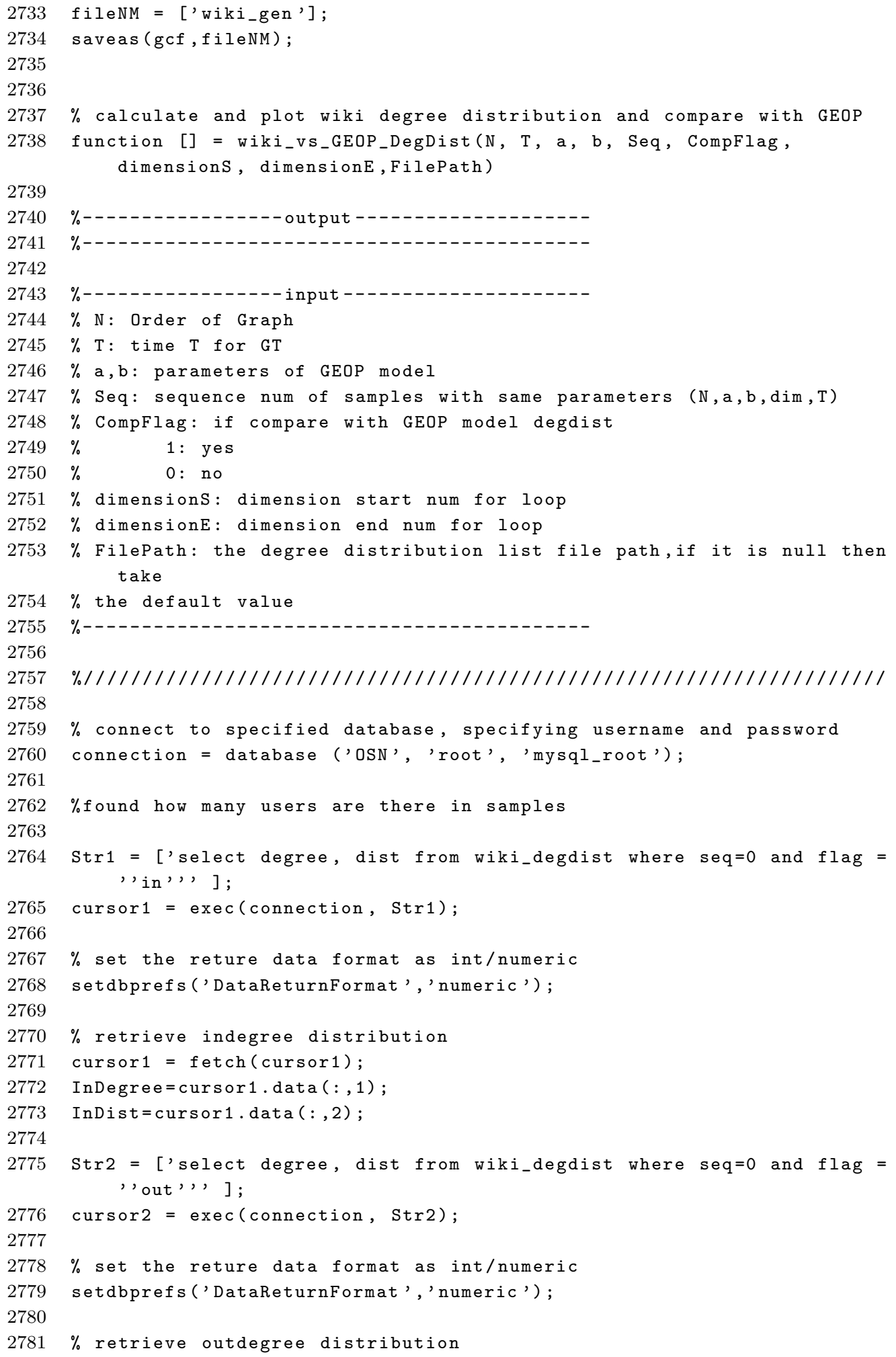




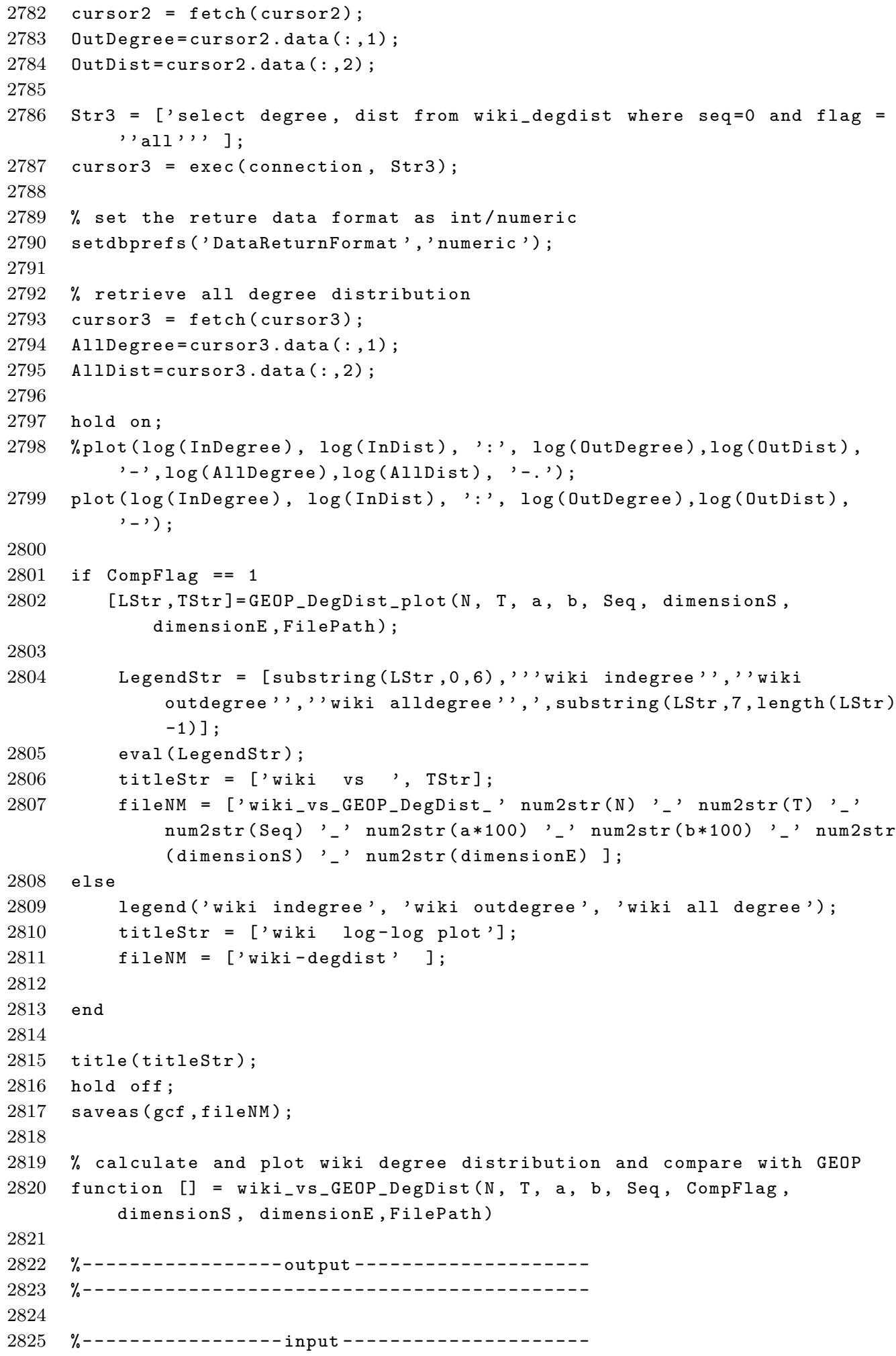




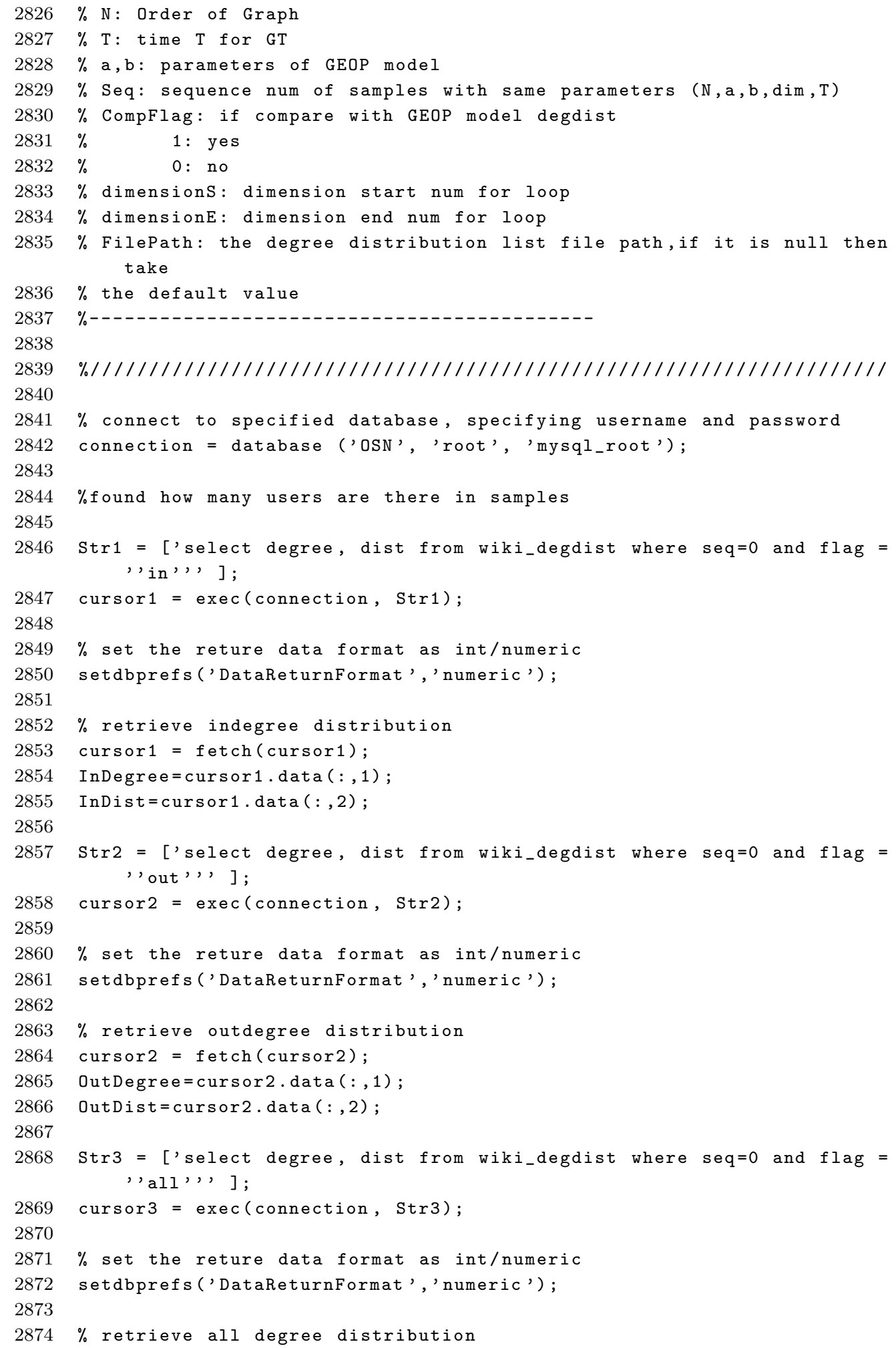




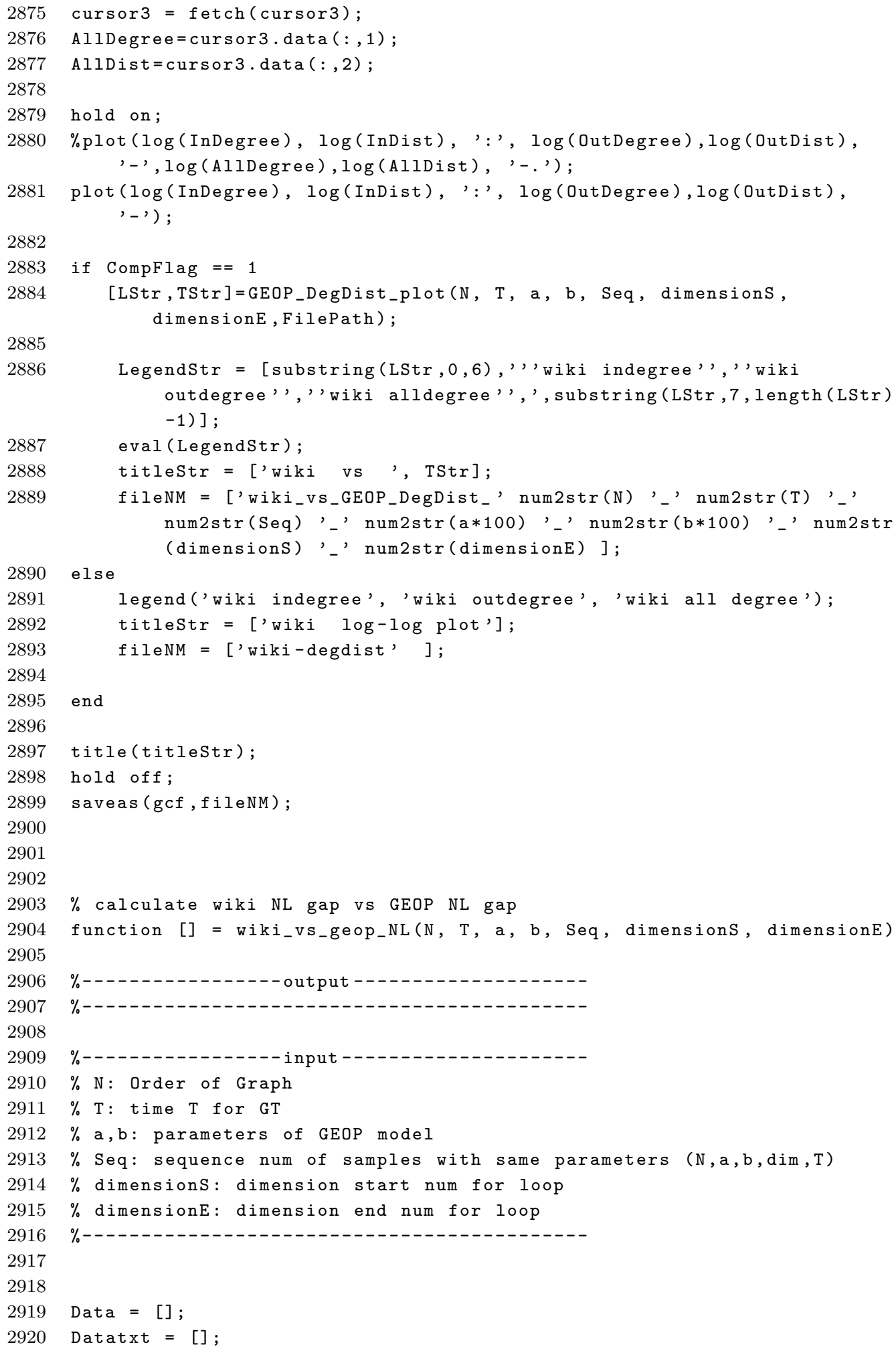




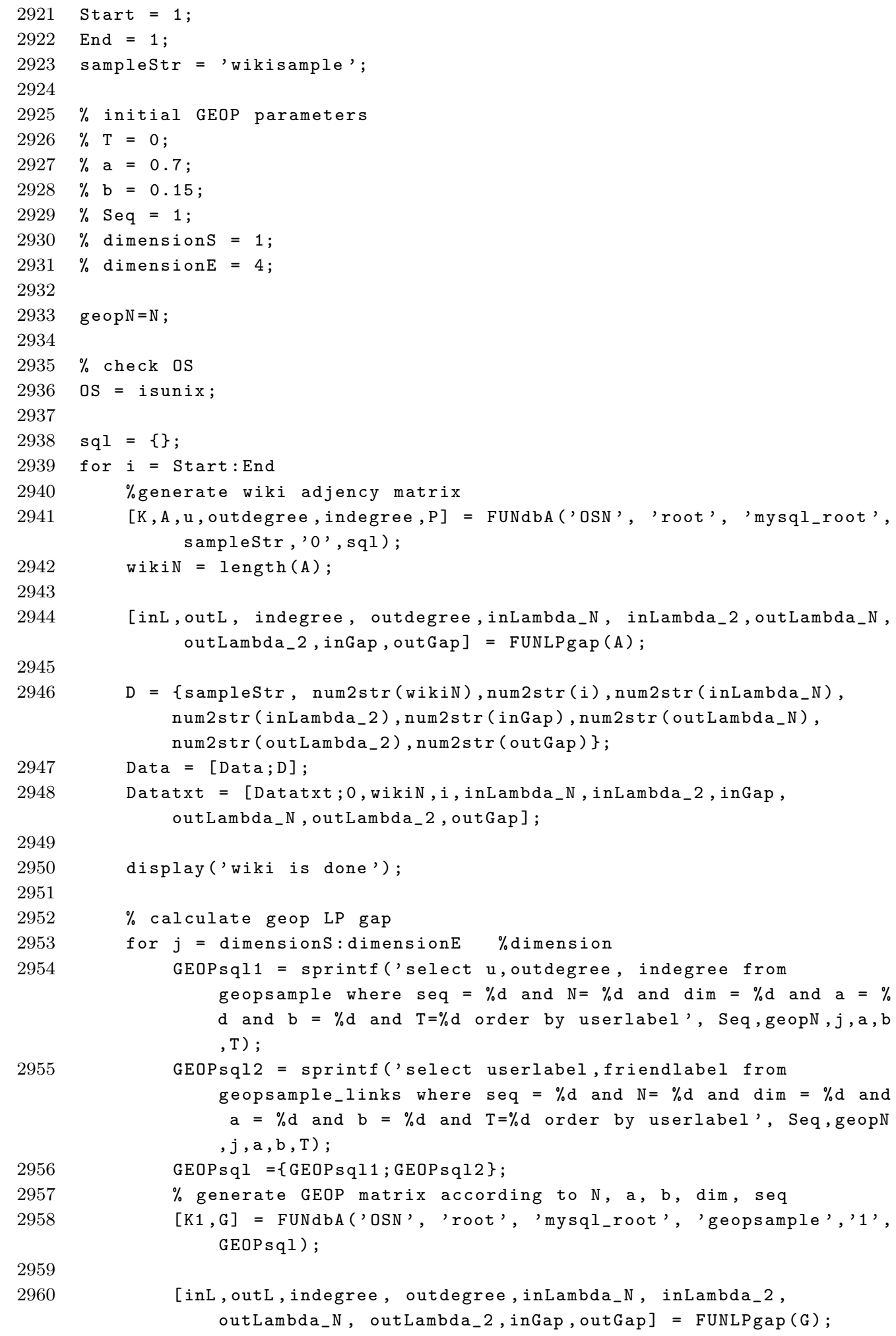




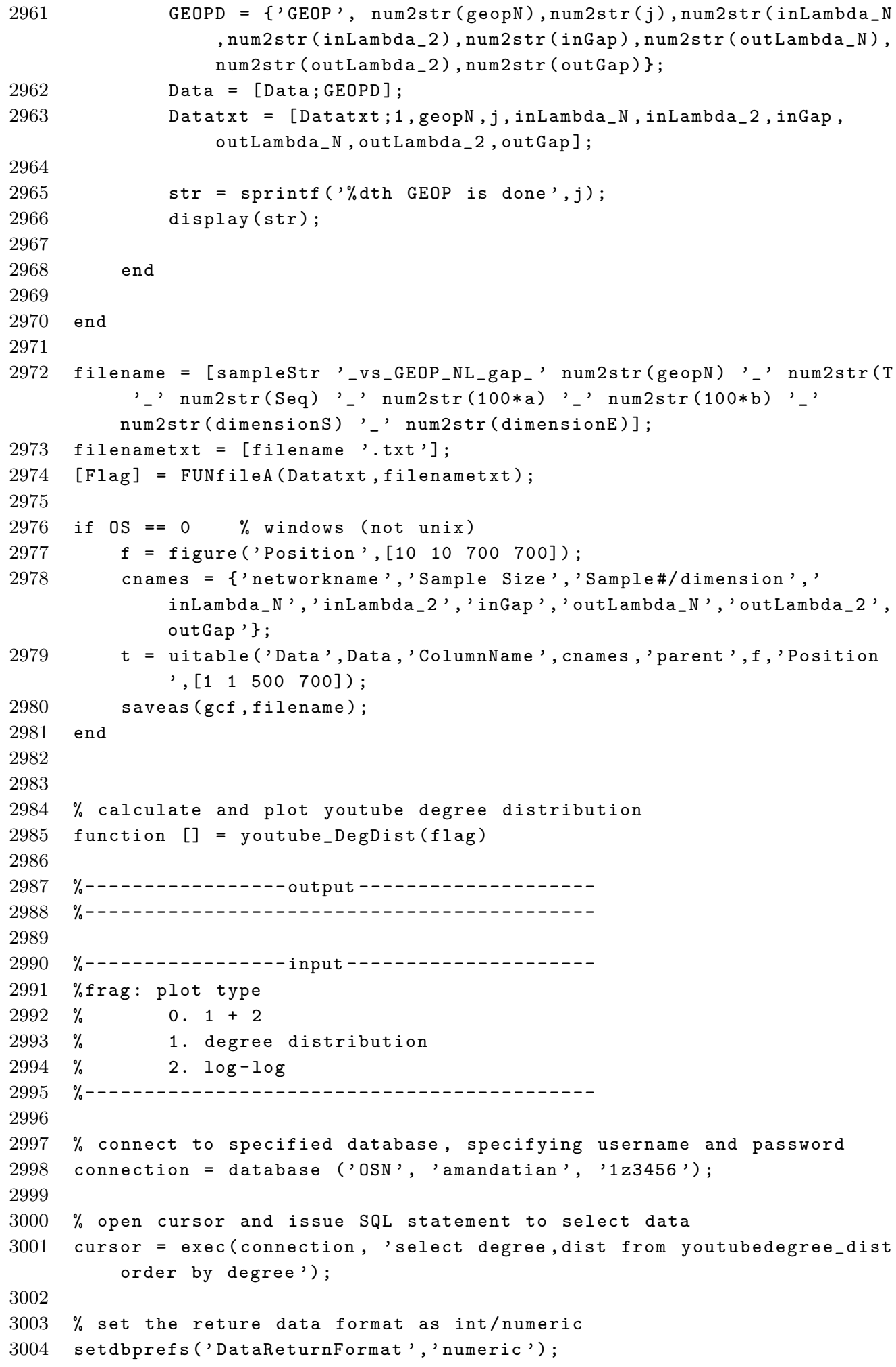

$3003 \%$ set the reture data format as int/numeric 


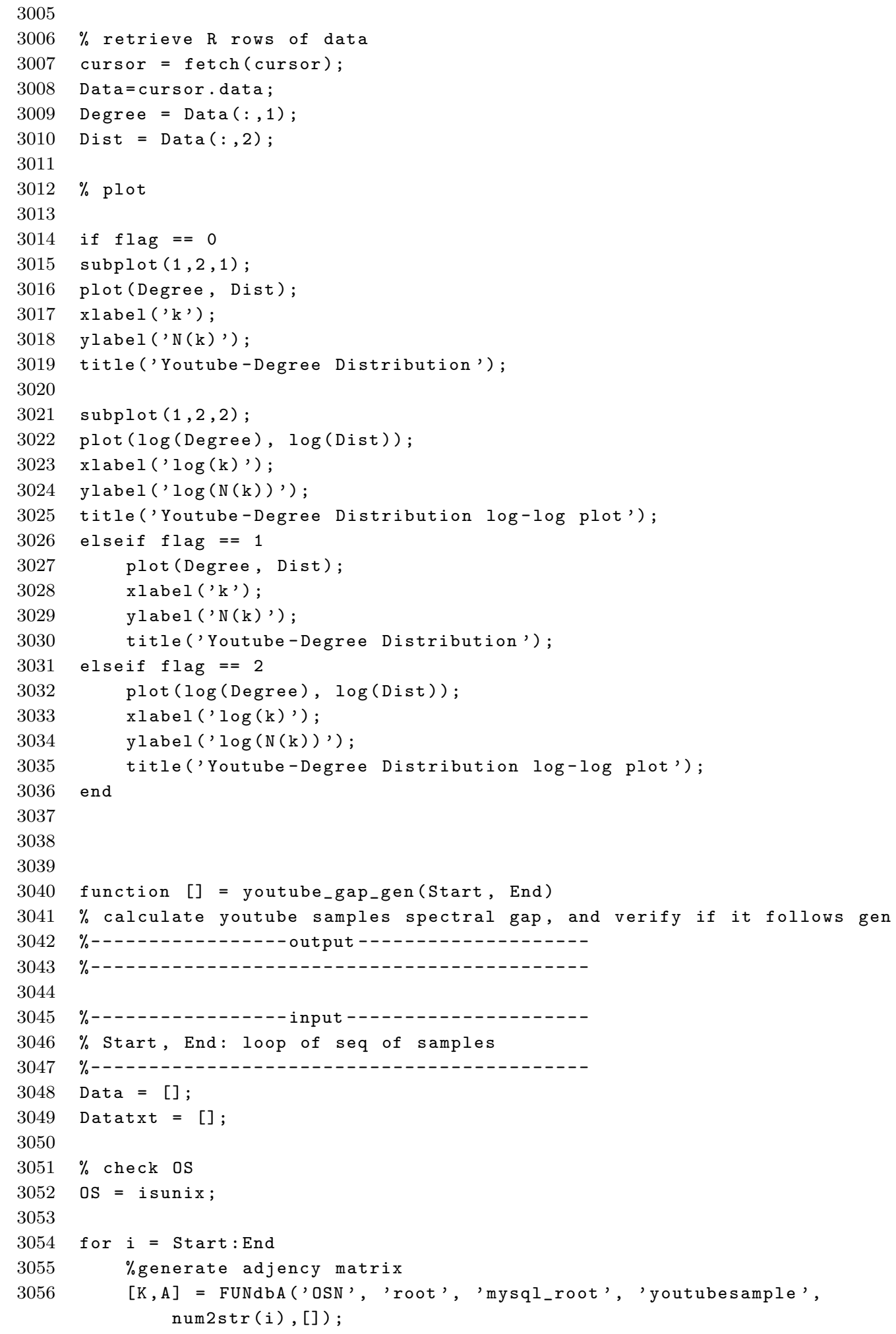




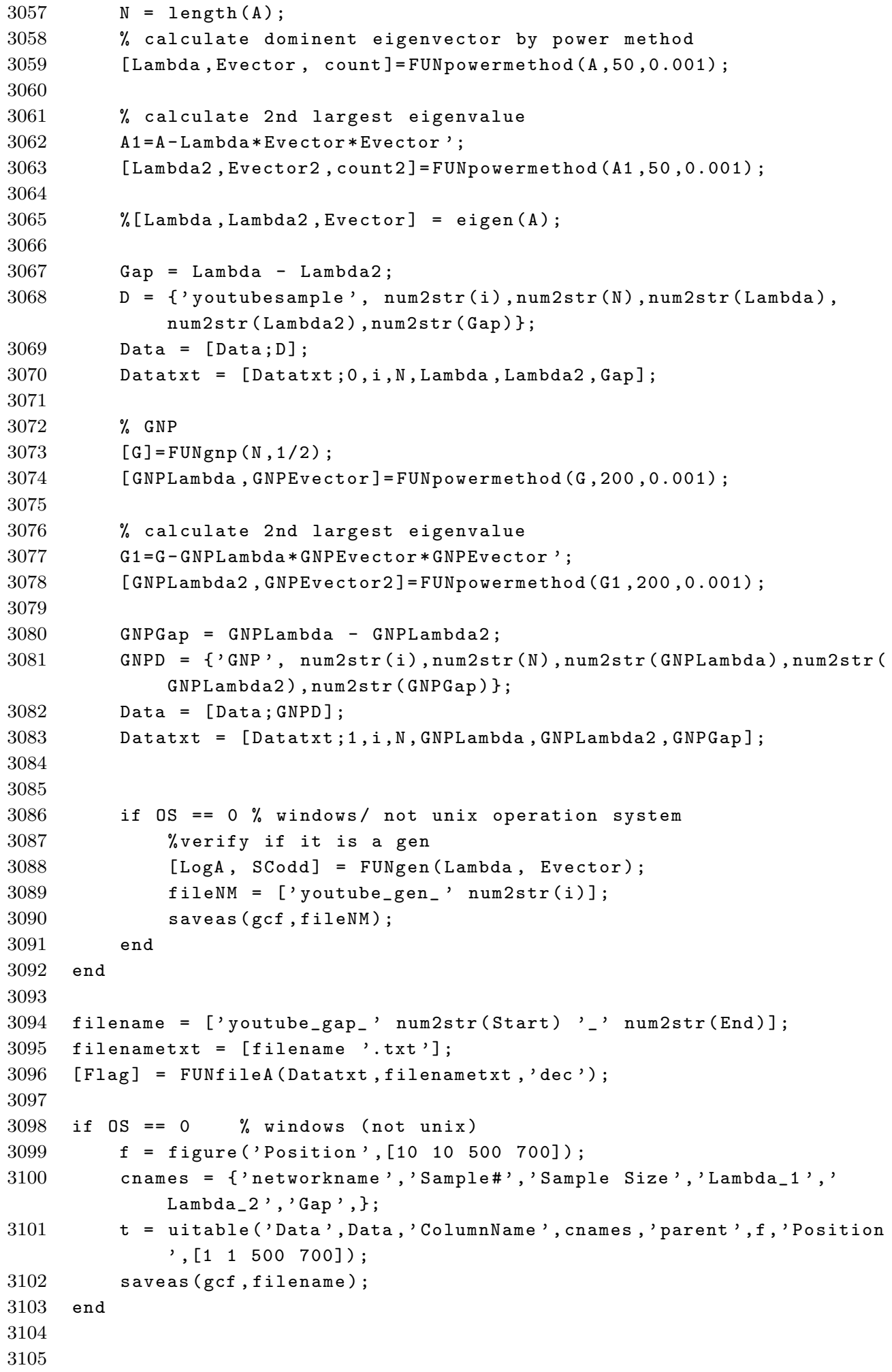

3104

3105 


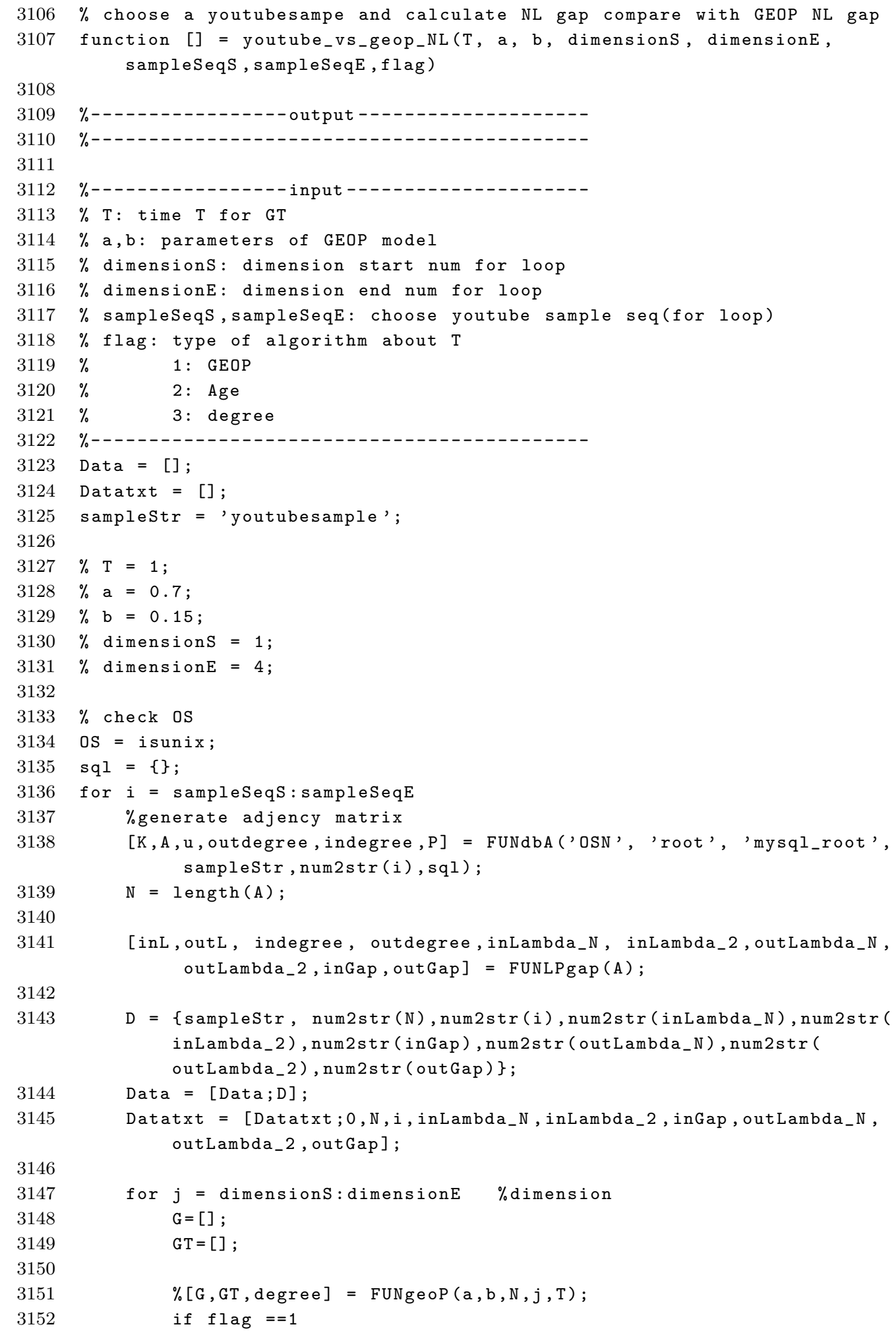

[inL, outL, indegree, outdegree, inLambda_N , inLambda_2, outLambda_N , outLambda_2, inGap, outGap] = FUNLPgap (A); 


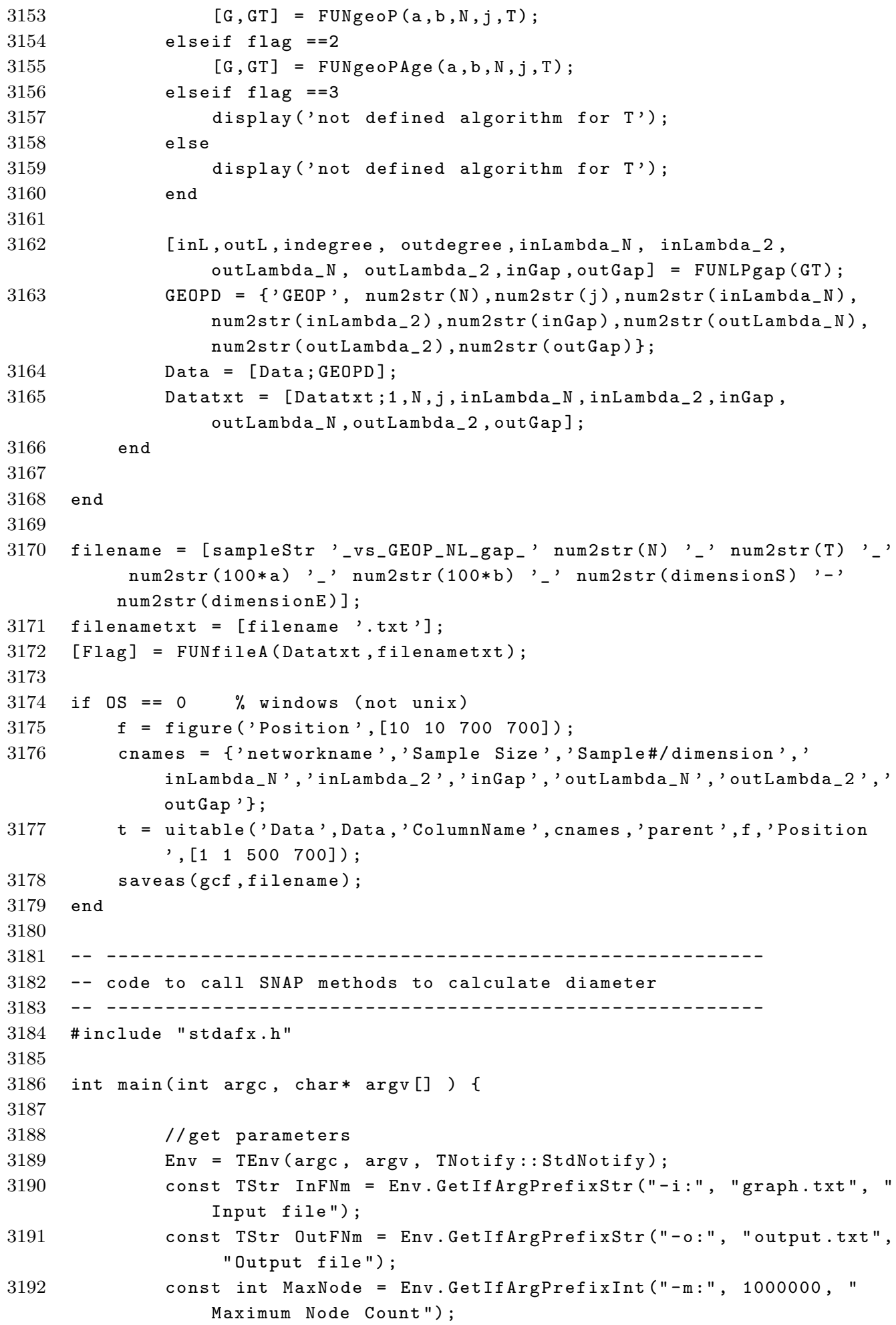

3181

3185

3186

3187

3188

3189

$[\mathrm{G}, \mathrm{GT}]=\mathrm{FUNgeoP}(\mathrm{a}, \mathrm{b}, \mathrm{N}, \mathrm{j}, \mathrm{T}) ;$

elseif flag $==2$

$[\mathrm{G}, \mathrm{GT}]=$ FUNgeoPAge $(\mathrm{a}, \mathrm{b}, \mathrm{N}, \mathrm{j}, \mathrm{T})$;

elseif flag $==3$

display ('not defined algorithm for T') ;

else

display ('not defined algorithm for $T$ ') ;

end

[inL, outL, indegree, outdegree, inLambda_N, inLambda_2 , outLambda_N, outLambda_2, inGap, outGap] = FUNLPgap (GT); $\operatorname{GEOPD}=\left\{{ }^{\prime} \operatorname{GEOP}, \operatorname{num} 2 \operatorname{str}(N), \operatorname{num} 2 \operatorname{str}(j), \operatorname{num} 2 \operatorname{str}(\right.$ inLambda_N$)$, num2str (inLambda_2), num2str (inGap), num2str (outLambda_N), num2str (outLambda_2), num2str (outGap)\};

Data $=[$ Data; GEOPD $]$;

Datatxt $=[$ Datatxt $; 1, N, j$, inLambda_N, inLambda_2, inGap, outLambda_N, outLambda_2, outGap];

end

end

filename $=$ [sampleStr ' _vs_GEOP_NL_gap_, num2str(N) ' , num2str(T) ' num2str(100*a) '_, num2str(100*b) ' _, num2str(dimensions) , '

num2str (dimensionE)];

filenametxt $=[$ filename , t txt'];

$[\mathrm{Flag}]=$ FUNfileA(Datatxt, filenametxt);

if $\mathrm{OS}==0 \quad \%$ windows (not unix)

$f=$ figure('Position', [ [10 10700 700]);

cnames $=$ \{'networkname','Sample Size','Sample\#/dimension', inLambda_N', 'inLambda_2 ', 'inGap', 'outLambda_N', ' outLambda_2 ', ' outGap'\};

$\mathrm{t}=$ uitable('Data', Data, 'ColumnName', cnames, 'parent', f, 'Position , [ [1 1500 700]);

saveas (gcf, filename);

end

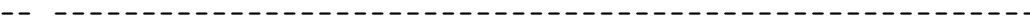

- code to call SNAP methods to calculate diameter

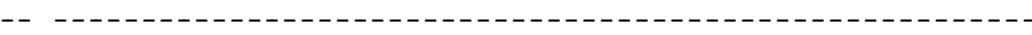

\#include "stdafx.h"

int main(int $\operatorname{argc}, \operatorname{char} * \operatorname{argv}[])\{$

//get parameters

Env $=$ TEnv (argc, argv, TNotify:: StdNotify);

const TStr InFNm = Env.GetIfArgPrefixStr("-i:", "graph.txt", " Input file");

const TStr OutFNm = Env.GetIfArgPrefixStr("-o:", "output.txt", "Output file");

const int MaxNode = Env.GetIfArgPrefixInt ("-m:", 1000000," Maximum Node Count"); 


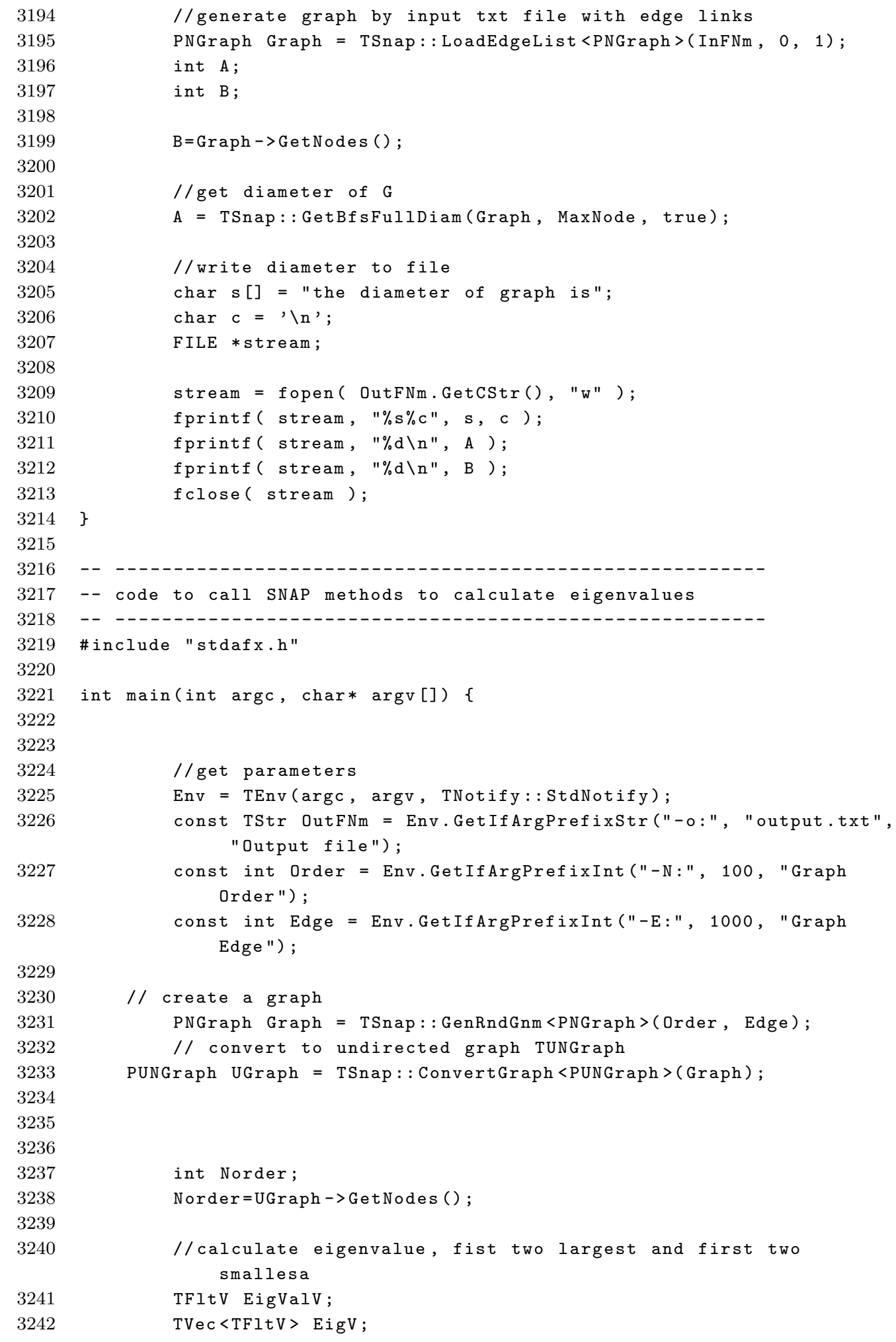




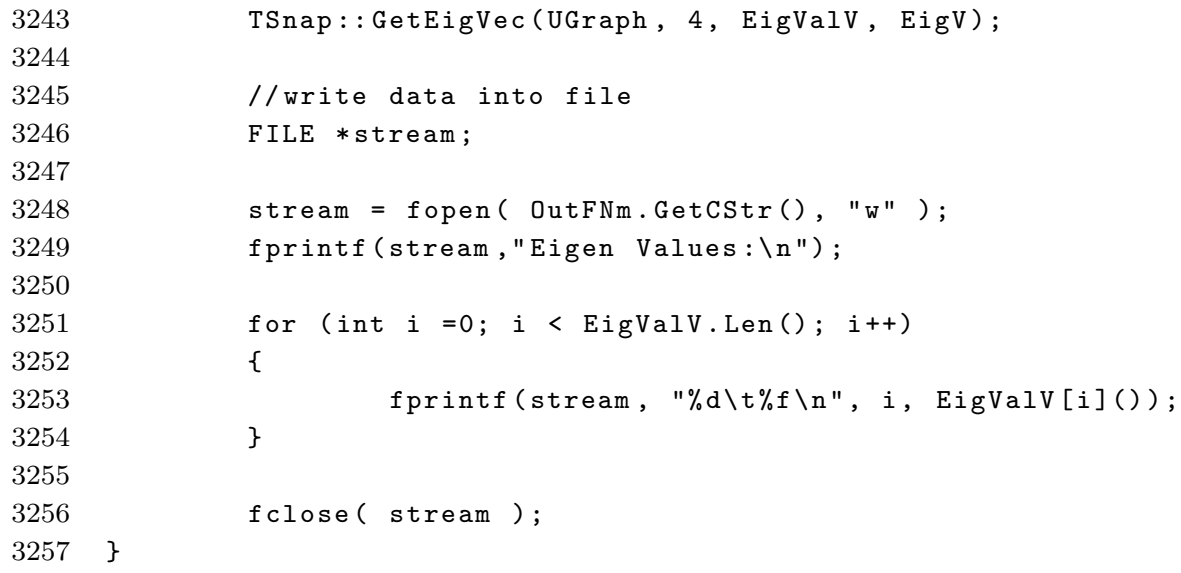





\section{Bibliography}

[1] L.A. Adamic, O. Buyukkokten, E. Adar, A social network caught in the Web, First Monday 8 (2003) 440-442.

[2] Y. Ahn, S. Han, H. Jeong, H. Kwak, S. Moon, Analysis of topological characteristics of huge on-line social networking services, In: Proceedings of the 16th International Conference on World Wide Web, 2007.

[3] A. Bonato, A Course on the Web Graph, American Mathematical Society Graduate Studies Series in Mathematics, Providence, Rhode Island, 2008.

[4] R. Babu, Numerical Methods, Dorling Kindersley (India) Pvt. Ltd., 2010.

[5] A. Barabási, R. Albert, Emergence of scaling in random networks, Science 286 (1999) 509-512.

[6] B. Bhattacharjee, P. Druschel, M. Marcon, A. Mislove, Measurement and analysis of on-line social networks, In: Proceedings of the 7th ACM SIGCOMM Conference on Internet Measurement, 2007.

[7] A. Bonato, J. Janssen, P. Prałat, A geometric model for on-line social networks, In: Proceedings of 3rd Workshop on Online Social Networks, 2010.

[8] F.R.K. Chung, Spectral Graph Theory, American Mathematical Society 1997.

[9] F.R.K. Chung, L. Lu, Complex Graphs and Networks, American Mathematical Society 2004.

[10] E. Estrada, Spectral scaling and good expansion properties in complex networks, Europhys. Lett. 73 (2006) 649-655. 
[11] B. Huberman, S. Golder, D. Wilkinson, Rhythms of social interaction: messaging within a massive on-line network, In: 3rd International Conference on Communities and Technologies, 2007.

[12] R. Kumar, J. Novak, A. Tomkins, Structure and evolution of online social networks, In: Proceedings of the 12th ACM SIGKDD international conference on Knowledge discovery and data mining, 2006.

[13] D. Liben-Nowel, An algorithmic approach to social networks, Ph.D. Dissertation, Massachusetts Institute of Technology, 2005.

[14] List of social networking websites, Wikipedia. Accessed July 1, 2011. http: //en.wikipedia.org/wiki/List_of_social_networking_websites.

[15] S. Milgram, The small world problem, Psychology Today 2 (1967) 60-67.

[16] Online Social Network Research@The Max Planck Institute for Software Systems. Accessed May 10, 2010. http://socialnetworks.mpi-sws.org/ data-imc2007.html

[17] Press Room, Facebook. Accessed July 1, 2011. http://www.facebook.com/ press/info.php?statistics.

[18] Stanford large network dataset collection. Accessed July 5, 2010. http: //snap.stanford.edu/data/index.html

[19] The History of Usenet, Usenet.com. Accessed July 1, 2011. http://www. usenet.com/usenet_history.html

[20] Usenet, Wikipedia. Accessed July 1, 2011. http://en.wikipedia.org/wiki/ Usenet.

[21] D. J. Watts, S. H. Strogatz, Collective dynamics of "small-world" networks, Nature 393 (1998) 440-442. 